\title{
Bulky Diphosphine Acetate Ruthenium Complexes: Synthesis and Catalytic Activity in Ketone Transfer Hydrogenation and Alkyne Dimerization
}

\author{
Maurizio Ballico, Daniele Zuccaccia, Rosario Figliolia,* Walter Baratta*
}

Dipartimento DI4A - Università di Udine, Via del Cotonificio 108, I-33100 Udine, Italy

E-mails: walter.baratta@uniud.it, figliolia.rosario@spes.uniud.it

\section{Supplementary Information}

Table of Contents:

Figure S1. ${ }^{31} \mathrm{P}\left\{{ }^{1} \mathrm{H}\right\}$ NMR spectrum of $\left[\mathrm{Ru}\left(\eta^{2}-\mathrm{OAc}\right)_{2}(\mathrm{DCyPF})\right](\mathbf{1 - c y})$

Pag. S6

Figure S2. ${ }^{1} \mathrm{H}$ NMR spectrum of $\left[\mathrm{Ru}\left(\eta^{2}-\mathrm{OAc}\right)_{2}(\mathrm{DCyPF})\right](\mathbf{1}-\boldsymbol{c y})$

Pag. S7

Figure S3. ${ }^{31} \mathrm{P}\left\{{ }^{1} \mathrm{H}\right\}$ NMR spectrum of trans-[Ru( $\left.\left.\eta^{1}-\mathrm{OAc}\right)_{2}(\mathrm{D} i \mathrm{PPF})(\mathrm{en})\right](\mathbf{2})$

Pag. S8

Figure S4. ${ }^{1} \mathrm{H}$ NMR spectrum of trans-[Ru( $\left.\left.\eta^{1}-\mathrm{OAc}\right)_{2}(\mathrm{DiPPF})(\mathrm{en})\right](\mathbf{2})$

Pag. S9

Figure S5. ${ }^{13} \mathrm{C}\left\{{ }^{1} \mathrm{H}\right\}$ NMR spectrum of trans-[Ru( $\left.\left.\eta^{1}-\mathrm{OAc}\right)_{2}(\mathrm{D} i \mathrm{PPF})(\mathrm{en})\right]$

Pag. S10

Figure S6. ${ }^{31} \mathrm{P}\left\{{ }^{1} \mathrm{H}\right\}$ NMR spectrum of trans $-\left[\mathrm{Ru}\left(\eta^{1}-\mathrm{OAc}\right)_{2}(\mathrm{DCyPF})(\mathrm{en})\right](\mathbf{3})$

Pag. S11

Figure S7. ${ }^{1} \mathrm{H}$ NMR spectrum of trans- $\left[\mathrm{Ru}\left(\eta^{1}-\mathrm{OAc}\right)_{2}(\mathrm{DCyPF})(\mathrm{en})\right](\mathbf{3})$

Pag. S12

Figure S8. ${ }^{13} \mathrm{C}\left\{{ }^{1} \mathrm{H}\right\}$ PENDANT NMR spectrum of trans $-\left[\mathrm{Ru}\left(\eta^{1}-\mathrm{OAc}\right)_{2}(\mathrm{DCyPF})(\mathrm{en})\right](3)$

Pag. S13

Figure S9. ${ }^{31} \mathrm{P}\left\{{ }^{1} \mathrm{H}\right\}$ NMR spectrum of trans-[Ru( $\left.\left.\eta^{1}-\mathrm{OAc}\right)_{2}(\mathrm{DiPPF})(\operatorname{ampy})\right](4)$ in $\mathrm{CD}_{2} \mathrm{Cl}_{2}$ at $-60{ }^{\circ} \mathrm{C}$

Pag. S14

Figure S10. ${ }^{1} \mathrm{H}$ NMR spectrum of trans $-\left[\mathrm{Ru}\left(\eta^{1}-\mathrm{OAc}\right)_{2}(\mathrm{D} i \mathrm{PPF})(\mathrm{ampy})\right](4)$ in $\mathrm{CD}_{2} \mathrm{Cl}_{2}$ at $-60{ }^{\circ} \mathrm{C}$ 
Figure S11. ${ }^{13} \mathrm{C}\left\{{ }^{1} \mathrm{H}\right\}$ NMR spectrum of trans-[Ru( $\left.\left.\eta^{1}-\mathrm{OAc}\right)_{2}(\mathrm{D} i \mathrm{PPF})(\operatorname{ampy})\right](4)$ in $\mathrm{CD}_{2} \mathrm{Cl}_{2}$ at -60 ${ }^{\circ} \mathrm{C}$

Figure S12. ${ }^{31} \mathrm{P}\left\{{ }^{1} \mathrm{H}\right\}$ NMR spectrum of trans-[Ru( $\left.\left.\eta^{1}-\mathrm{OAc}\right)_{2}(\mathrm{D} i \mathrm{PPF})(\operatorname{ampy})\right](4)$ in $\mathrm{CD}_{2} \mathrm{Cl}_{2}$ at $0{ }^{\circ} \mathrm{C}$

Figure S13. ${ }^{1} \mathrm{H}$ NMR spectrum of trans-[Ru( $\left.\left.\eta^{1}-\mathrm{OAc}\right)_{2}(\mathrm{DiPPF})(\mathrm{ampy})\right](4)$ in $\mathrm{CD}_{2} \mathrm{Cl}_{2}$ at $0{ }^{\circ} \mathrm{C}$

Pag. S18

Figure S14. Effect of the heating on the solution of trans-[Ru( $\left.\left.\eta^{1}-\mathrm{OAc}\right)_{2}(\mathrm{D} i \mathrm{PPF})(\operatorname{ampy})\right](4)$ in $\mathrm{CD}_{2} \mathrm{Cl}_{2}$ from $0{ }^{\circ} \mathrm{C}$ to $\mathrm{RT}:{ }^{31} \mathrm{P}\left\{{ }^{1} \mathrm{H}\right\}$ NMR spectrum after $24 \mathrm{~h}$ Pag. S19

Figure S15. Stacked VT ${ }^{31} \mathrm{P}\left\{{ }^{1} \mathrm{H}\right\}$ NMR spectra from $0{ }^{\circ} \mathrm{C}$ to $\mathrm{RT}$ of the solution of trans-[Ru$\left(\eta^{1}-\right.$ $\left.\mathrm{OAc})_{2}(\mathrm{DiPPF})(\mathrm{ampy})\right](4)$ in $\mathrm{CD}_{2} \mathrm{Cl}_{2}$

Pag. S20

Figure S16. Stacked VT ${ }^{31} \mathrm{P}\left\{{ }^{1} \mathrm{H}\right\}$ NMR spectra from RT to $75^{\circ} \mathrm{C}$ of the products of the reaction between $\left[\mathrm{Ru}\left(\eta^{1}-\mathrm{OAc}\right)\left(\eta^{2}-\mathrm{OAc}\right)(\mathrm{D} i \mathrm{PPF})\right](\mathbf{1}-\mathbf{i} \boldsymbol{p})$ and ampy (3 equiv) in isopropanol- $d^{8} \quad$ Pag. S21

Figure S17. Stacked VT ${ }^{31} \mathrm{P}\left\{{ }^{1} \mathrm{H}\right\}$ NMR spectra from RT to $50{ }^{\circ} \mathrm{C}$ of the products of the reaction between $\left[\mathrm{Ru}\left(\eta^{1}-\mathrm{OAc}\right)\left(\eta^{2}-\mathrm{OAc}\right)(\mathrm{D} i \mathrm{PPF})\right](\mathbf{1}-\mathbf{i} \boldsymbol{p})$ and ampy $\left(1.3\right.$ equiv) in toluene- $d^{8} \quad$ Pag. S21

Figure S18. ${ }^{31} \mathrm{P}\left\{{ }^{1} \mathrm{H}\right\}$ NMR spectrum of the mixture $4: 1$ of the cationic species $\left[\mathrm{Ru}\left(\eta^{2}-\right.\right.$ $\mathrm{OAc})(\mathrm{D} i \mathrm{PPF})(\mathrm{ampy})] \mathrm{OAc}(\mathbf{5 a})$ and $c i s-\left[\mathrm{Ru}\left(\eta^{1}-\mathrm{OAc}\right)_{2}(\mathrm{D} i \mathrm{PPF})(\mathrm{ampy})\right](\mathbf{5 b})$ in $\mathrm{CD}_{2} \mathrm{Cl}_{2} / \mathrm{CD}_{3} \mathrm{OD}$

Pag. S22

Figure S19. ${ }^{1} \mathrm{H}$ NMR spectrum of the mixture $4: 1$ of the cationic species $\left[\mathrm{Ru}\left(\eta^{2}-\right.\right.$ $\mathrm{OAc})(\mathrm{D} i \mathrm{PPF})(\mathrm{ampy})] \mathrm{OAc}(\mathbf{5 a})$ and $c i s-\left[\mathrm{Ru}\left(\eta^{1}-\mathrm{OAc}\right)_{2}(\mathrm{D} i \mathrm{PPF})(\mathrm{ampy})\right](\mathbf{5 b})$ in $\mathrm{CD}_{2} \mathrm{Cl}_{2} / \mathrm{CD}_{3} \mathrm{OD}$

Pag. S23

Figure S20. ${ }^{13} \mathrm{C}\left\{{ }^{1} \mathrm{H}\right\}$ PENDANT NMR spectrum of the mixture 4:1 of the cationic species $\left[\mathrm{Ru}\left(\eta^{2}-\right.\right.$ $\mathrm{OAc})(\mathrm{D} i \mathrm{PPF})(\mathrm{ampy})] \mathrm{OAc}(\mathbf{5 a})$ and $c i s-\left[\mathrm{Ru}\left(\eta^{1}-\mathrm{OAc}\right)_{2}(\mathrm{D} i \mathrm{PPF})(\mathrm{ampy})\right](\mathbf{5 b})$ in $\mathrm{CD}_{2} \mathrm{Cl}_{2} / \mathrm{CD}_{3} \mathrm{OD}$ 
Figure S21. ${ }^{31} \mathrm{P}\left\{{ }^{1} \mathrm{H}\right\}$ NMR spectrum of the mixture $4: 1$ of the cationic species $\left[\mathrm{Ru}\left(\eta^{2}-\right.\right.$ $\mathrm{OAc})(\mathrm{D} i \mathrm{PPF})(\mathrm{ampy})] \mathrm{OAc}(\mathbf{5 a})$ and $c i s-\left[\mathrm{Ru}\left(\eta^{1}-\mathrm{OAc}\right)_{2}(\mathrm{D} i \mathrm{PPF})(\mathrm{ampy})\right](\mathbf{5 b})$ in $\mathrm{CD}_{3} \mathrm{OD}$

Pag. S25

Figure S22. ${ }^{1} \mathrm{H}$ NMR spectrum of the mixture $4: 1$ of the cationic species $\left[\operatorname{Ru}\left(\eta^{2}-\right.\right.$ $\mathrm{OAc})(\mathrm{D} i \mathrm{PPF})(\mathrm{ampy})] \mathrm{OAc}(\mathbf{5 a})$ and $c i s-\left[\mathrm{Ru}\left(\eta^{1}-\mathrm{OAc}\right)_{2}(\mathrm{D} i \mathrm{PPF})(\mathrm{ampy})\right](\mathbf{5 b})$ in $\mathrm{CD}_{3} \mathrm{OD}$

Pag. S26

Figure S23. ${ }^{1} \mathrm{H}$ NMR spectrum of the mixture $4: 1$ of the cationic species $\left[\operatorname{Ru}\left(\eta^{2}-\right.\right.$ $\mathrm{OAc})(\mathrm{D} i \mathrm{PPF})(\mathrm{ampy})] \mathrm{OAc}(\mathbf{5 a})$ and $c i s-\left[\mathrm{Ru}\left(\eta^{1}-\mathrm{OAc}\right)_{2}(\mathrm{D} i \mathrm{PPF})(\mathrm{ampy})\right](\mathbf{5 b})$ after the addition of $\mathrm{NaOAc}$ (3.5 equiv) in $\mathrm{CD}_{3} \mathrm{OD}$ Pag. S27

Figure S24. Effect of the addition of NaOAc (3.5 equiv) to the mixture 4:1 of the cationic species $\left[\mathrm{Ru}\left(\eta^{2}-\mathrm{OAc}\right)(\mathrm{D} i \mathrm{PPF})(\mathrm{ampy})\right] \mathrm{OAc}(\mathbf{5 a})$ and $c i s-\left[\mathrm{Ru}\left(\eta^{1}-\mathrm{OAc}\right)_{2}(\mathrm{D} i \mathrm{PPF})(\mathrm{ampy})\right](\mathbf{5 b})$ in the methyl acetate region of the ${ }^{1} \mathrm{H} \mathrm{NMR}$ spectrum in $\mathrm{CD}_{3} \mathrm{OD}$ Pag. S28

Figure S25. ${ }^{31} \mathrm{P}\left\{{ }^{1} \mathrm{H}\right\}$ NMR spectrum of trans-[Ru( $\left.\left.\eta^{1}-\mathrm{OAc}\right)_{2}(\mathrm{DCyPF})(\operatorname{ampy})\right](6) \quad$ Pag. S29 Figure S26. ${ }^{1} \mathrm{H}$ NMR spectrum of trans-[Ru( $\left.\left.\eta^{1}-\mathrm{OAc}\right)_{2}(\mathrm{DCyPF})(\mathrm{ampy})\right](\mathbf{6}) \quad$ Pag. S30 Figure S27. ${ }^{13} \mathrm{C}\left\{{ }^{1} \mathrm{H}\right\}$ PENDANT NMR spectrum of trans $-\left[\mathrm{Ru}\left(\eta^{1}-\mathrm{OAc}\right)_{2}(\mathrm{DCyPF})(\operatorname{ampy})\right](\mathbf{6})$

Pag. S31

Figure S28. ${ }^{31} \mathrm{P}\left\{{ }^{1} \mathrm{H}\right\}$ NMR spectrum of the mixture of trans-[Ru( $\left.\left.\eta^{1}-\mathrm{OAc}\right)_{2}(\mathrm{DCyPF})(\operatorname{ampy})\right](6)$ and the cationic species $\left[\mathrm{Ru}\left(\eta^{2}-\mathrm{OAc}\right)(\mathrm{DCyPF})(\operatorname{ampy})\right] \mathrm{OAc}(7)$ (in a 3:2 ratio) in $\mathrm{CDCl}_{3} \quad$ Pag. S32 Figure S29. ${ }^{31} \mathrm{P}\left\{{ }^{1} \mathrm{H}\right\} \mathrm{NMR}$ spectrum of the mixture of trans-[Ru( $\left.\left(\eta^{1}-\mathrm{OAc}\right)_{2}(\mathrm{DCyPF})(\operatorname{ampy})\right](6)$ and the cationic species $\left[\mathrm{Ru}\left(\eta^{2}-\mathrm{OAc}\right)(\mathrm{DCyPF})(\operatorname{ampy})\right] \mathrm{OAc}(7)$ (in a 3:2 ratio) in toluene- $d^{8} \mathrm{Pag}$. S33 Figure S30. ${ }^{1} \mathrm{H}$ NMR spectrum of the mixture of trans- $\left[\mathrm{Ru}\left(\eta^{1}-\mathrm{OAc}\right)_{2}(\mathrm{DCyPF})(\operatorname{ampy})\right](6)$ and the cationic species $\left[\mathrm{Ru}\left(\eta^{2}-\mathrm{OAc}\right)(\mathrm{DCyPF})(\mathrm{ampy})\right] \mathrm{OAc}(7)$ (in a 3:2 ratio) in toluene- $d^{8} \quad$ Pag. S34 Figure S31. ${ }^{31} \mathrm{P}\left\{{ }^{1} \mathrm{H}\right\}$ NMR spectrum of $\left[\mathrm{Ru}\left(\eta^{1}-\mathrm{OAc}\right)\left(\eta^{2}-\mathrm{OAc}\right)(\mathrm{DCyPP})(\mathrm{CO})\right](\mathbf{8}) \quad$ Pag. S35 Figure S32. ${ }^{1} \mathrm{H}$ NMR spectrum of $\left[\mathrm{Ru}\left(\eta^{1}-\mathrm{OAc}\right)\left(\eta^{2}-\mathrm{OAc}\right)(\mathrm{DCyPP})(\mathrm{CO})\right](\mathbf{8}) \quad$ Pag. S36 Figure S33. ${ }^{13} \mathrm{C}\left\{{ }^{1} \mathrm{H}\right\}$ DEPTQ NMR spectrum of $\left[\mathrm{Ru}\left(\eta^{1}-\mathrm{OAc}\right)\left(\eta^{2}-\mathrm{OAc}\right)(\mathrm{DCyPP})(\mathrm{CO})\right](\mathbf{8})$ 
Figure S34. ${ }^{31} \mathrm{P}\left\{{ }^{1} \mathrm{H}\right\}$ NMR spectrum of $\left[\mathrm{Ru}\left(\eta^{1}-\mathrm{OAc}\right)\left(\eta^{2}-\mathrm{OAc}\right)\left(\mathrm{Josiphos}^{\mathrm{cy}}\right)(\mathrm{CO})\right](9) \quad$ Pag. S38

Figure S35. ${ }^{1} \mathrm{H}$ NMR spectrum of $\left[\mathrm{Ru}\left(\eta^{1}-\mathrm{OAc}\right)\left(\eta^{2}-\mathrm{OAc}\right)\left(\mathrm{Josiphos}^{\mathrm{cy}}\right)(\mathrm{CO})\right](9) \quad$ Pag. S39

Figure S36. ${ }^{13} \mathrm{C}\left\{{ }^{1} \mathrm{H}\right\}$ DEPTQ NMR spectrum of $\left[\mathrm{Ru}\left(\eta^{1}-\mathrm{OAc}\right)\left(\eta^{2}-\mathrm{OAc}\right)\left(\mathrm{Josiphos}^{\mathrm{cy}}\right)(\mathrm{CO})\right](9)$

Pag. S40

Figure S37. ${ }^{31} \mathrm{P}\left\{{ }^{1} \mathrm{H}\right\}$ NMR spectrum of $\left[\mathrm{Ru}\left(\eta^{2}-\mathrm{OAc}\right)(\mathrm{C} \equiv \mathrm{CPh})(\mathrm{D} i \mathrm{PPF})(\mathrm{CO})\right](\mathbf{1 1}) \quad$ Pag. S41

Figure S38. ${ }^{1} \mathrm{H} N \mathrm{NMR}$ spectrum of $\left[\mathrm{Ru}\left(\eta^{2}-\mathrm{OAc}\right)(\mathrm{C} \equiv \mathrm{CPh})(\mathrm{D} i \mathrm{PPF})(\mathrm{CO})\right](\mathbf{1 1}) \quad$ Pag. S42

Figure S39. ${ }^{13} \mathrm{C}\left\{{ }^{1} \mathrm{H}\right\}$ PENDANT NMR spectrum of $\left[\mathrm{Ru}\left(\eta^{2}-\mathrm{OAc}\right)(\mathrm{C} \equiv \mathrm{CPh})(\mathrm{D} i \mathrm{PPF})(\mathrm{CO})\right](\mathbf{1 1})$

Pag. S43

Figure S40. ${ }^{31} \mathrm{P}\left\{{ }^{1} \mathrm{H}\right\}$ NMR spectrum of $\left[\mathrm{Ru}\left(\eta^{1}-\mathrm{OCOCF}_{3}\right)\left(\eta^{2}-\mathrm{OCOCF}_{3}\right)(\mathrm{D} i \mathrm{PPF})(\mathrm{CO})\right](\mathbf{1 2})$

Pag. S44

Figure S41. ${ }^{19} \mathrm{~F}\left\{{ }^{1} \mathrm{H}\right\}$ NMR spectrum of $\left[\mathrm{Ru}\left(\eta^{1}-\mathrm{OCOCF}_{3}\right)\left(\eta^{2}-\mathrm{OCOCF}_{3}\right)(\mathrm{D} i \mathrm{PPF})(\mathrm{CO})\right](\mathbf{1 2})$

Pag. S45

Figure S42. ${ }^{1} \mathrm{H}$ NMR spectrum of $\left[\mathrm{Ru}\left(\eta^{1}-\mathrm{OCOCF}_{3}\right)\left(\eta^{2}-\mathrm{OCOCF}_{3}\right)(\mathrm{D} i \mathrm{PPF})(\mathrm{CO})\right](\mathbf{1 2}) \quad$ Pag. S46

Figure S43. ${ }^{13} \mathrm{C}\left\{{ }^{1} \mathrm{H}\right\}$ DEPTQ NMR spectrum of $\left[\mathrm{Ru}\left(\eta^{1}-\mathrm{OCOCF}_{3}\right)\left(\eta^{2}-\mathrm{OCOCF}_{3}\right)(\mathrm{DiPPF})(\mathrm{CO})\right](\mathbf{1 2})$

Pag. S47

Figure S44. Evidences of the formation of mixed species $\left[\mathrm{Ru}(\mathrm{OAc})\left(\mathrm{OCOCF}_{3}\right)(\mathrm{CO})(\mathrm{DiPPF})\right]$ by reaction of complex $\mathbf{1 0}$ with TFA (1 equiv) in the ${ }^{31} \mathrm{P}\left\{{ }^{1} \mathrm{H}\right\}$ NMR spectrum Pag. S48

Figure S45. Evidences of the formation of mixed species $\left[\mathrm{Ru}(\mathrm{OAc})\left(\mathrm{OCOCF}_{3}\right)(\mathrm{CO})(\mathrm{D} i \mathrm{PPF})\right]$ by reaction of complex $\mathbf{1 0}$ with TFA (1 equiv) in the ${ }^{1} \mathrm{H}$ NMR spectrum Pag. S49

Figure S46. ${ }^{31} \mathrm{P}\left\{{ }^{1} \mathrm{H}\right\}$ NMR spectrum of trans, cis $-\left[\mathrm{Ru}\left(\eta^{1}-\mathrm{OAc}\right)_{2}(\mathrm{D} i \mathrm{PPF})(\mathrm{CO})_{2}\right](\mathbf{1 3}) \quad$ Pag. S50

Figure S47. ${ }^{1} \mathrm{H}$ NMR spectrum of trans, cis- $\left[\mathrm{Ru}\left(\eta^{1}-\mathrm{OAc}\right)_{2}(\mathrm{D} i \mathrm{PPF})(\mathrm{CO})_{2}\right](\mathbf{1 3}) \quad$ Pag. S51

Figure S48. ${ }^{13} \mathrm{C}\left\{{ }^{1} \mathrm{H}\right\}$ PENDANT NMR spectrum of trans, $c i s-\left[\mathrm{Ru}\left(\eta^{1}-\mathrm{OAc}\right)_{2}(\mathrm{D} i \mathrm{PPF})(\mathrm{CO})_{2}\right](\mathbf{1 3})$

Figure S49. ${ }^{31} \mathrm{P}\left\{{ }^{1} \mathrm{H}\right\}$ NMR spectrum of $c i s, c i s-\left[\mathrm{Ru}\left(\eta^{1}-\mathrm{OAc}\right)_{2}(\mathrm{D} i \mathrm{PPF})(\mathrm{CO})_{2}\right](\mathbf{1 4}) \quad$ Pag. S53 
Figure S50. ${ }^{1} \mathrm{H}$ NMR spectrum of $c i s, c i s-\left[\mathrm{Ru}\left(\eta^{1}-\mathrm{OAc}\right)_{2}(\mathrm{D} i \mathrm{PPF})(\mathrm{CO})_{2}\right](\mathbf{1 4})$

Pag. S54

Figure S51. ${ }^{13} \mathrm{C}\left\{{ }^{1} \mathrm{H}\right\}$ PENDANT NMR spectrum of $c i s, c i s-\left[\mathrm{Ru}\left(\eta^{1}-\mathrm{OAc}\right)_{2}(\mathrm{DiPPF})(\mathrm{CO})_{2}\right](\mathbf{1 4})$

Pag. S55

Figure S52. Representative ${ }^{1} \mathrm{H}$ NMR spectrum of 1,4-diphenyl-1-buten-3-yne obtained from Headto-Head dimerization of phenylacetylene promoted by complex $\mathbf{1 0}$

Pag. S56

Figure S53. Representative ${ }^{13} \mathrm{C}\left\{{ }^{1} \mathrm{H}\right\}$ DEPTQ spectrum of 1,4-diphenyl-1-buten-3-yne obtained from Head-to-Head dimerization of phenylacetylene promoted by complex $\mathbf{1 0}$

Pag. S57

Figure S54. Representative ${ }^{1} \mathrm{H}$ NMR spectrum of 1,6-diphenyl-2-hexen-4-yne obtained from Headto-Head dimerization of 3-Phenyl-1-Propyne promoted by complex 10

Pag. S58

Figure S55. Representative ${ }^{13} \mathrm{C}\left\{{ }^{1} \mathrm{H}\right\}$ PENDANT NMR spectrum of 1,6-diphenyl-2-hexen-4-yne obtained from Head-to-Head dimerization of 3-Phenyl-1-Propyne promoted by complex 10

Pag. S59

Figure S56. ${ }^{31} \mathrm{P}\left\{{ }^{1} \mathrm{H}\right\}$ NMR spectrum of unpurified reaction mixture of dimerization of phenylacetylene after $40 \mathrm{~h}$ heating at reflux promoted by complex $\mathbf{1 0}$ at $\mathrm{S} / \mathrm{C} 30$ Pag. S60 Figure S57. Stacked ${ }^{1} \mathrm{H}$ NMR spectrum of unpurified reaction mixture of dimerization of phenylacetylene before the heating and after $40 \mathrm{~h}$ at reflux, promoted by complex $\mathbf{1 0}$ at $\mathrm{S} / \mathrm{C} 30$

Pag. S61

Single crystal X-ray structure determination of complex 11. General data. Pag. S62

Figure S58. Molecular structure of complex 11

Pag. S63

Single crystal X-ray structure determination of complex 11. Detailed crystallographic data.

Pag. S64

References

Pag. S66 


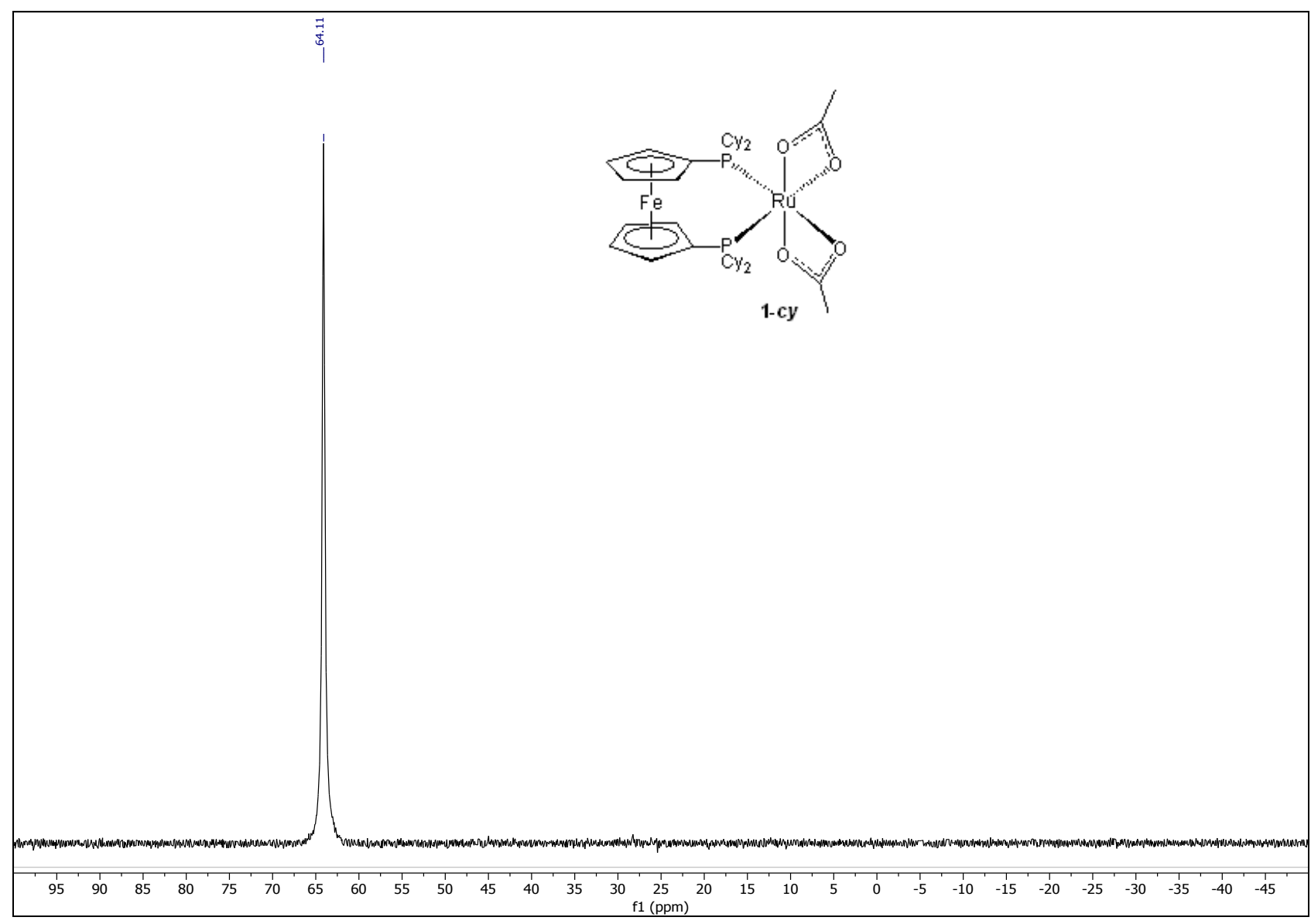

Figure S1. ${ }^{31} \mathrm{P}\left\{{ }^{1} \mathrm{H}\right\}$ NMR spectrum $(81.0 \mathrm{MHz})$ of $\left[\mathrm{Ru}\left(\eta^{2}-\mathrm{OAc}\right)_{2}(\mathrm{DCyPF})\right](\mathbf{1}-c y)$ in $\mathrm{CD}_{2} \mathrm{Cl}_{2}$ at 20 ${ }^{\circ} \mathrm{C}$. 


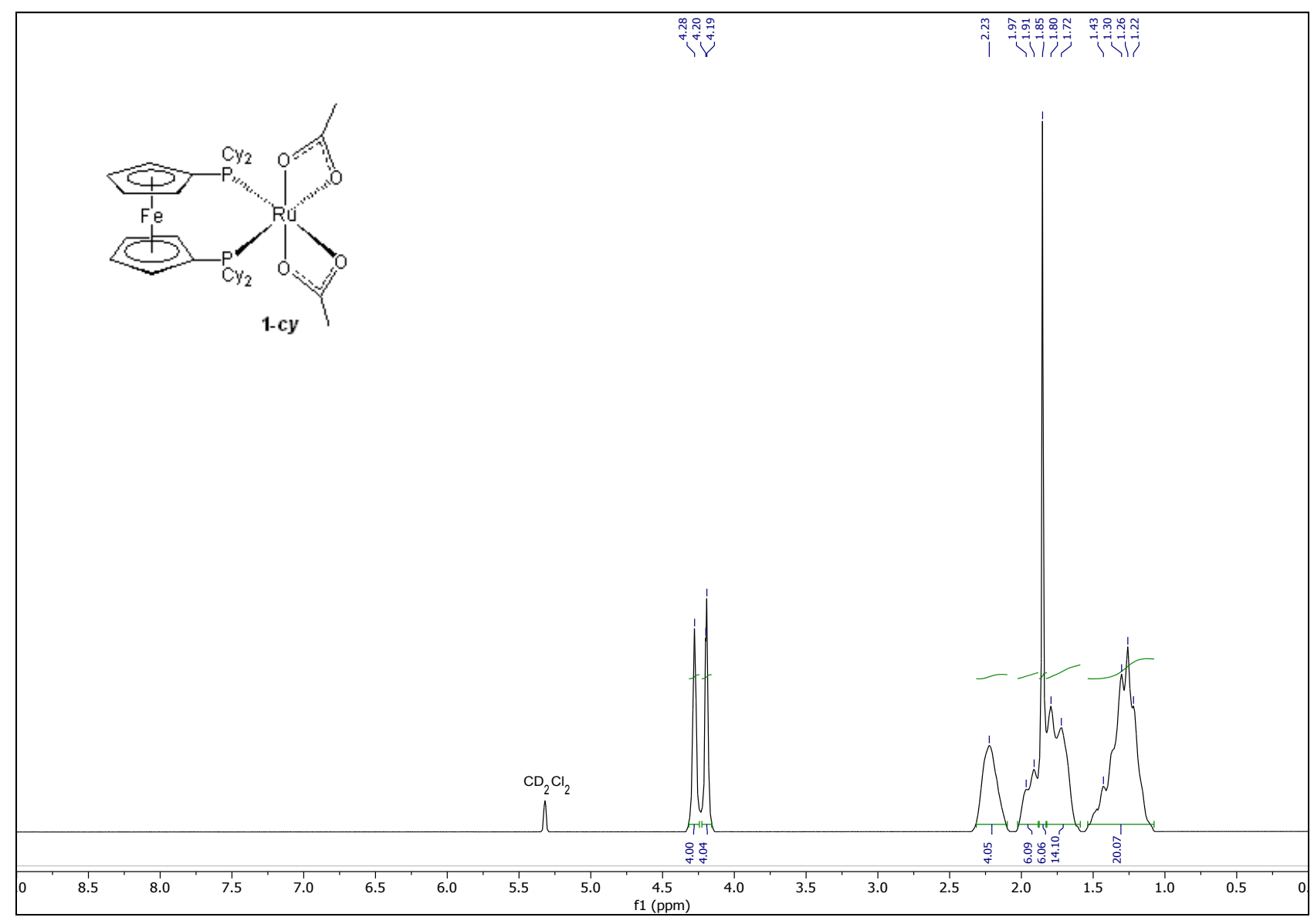

Figure S2. ${ }^{1} \mathrm{H}$ NMR spectrum $(200.1 \mathrm{MHz})$ of $\left[\mathrm{Ru}\left(\eta^{2}-\mathrm{OAc}\right)_{2}(\mathrm{DCyPF})\right](\mathbf{1}-\boldsymbol{c y})$ in $\mathrm{CD}_{2} \mathrm{Cl}_{2}$ at $20{ }^{\circ} \mathrm{C}$. 


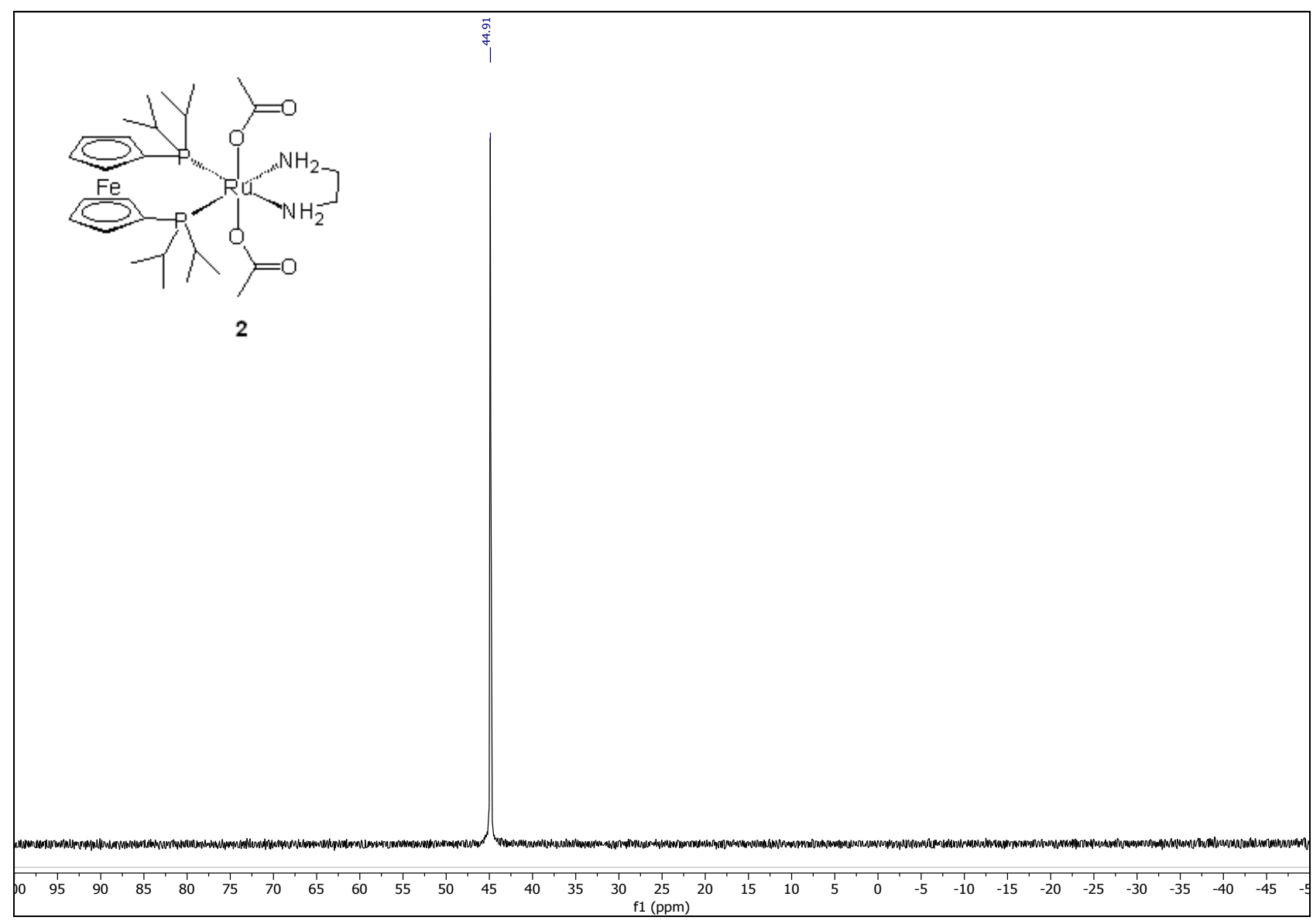

Figure S3. ${ }^{31} \mathrm{P}\left\{{ }^{1} \mathrm{H}\right\}$ NMR spectrum $(81.0 \mathrm{MHz})$ of trans-[Ru( $\left.\left.\eta^{1}-\mathrm{OAc}\right)_{2}(\mathrm{D} i \mathrm{PPF})(\mathrm{en})\right](2)$ in $\mathrm{CDCl}_{3}$ at $20^{\circ} \mathrm{C}$. 


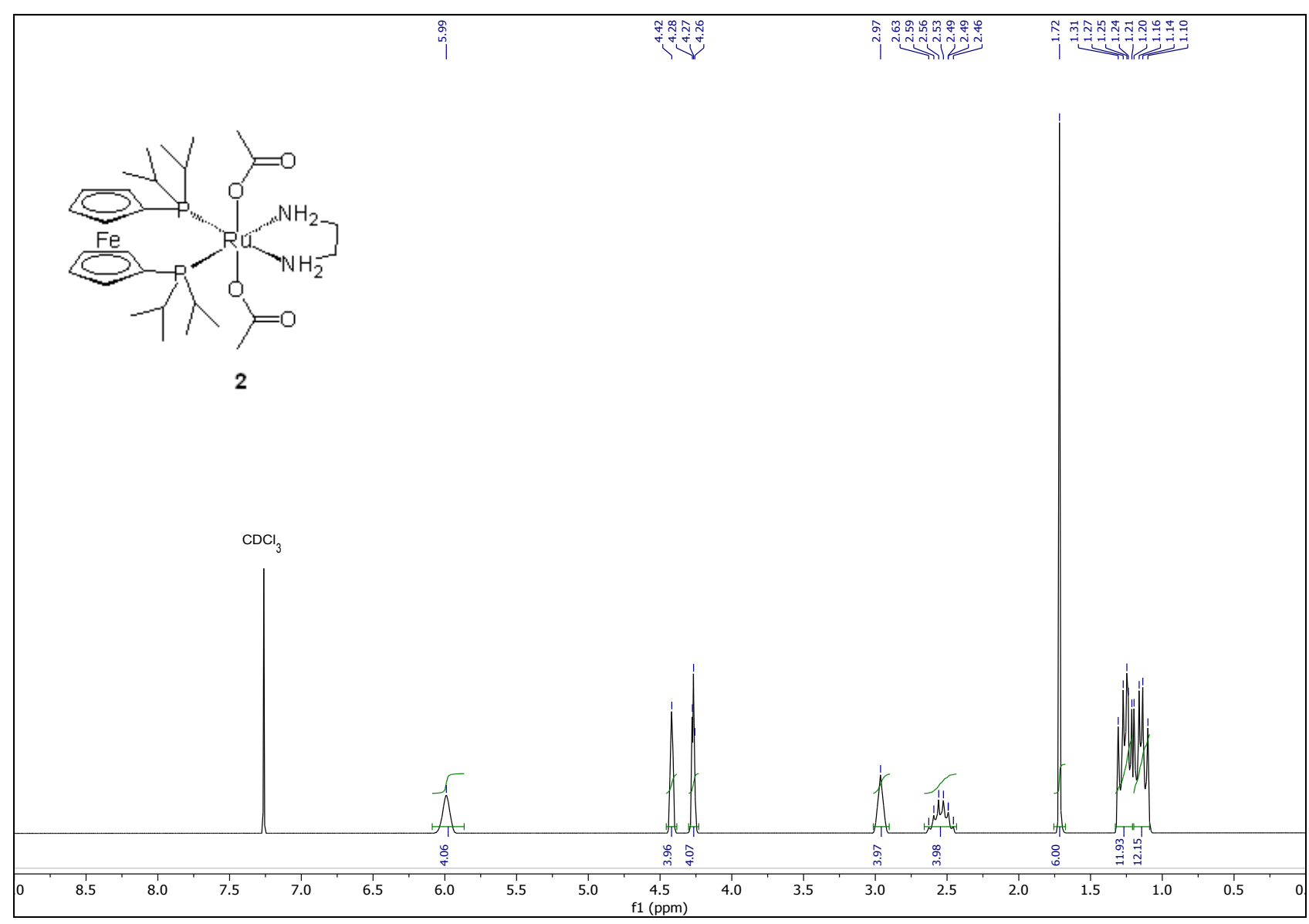

Figure S4. ${ }^{1} \mathrm{H}$ NMR spectrum $(200.1 \mathrm{MHz})$ of trans-[Ru( $\left.\left.\eta^{1}-\mathrm{OAc}\right)_{2}(\mathrm{D} i \mathrm{PPF})(\mathrm{en})\right](2)$ in $\mathrm{CDCl}_{3}$ at 20 ${ }^{\circ} \mathrm{C}$. 


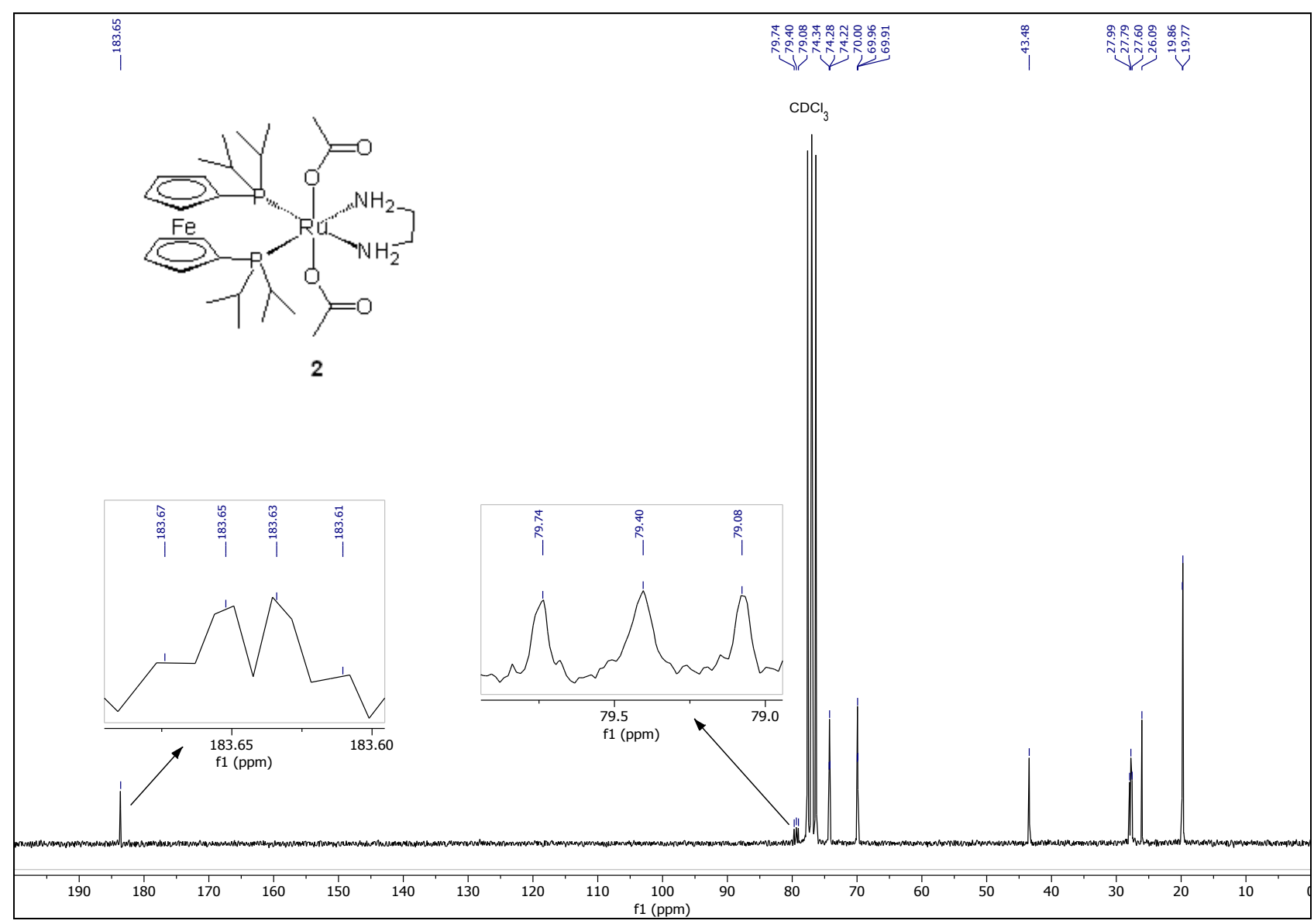

Figure S5. ${ }^{13} \mathrm{C}\left\{{ }^{1} \mathrm{H}\right\}$ NMR spectrum $(50.3 \mathrm{MHz})$ of trans-[Ru( $\left.\left.\eta^{1}-\mathrm{OAc}\right)_{2}(\mathrm{DiPPF})(\mathrm{en})\right](2)$ in $\mathrm{CDCl}_{3}$ at $20^{\circ} \mathrm{C}$. 


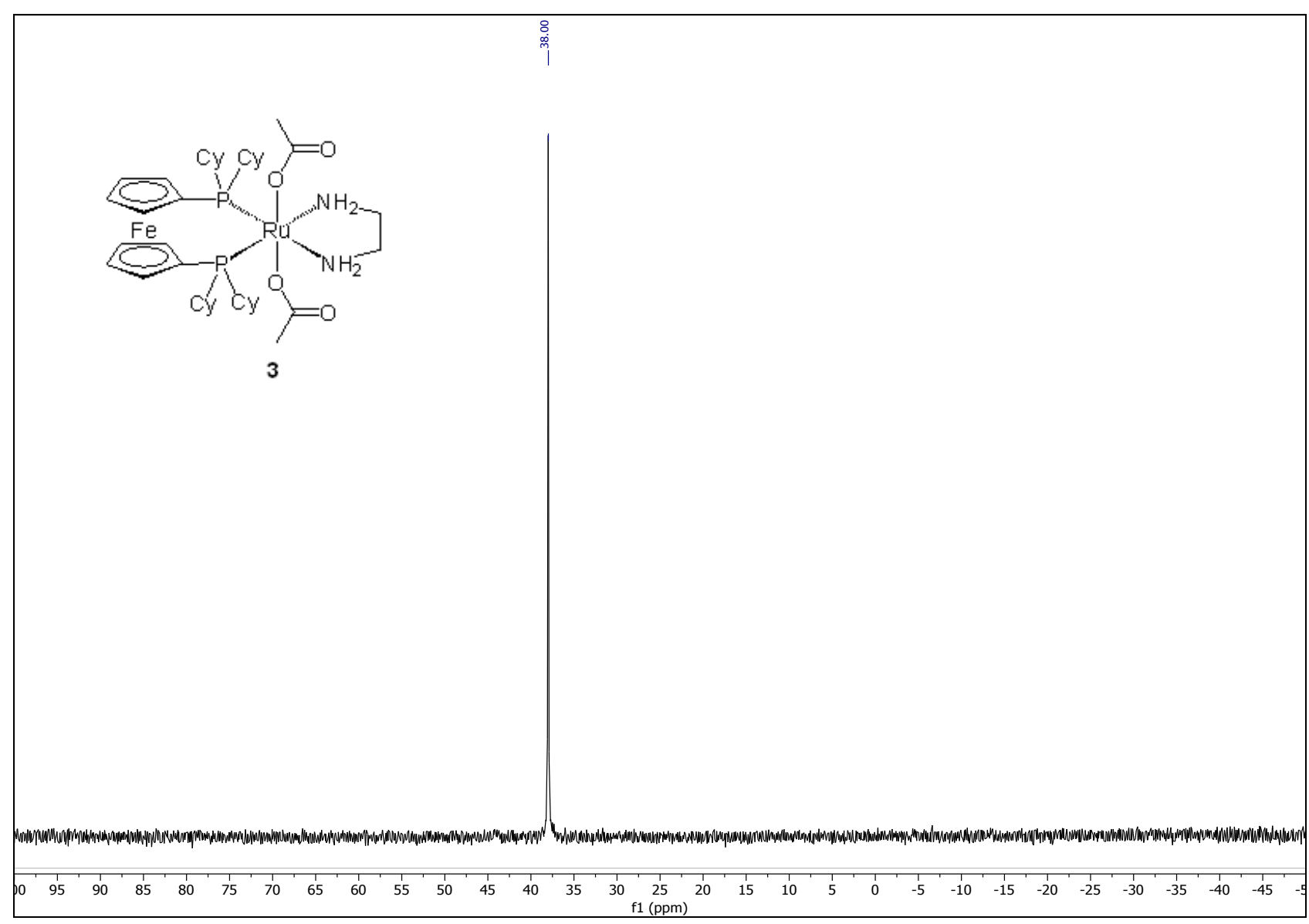

Figure S6. ${ }^{31} \mathrm{P}\left\{{ }^{1} \mathrm{H}\right\}$ NMR spectrum $(81.0 \mathrm{MHz})$ of trans-[Ru( $\left.\left.\eta^{1}-\mathrm{OAc}\right)_{2}(\mathrm{DCyPF})(\mathrm{en})\right](3)$ in $\mathrm{CD}_{2} \mathrm{Cl}_{2}$ at $20^{\circ} \mathrm{C}$. 


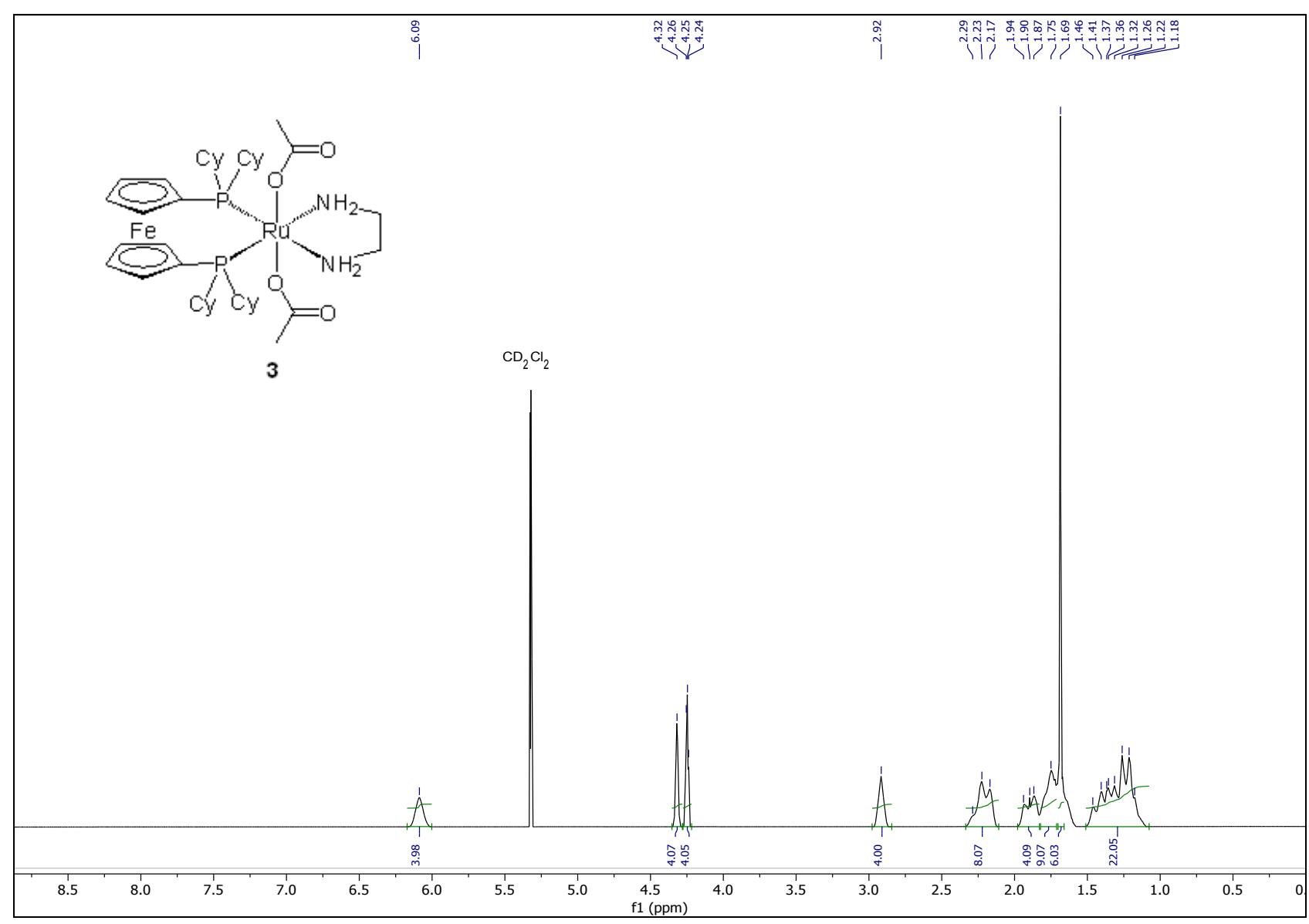

Figure S7. ${ }^{1} \mathrm{H}$ NMR spectrum $(200.1 \mathrm{MHz})$ of trans-[Ru( $\left.\left.\eta^{1}-\mathrm{OAc}\right)_{2}(\mathrm{DCyPF})(\mathrm{en})\right](3)$ in $\mathrm{CD}_{2} \mathrm{Cl}_{2}$ at 20 ${ }^{\circ} \mathrm{C}$. 


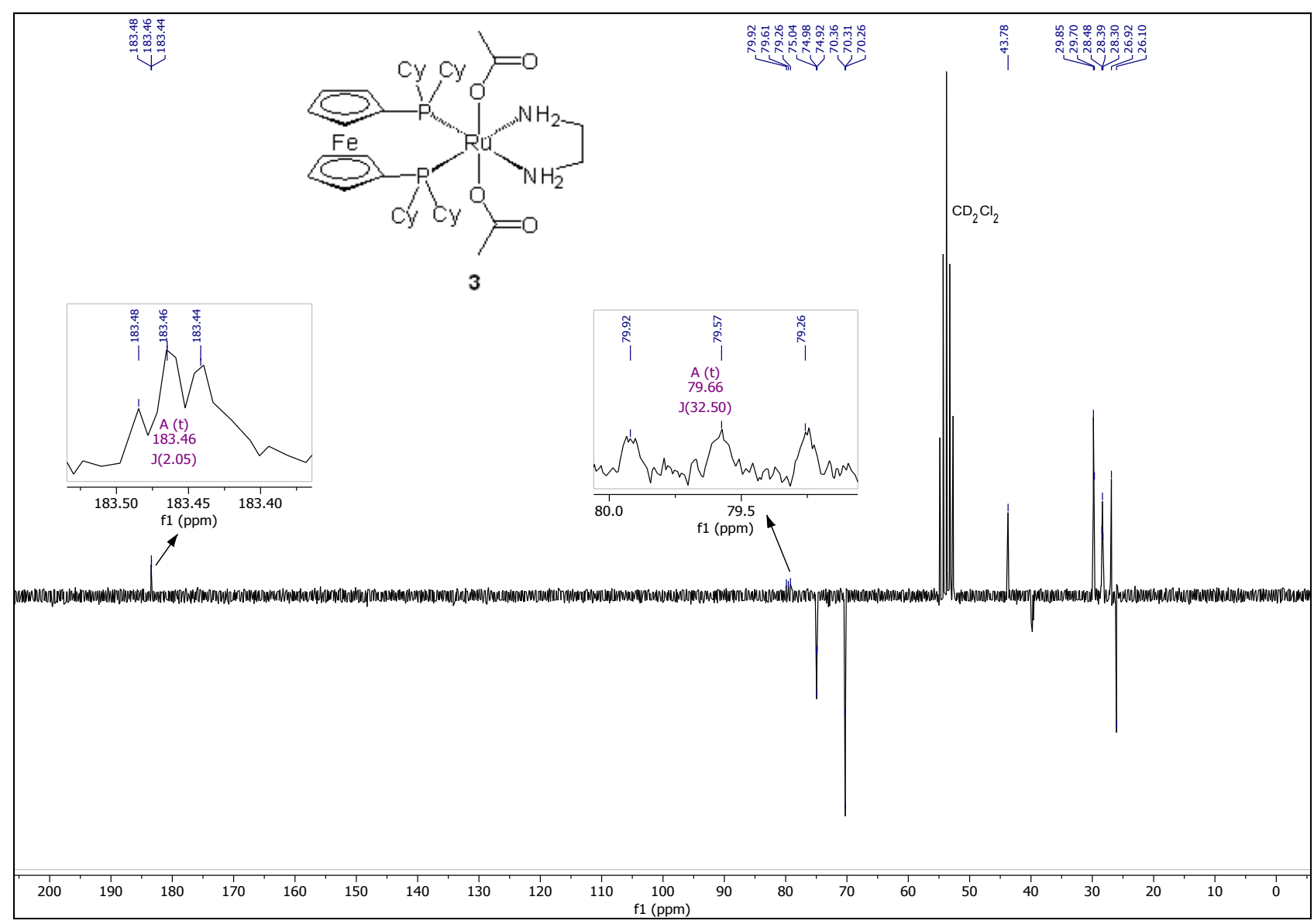

Figure S8. ${ }^{13} \mathrm{C}\left\{{ }^{1} \mathrm{H}\right\}$ PENDANT NMR spectrum $(50.3 \mathrm{MHz})$ of trans $-\left[\mathrm{Ru}\left(\eta^{1}-\mathrm{OAc}\right)_{2}(\mathrm{DCyPF})(\mathrm{en})\right](3)$ in $\mathrm{CD}_{2} \mathrm{Cl}_{2}$ at $20^{\circ} \mathrm{C}$. 


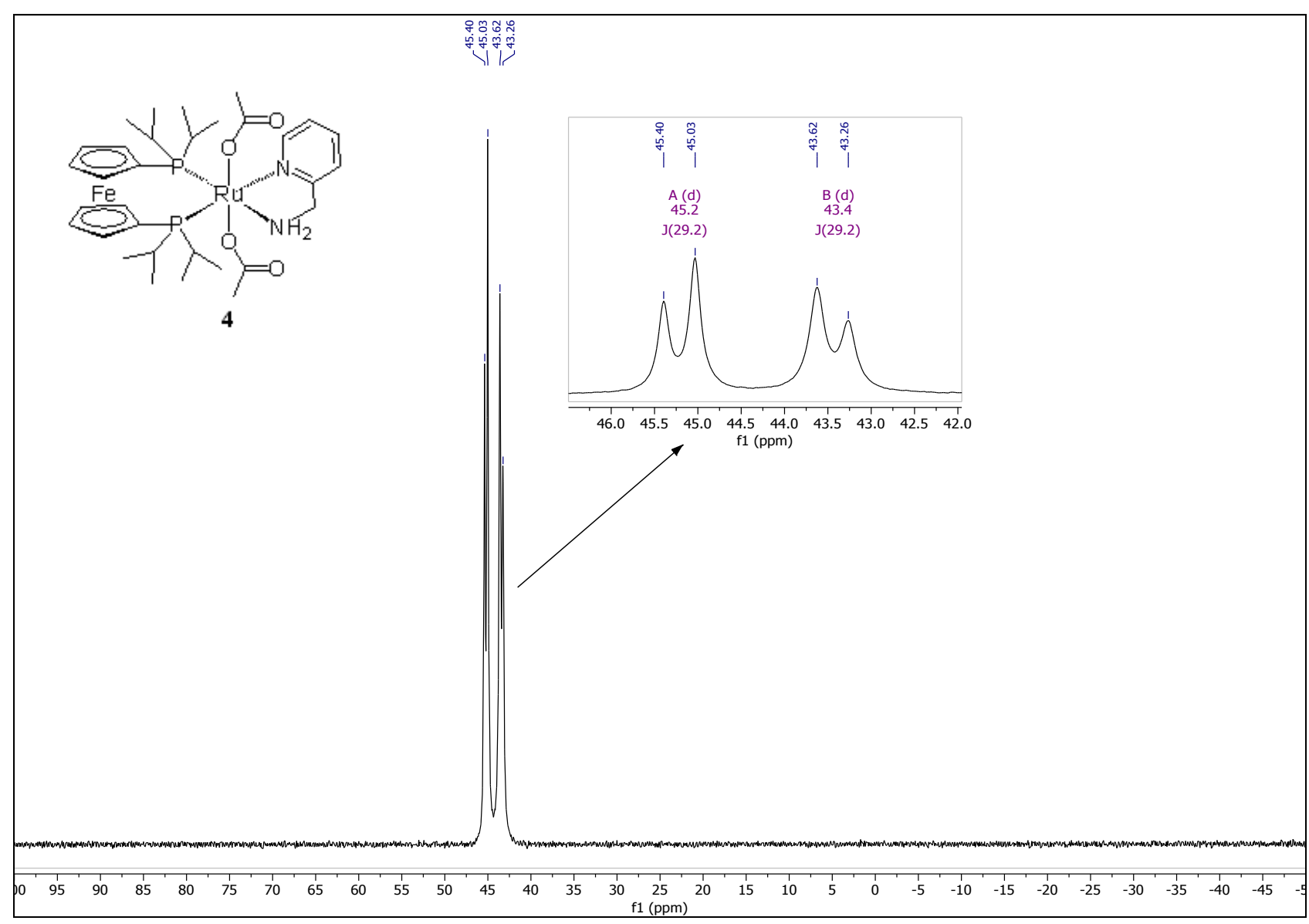

Figure S9. ${ }^{31} \mathrm{P}\left\{{ }^{1} \mathrm{H}\right\}$ NMR spectrum $(81.0 \mathrm{MHz})$ of trans-[Ru( $\left.\left.\eta^{1}-\mathrm{OAc}\right)_{2}(\mathrm{D} i \mathrm{PPF})(\operatorname{ampy})\right](4)$ in $\mathrm{CD}_{2} \mathrm{Cl}_{2}$ at $-60^{\circ} \mathrm{C}$. 


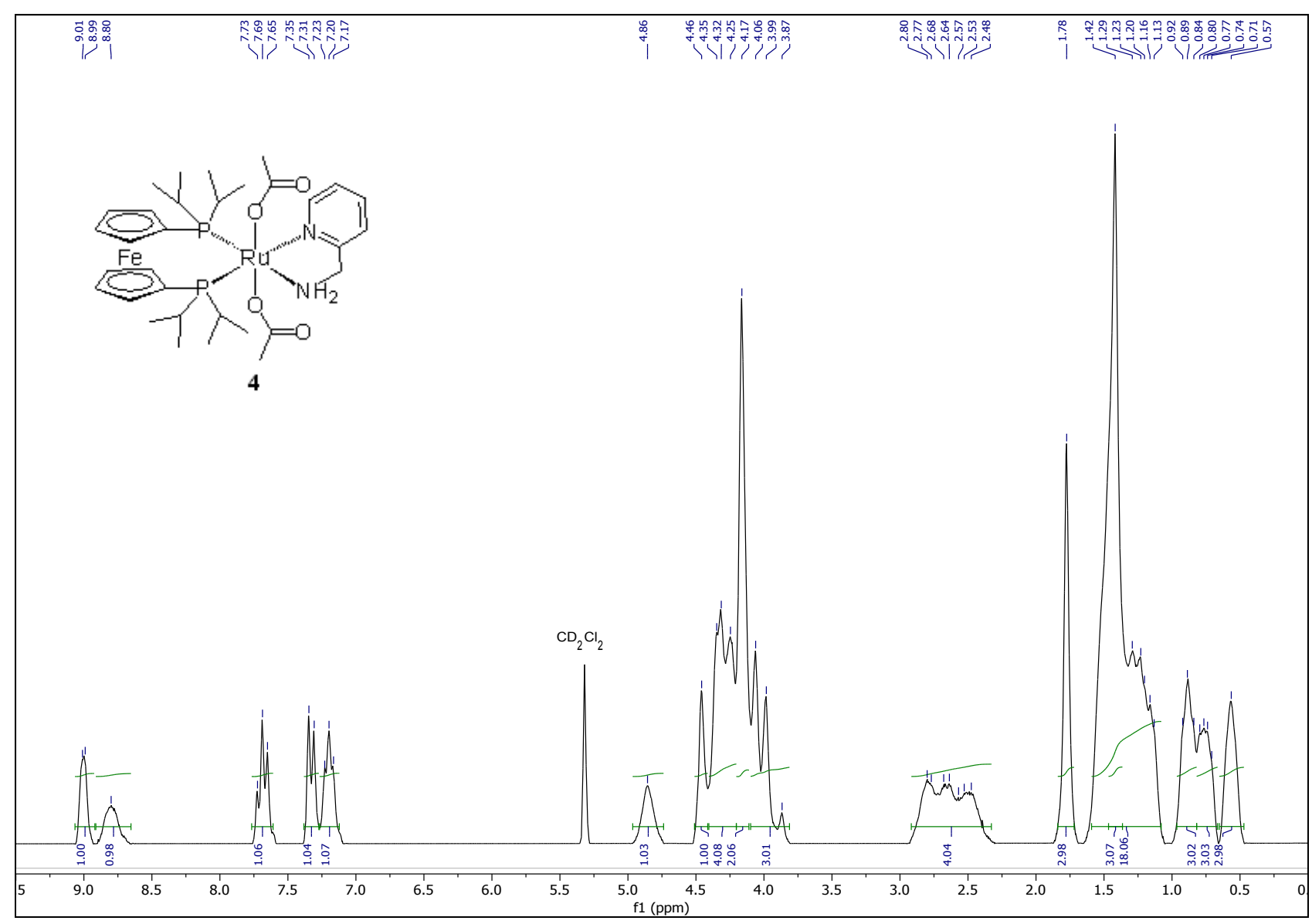

Figure S10. ${ }^{1} \mathrm{H}$ NMR spectrum $(200.1 \mathrm{MHz})$ of trans-[Ru( $\left.\left.\eta^{1}-\mathrm{OAc}\right)_{2}(\mathrm{DiPPF})(\operatorname{ampy})\right](4)$ in $\mathrm{CD}_{2} \mathrm{Cl}_{2}$ at $-60^{\circ} \mathrm{C}$. 


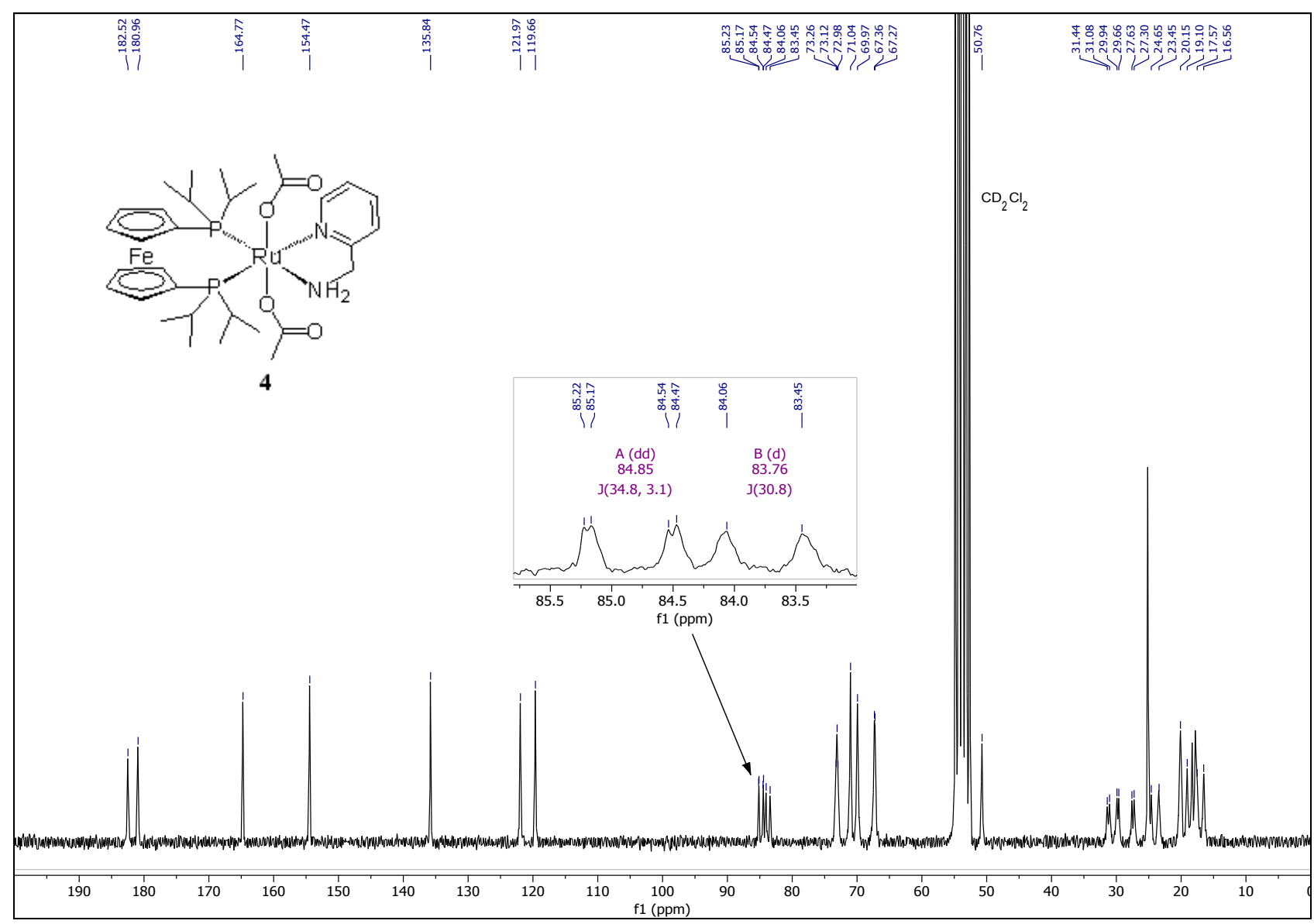

Figure S11. ${ }^{13} \mathrm{C}\left\{{ }^{1} \mathrm{H}\right\}$ NMR spectrum $(50.3 \mathrm{MHz})$ of trans-[Ru( $\left.\left.\eta^{1}-\mathrm{OAc}\right)_{2}(\mathrm{DiPPF})(\operatorname{ampy})\right](4)$ in $\mathrm{CD}_{2} \mathrm{Cl}_{2}$ at $-60{ }^{\circ} \mathrm{C}$. 


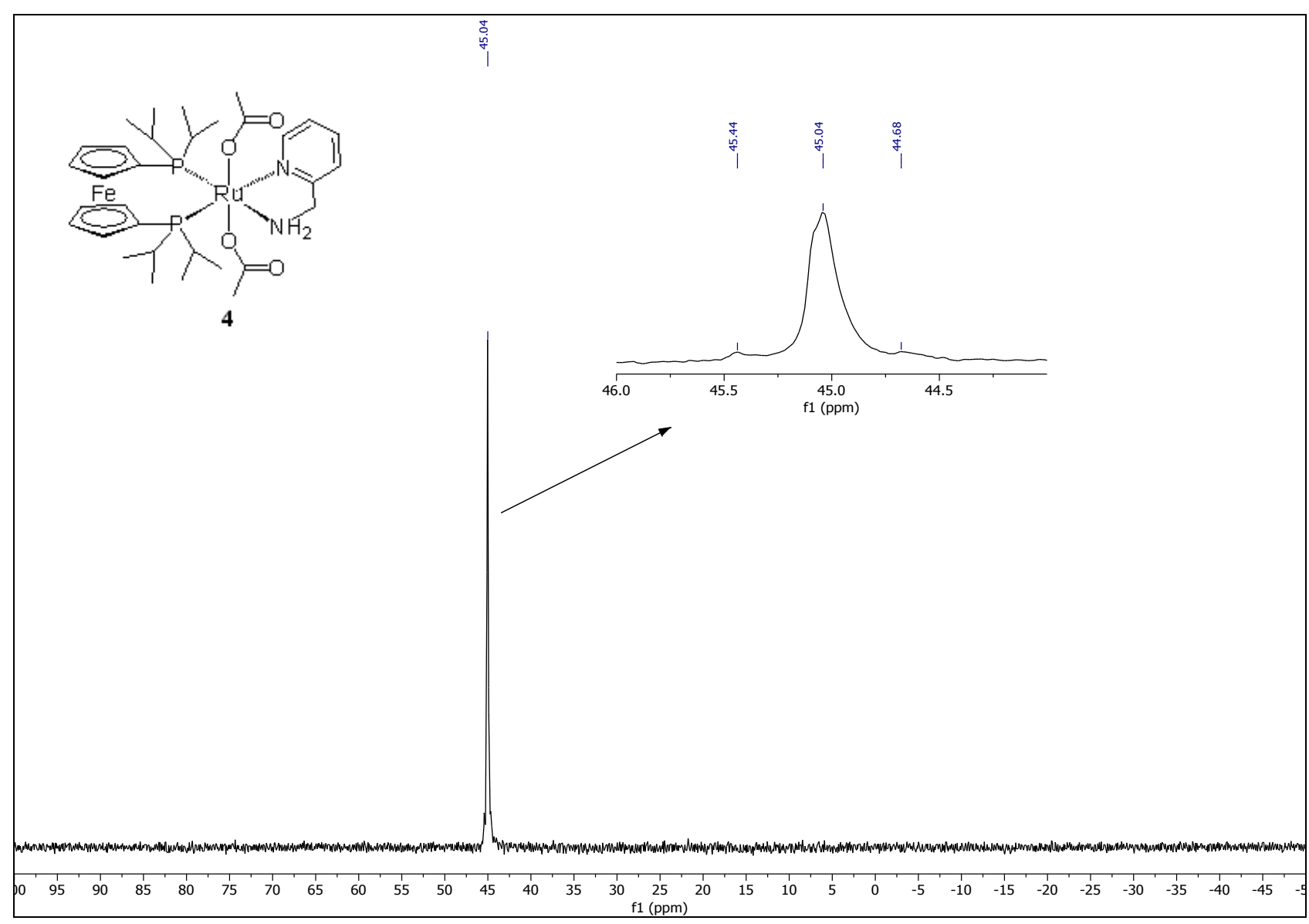

Figure S12. ${ }^{31} \mathrm{P}\left\{{ }^{1} \mathrm{H}\right\}$ NMR spectrum $(81.0 \mathrm{MHz})$ of trans $-\left[\mathrm{Ru}\left(\eta^{1}-\mathrm{OAc}\right)_{2}(\mathrm{D} i \mathrm{PPF})(\operatorname{ampy})\right]$ (4) in $\mathrm{CD}_{2} \mathrm{Cl}_{2}$ at $0{ }^{\circ} \mathrm{C}$. 


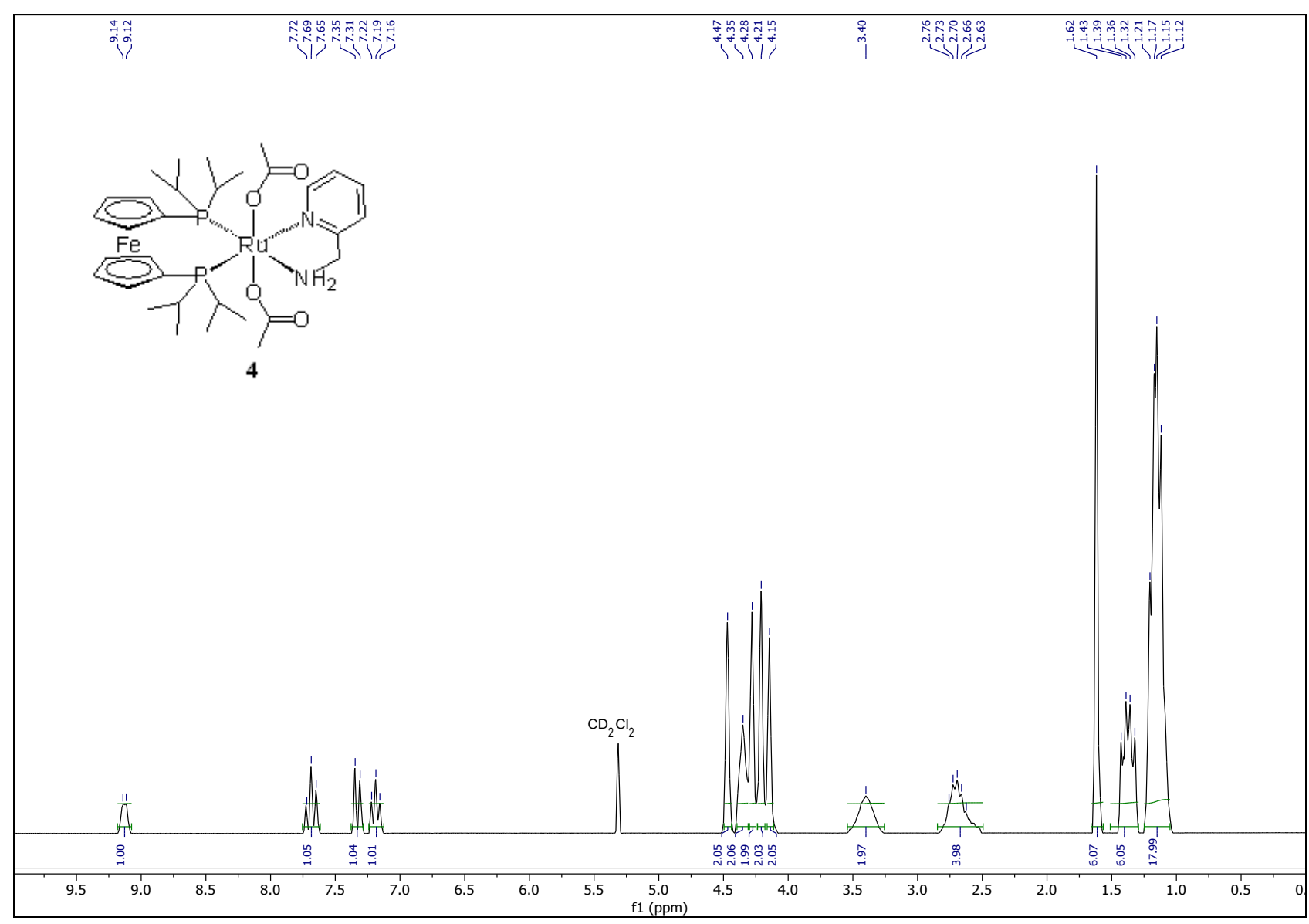

Figure S13. ${ }^{1} \mathrm{H}$ NMR spectrum $(200.1 \mathrm{MHz})$ of trans-[Ru( $\left.\left.\eta^{1}-\mathrm{OAc}\right)_{2}(\mathrm{DiPPF})(\operatorname{ampy})\right](4)$ in $\mathrm{CD}_{2} \mathrm{Cl}_{2}$ at $0{ }^{\circ} \mathrm{C}$. 


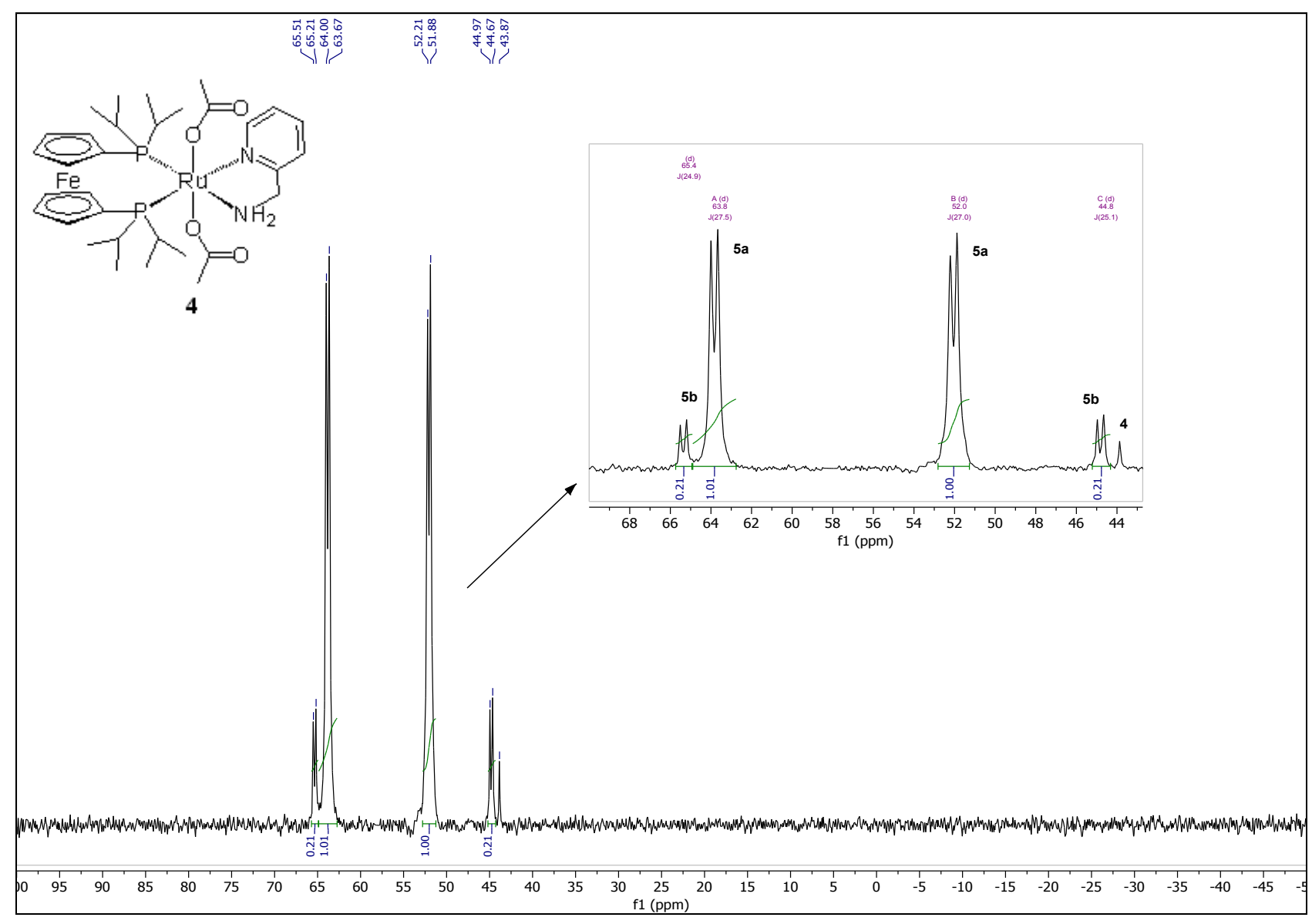

Figure S14. Effect of the heating on the solution of trans $-\left[\mathrm{Ru}\left(\eta^{1}-\mathrm{OAc}\right)_{2}(\mathrm{D} i \mathrm{PPF})(\mathrm{ampy})\right](4)$ in $\mathrm{CD}_{2} \mathrm{Cl}_{2}$ from $0^{\circ} \mathrm{C}$ to $\mathrm{RT}:{ }^{31} \mathrm{P}\left\{{ }^{1} \mathrm{H}\right\} \mathrm{NMR}$ spectrum $(81.0 \mathrm{MHz})$ after $24 \mathrm{~h}$. 


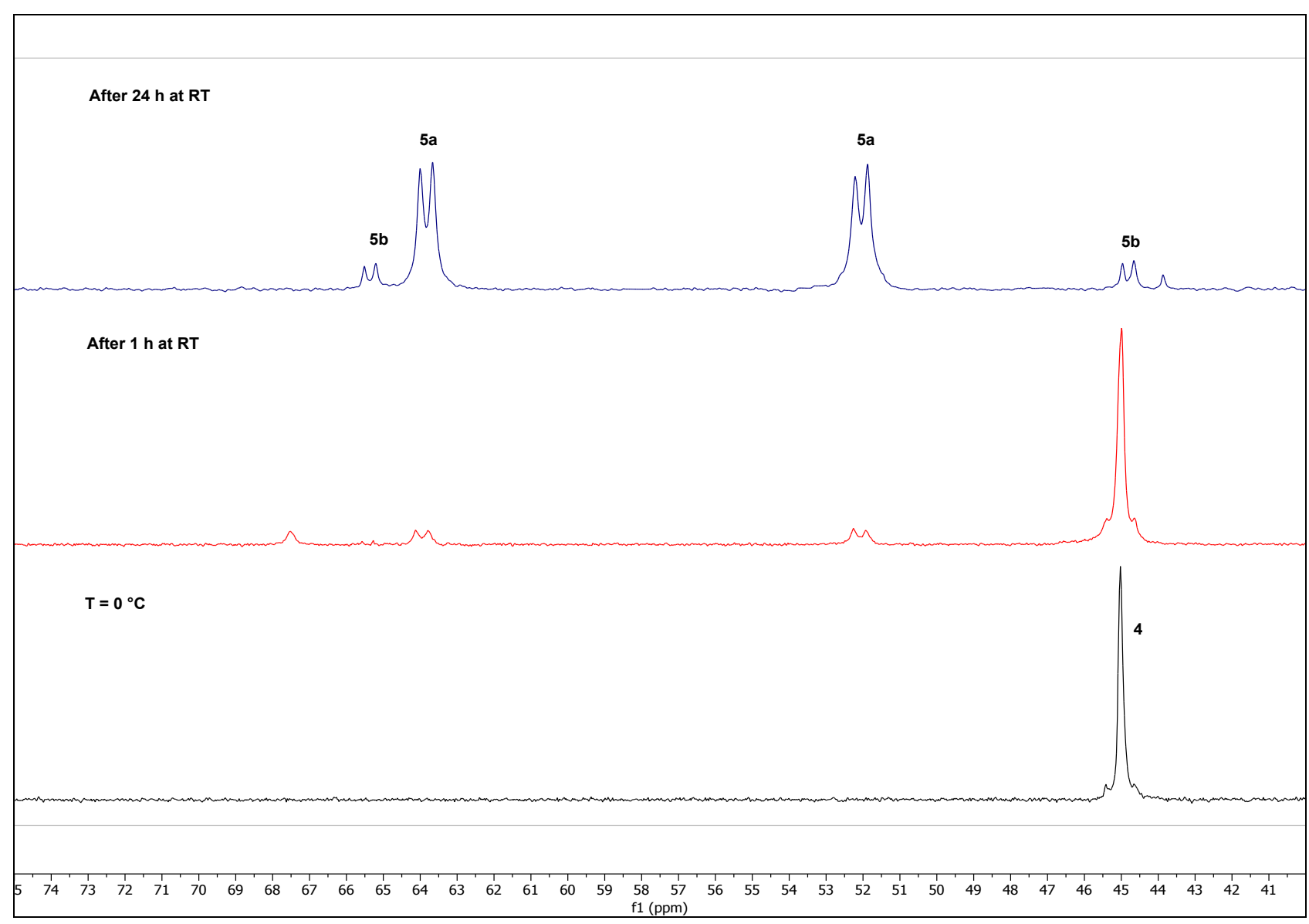

Figure S15. Stacked VT ${ }^{31} \mathrm{P}\left\{{ }^{1} \mathrm{H}\right\}$ NMR spectra $(81.0 \mathrm{MHz})$ from $0{ }^{\circ} \mathrm{C}$ to RT of the solution of trans$\left[\mathrm{Ru}\left(\eta^{1}-\mathrm{OAc}\right)_{2}(\mathrm{D} i \mathrm{PPF})(\right.$ ampy) $](4)$ in $\mathrm{CD}_{2} \mathrm{Cl}_{2}$. 


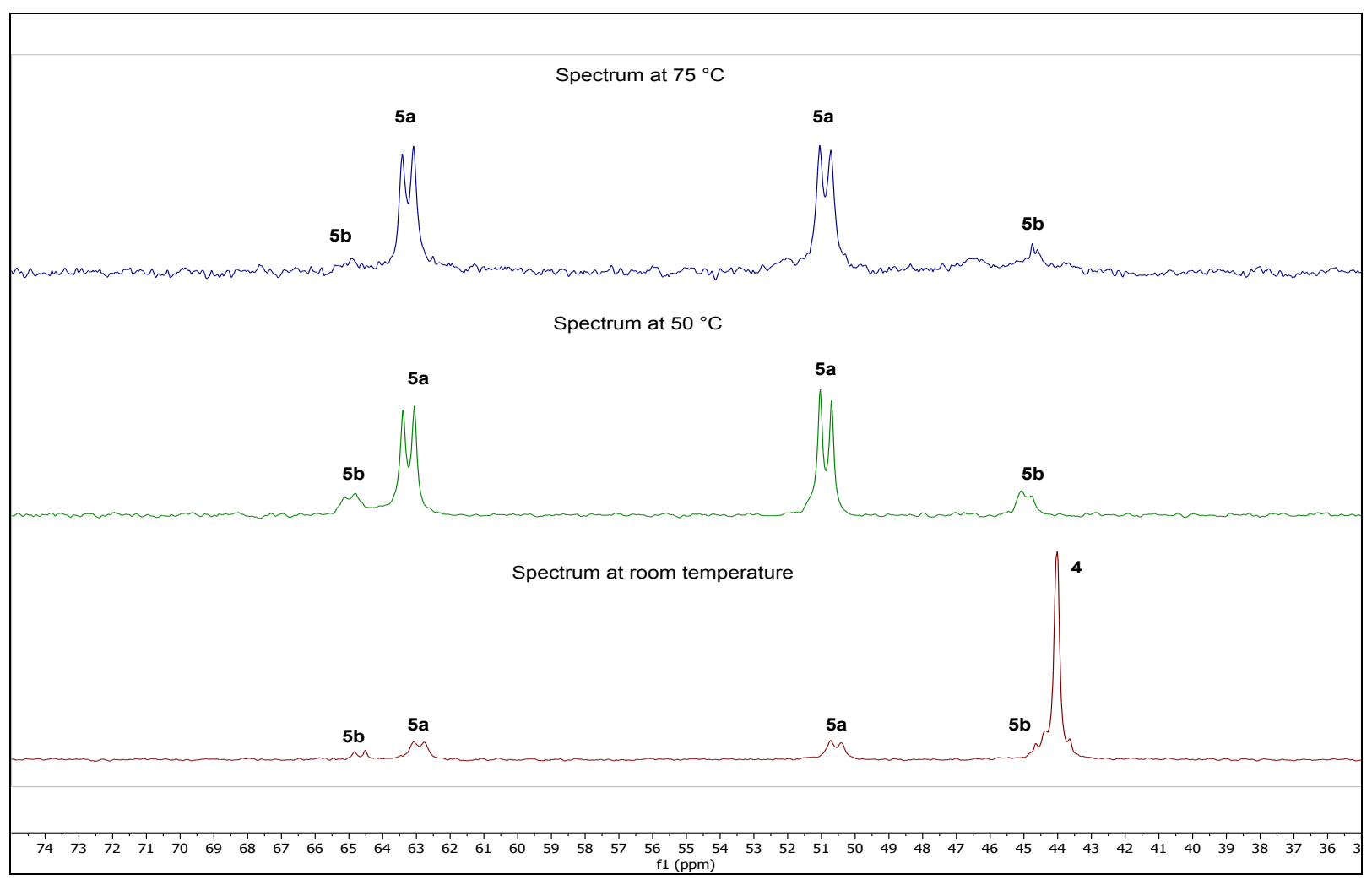

Figure S16. Stacked VT ${ }^{31} \mathrm{P}\left\{{ }^{1} \mathrm{H}\right\}$ NMR spectra $(81.0 \mathrm{MHz})$ from $\mathrm{RT}$ to $75{ }^{\circ} \mathrm{C}$ of the products of the reaction between $\left[\mathrm{Ru}\left(\eta^{1}-\mathrm{OAc}\right)\left(\eta^{2}-\mathrm{OAc}\right)(\mathrm{D} i \mathrm{PPF})\right](\mathbf{1}-\mathbf{i p})$ and ampy (3 equiv) in isopropanol- $d^{8}$.

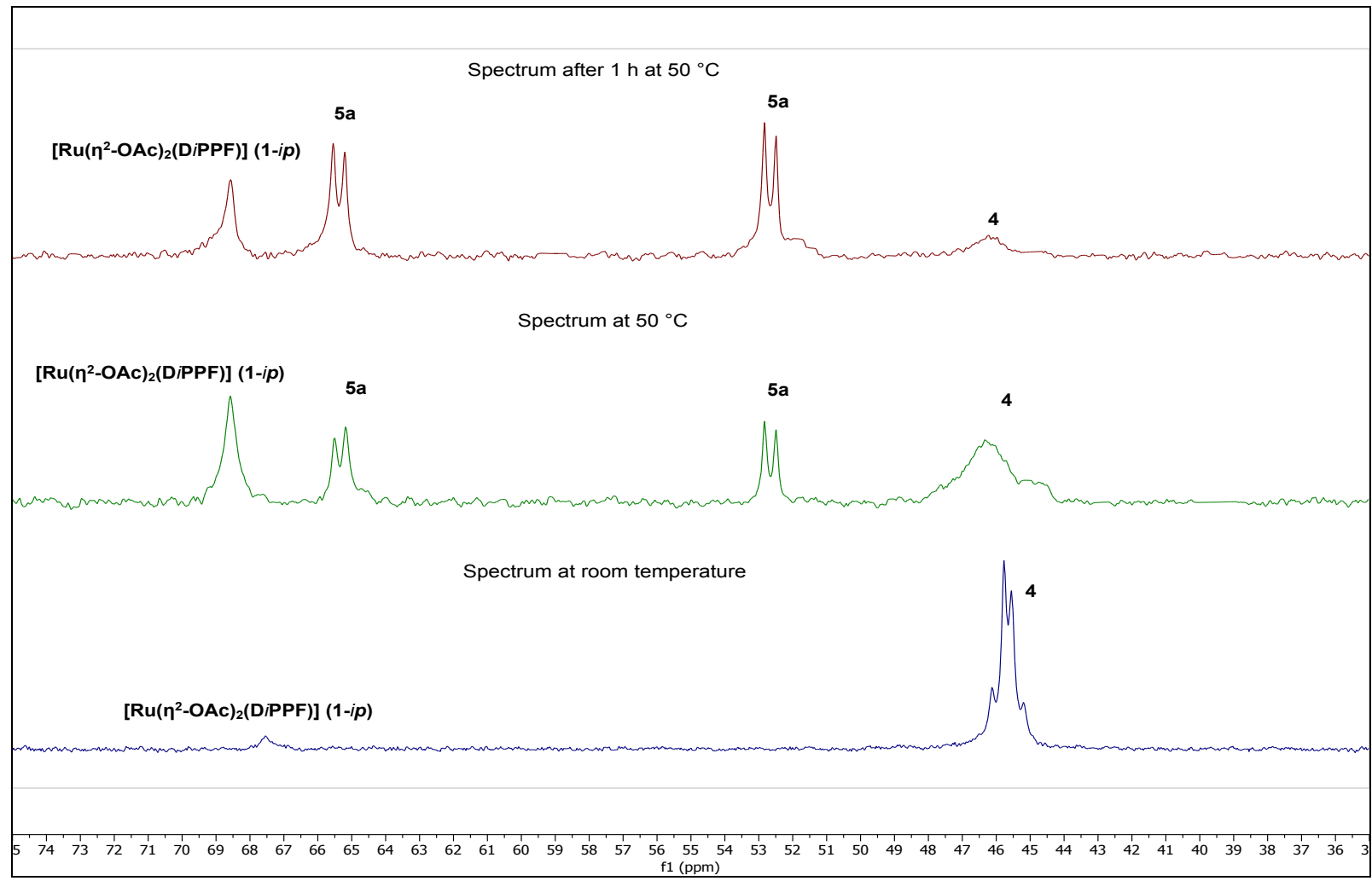

Figure S17. Stacked VT ${ }^{31} \mathrm{P}\left\{{ }^{1} \mathrm{H}\right\}$ NMR spectra $(81.0 \mathrm{MHz})$ from $\mathrm{RT}$ to $50{ }^{\circ} \mathrm{C}$ of the products of the reaction between $\left[\mathrm{Ru}\left(\eta^{1}-\mathrm{OAc}\right)\left(\eta^{2}-\mathrm{OAc}\right)(\mathrm{D} i \mathrm{PPF})\right](\mathbf{1}-\mathbf{i p})$ and ampy $\left(1.3\right.$ equiv) in toluene- $d^{8}$. 


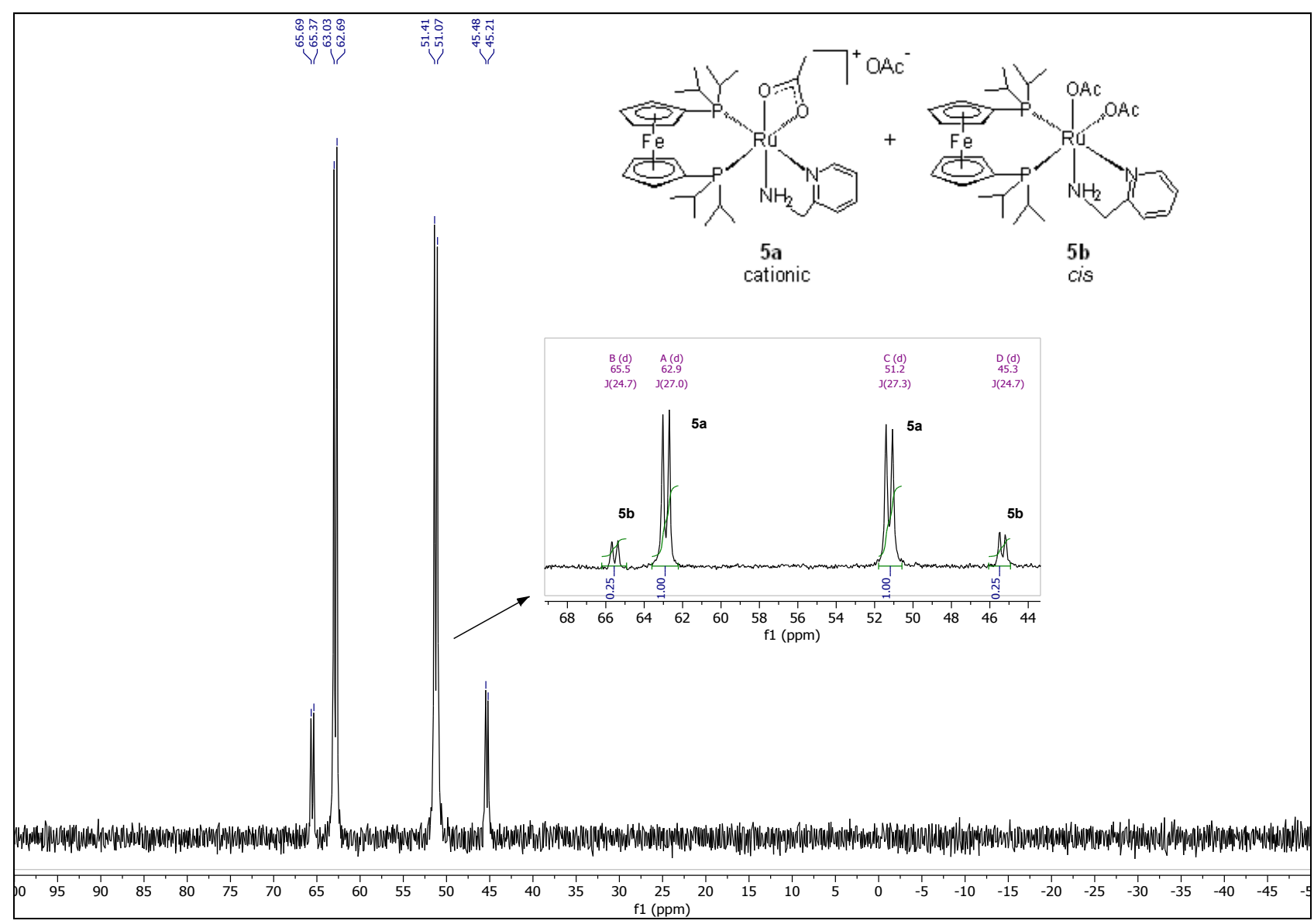

Figure S18. ${ }^{31} \mathrm{P}\left\{{ }^{1} \mathrm{H}\right\}$ NMR spectrum $(81.0 \mathrm{MHz})$ of the mixture $4: 1$ of the cationic species $\left[\mathrm{Ru}\left(\eta^{2}\right.\right.$ $\mathrm{OAc})(\mathrm{D} i \mathrm{PPF})(\mathrm{ampy})] \mathrm{OAc}(\mathbf{5 a})$ and $c i s-\left[\mathrm{Ru}\left(\eta^{1}-\mathrm{OAc}\right)_{2}(\mathrm{D} i \mathrm{PPF})(\mathrm{ampy})\right](\mathbf{5 b})$ in $\mathrm{CD}_{2} \mathrm{Cl}_{2} / \mathrm{CD}_{3} \mathrm{OD}$ at 20 ${ }^{\circ} \mathrm{C}$. 


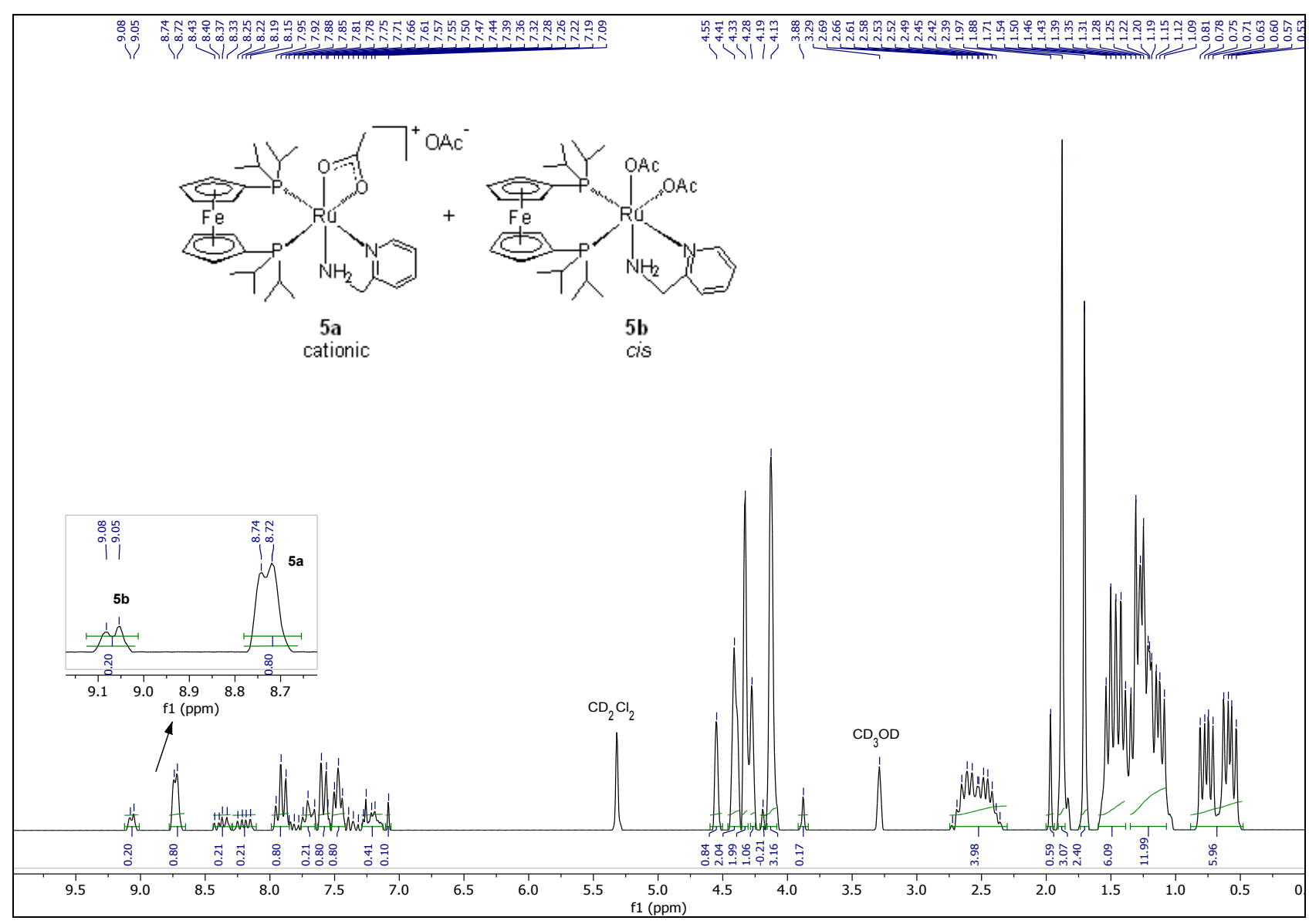

Figure S19. ${ }^{1} \mathrm{H}$ NMR spectrum $(200.1 \mathrm{MHz})$ of the mixture $4: 1$ of the cationic species $\left[\mathrm{Ru}\left(\eta^{2}\right.\right.$ $\mathrm{OAc})(\mathrm{D} i \mathrm{PPF})(\mathrm{ampy})] \mathrm{OAc}(\mathbf{5 a})$ and $c i s-\left[\mathrm{Ru}\left(\eta^{1}-\mathrm{OAc}\right)_{2}(\mathrm{D} i \mathrm{PPF})(\mathrm{ampy})\right](\mathbf{5 b})$ in $\mathrm{CD}_{2} \mathrm{Cl}_{2} / \mathrm{CD}_{3} \mathrm{OD}$ at 20 ${ }^{\circ} \mathrm{C}$. 


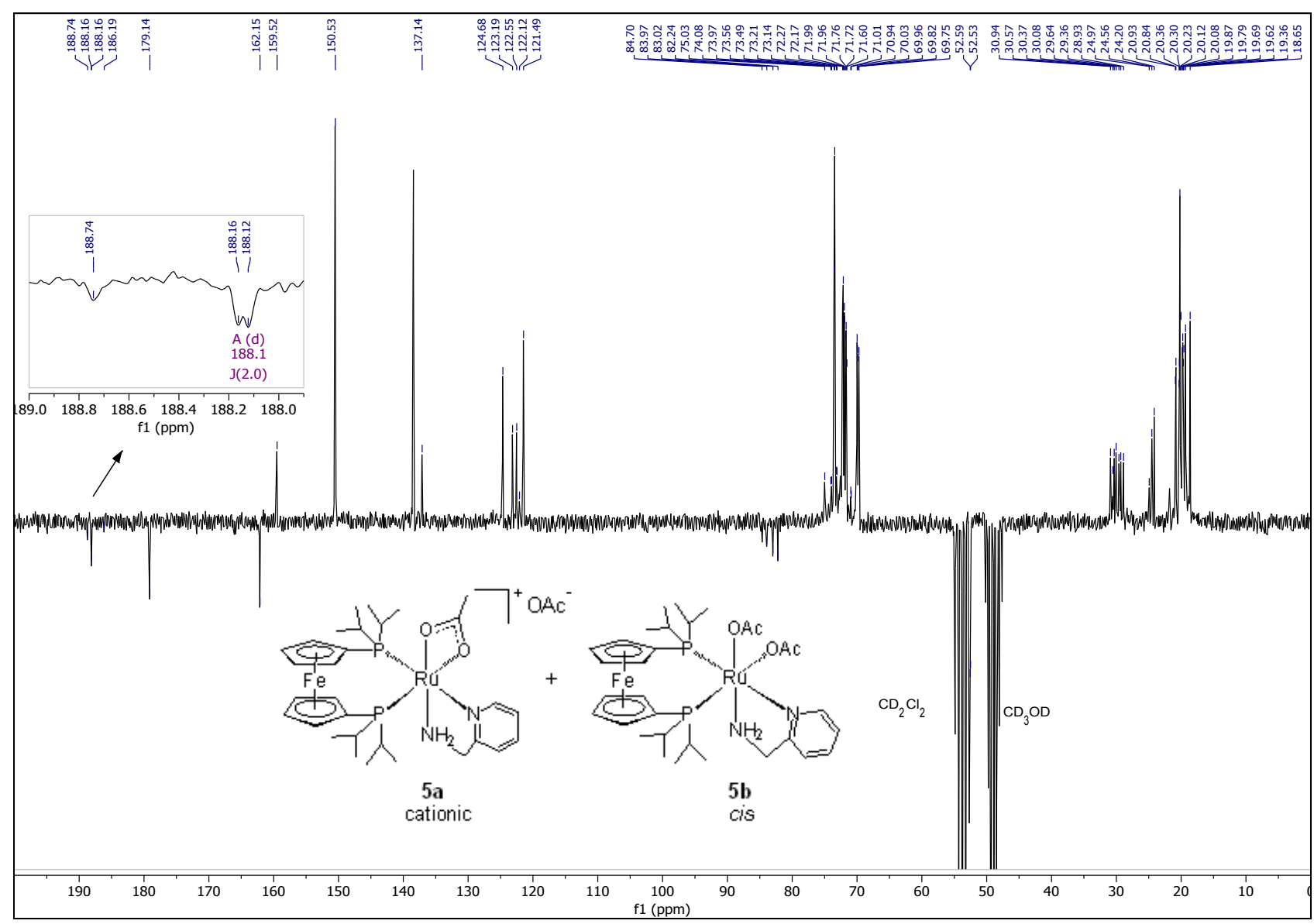

Figure S20. ${ }^{13} \mathrm{C}\left\{{ }^{1} \mathrm{H}\right\}$ PENDANT NMR spectrum $(50.3 \mathrm{MHz})$ of the mixture 4:1 of the cationic species $\left[\mathrm{Ru}\left(\eta^{2}-\mathrm{OAc}\right)(\mathrm{D} i \mathrm{PPF})(\operatorname{ampy})\right] \mathrm{OAc} \quad(\mathbf{5 a})$ and $c i s-\left[\mathrm{Ru}\left(\eta^{1}-\mathrm{OAc}\right)_{2}(\mathrm{D} i \mathrm{PPF})(\operatorname{ampy})\right]$ (5b) in $\mathrm{CD}_{2} \mathrm{Cl}_{2} / \mathrm{CD}_{3} \mathrm{OD}$ at $20{ }^{\circ} \mathrm{C}$. 


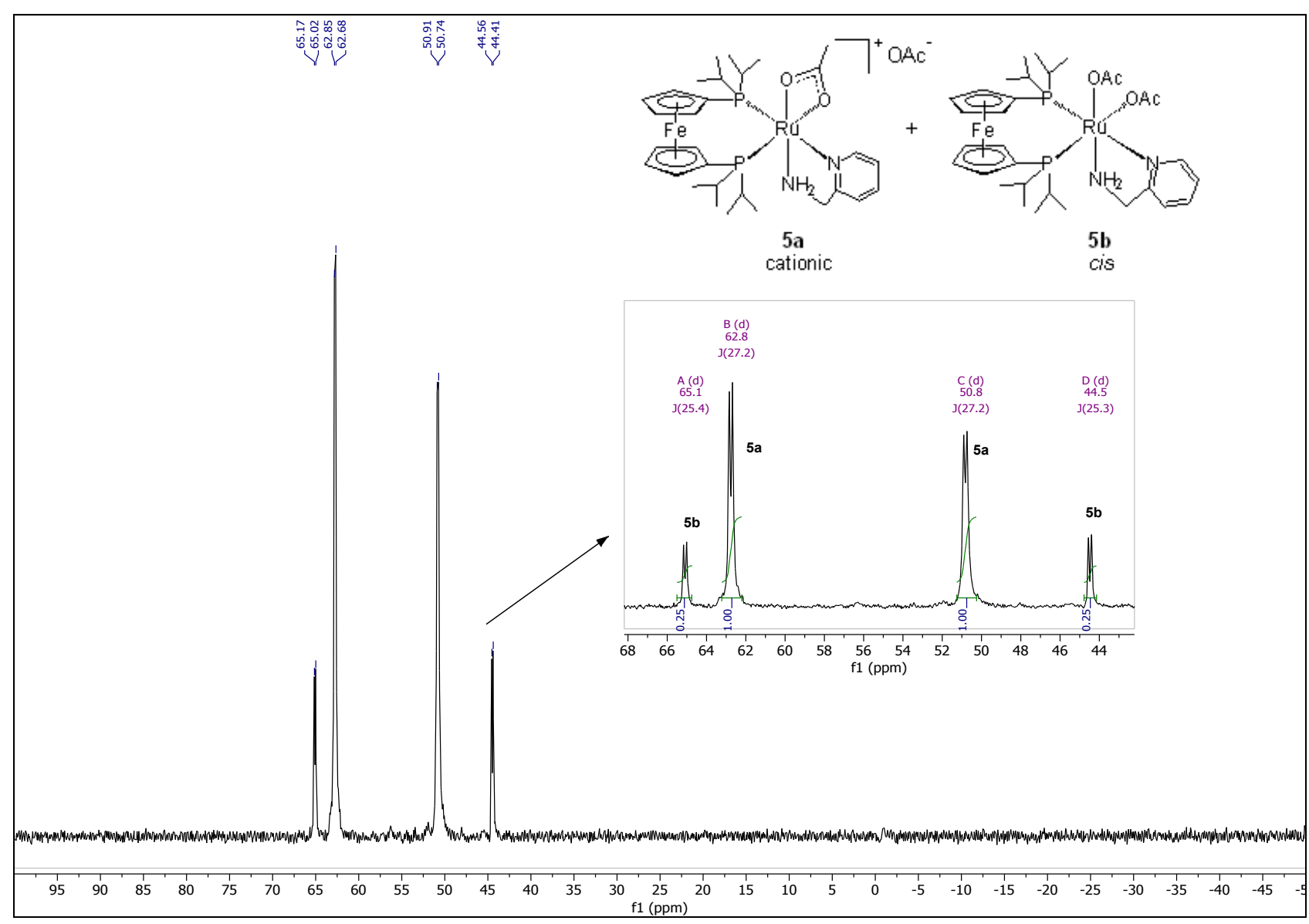

Figure S21. ${ }^{31} \mathrm{P}\left\{{ }^{1} \mathrm{H}\right\}$ NMR spectrum $(162.0 \mathrm{MHz})$ of the mixture $4: 1$ of the cationic species $\left[\mathrm{Ru}\left(\eta^{2}\right.\right.$ $\mathrm{OAc})(\mathrm{D} i \mathrm{PPF})(\mathrm{ampy})] \mathrm{OAc}(\mathbf{5 a})$ and $c i s-\left[\mathrm{Ru}\left(\eta^{1}-\mathrm{OAc}\right)_{2}(\mathrm{D} i \mathrm{PPF})(\mathrm{ampy})\right](\mathbf{5 b})$ in $\mathrm{CD}_{3} \mathrm{OD}$ at $25^{\circ} \mathrm{C}$. 


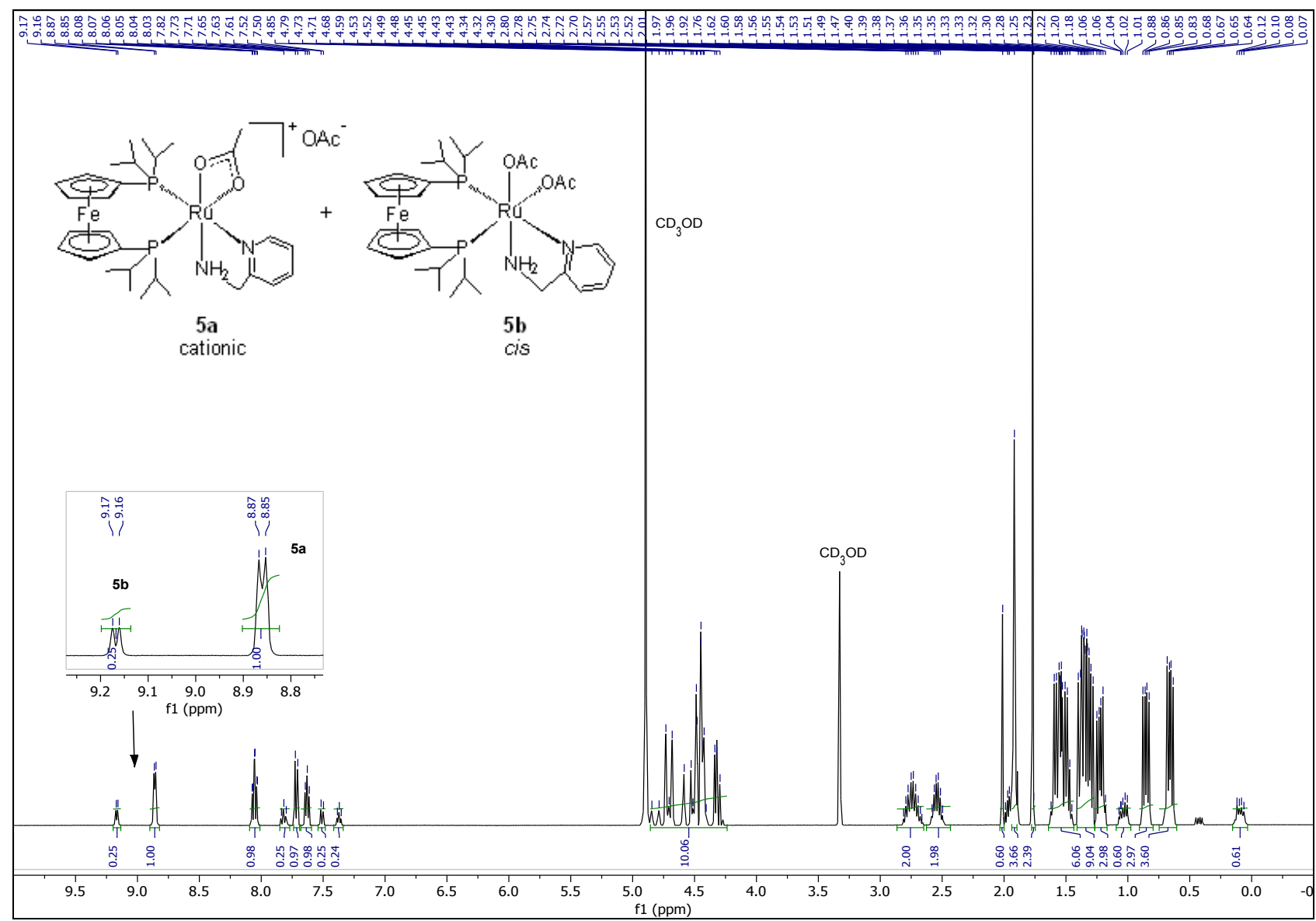

Figure S22. ${ }^{1} \mathrm{H}$ NMR spectrum $(400.1 \mathrm{MHz})$ of the mixture $4: 1$ of the cationic species $\left[\mathrm{Ru}\left(\eta^{2}\right.\right.$ $\mathrm{OAc})(\mathrm{D} i \mathrm{PPF})(\mathrm{ampy})] \mathrm{OAc}(\mathbf{5 a})$ and $c i s-\left[\mathrm{Ru}\left(\eta^{1}-\mathrm{OAc}\right)_{2}(\mathrm{D} i \mathrm{PPF})(\mathrm{ampy})\right](\mathbf{5 b})$ in $\mathrm{CD}_{3} \mathrm{OD}$ at $25^{\circ} \mathrm{C}$. 


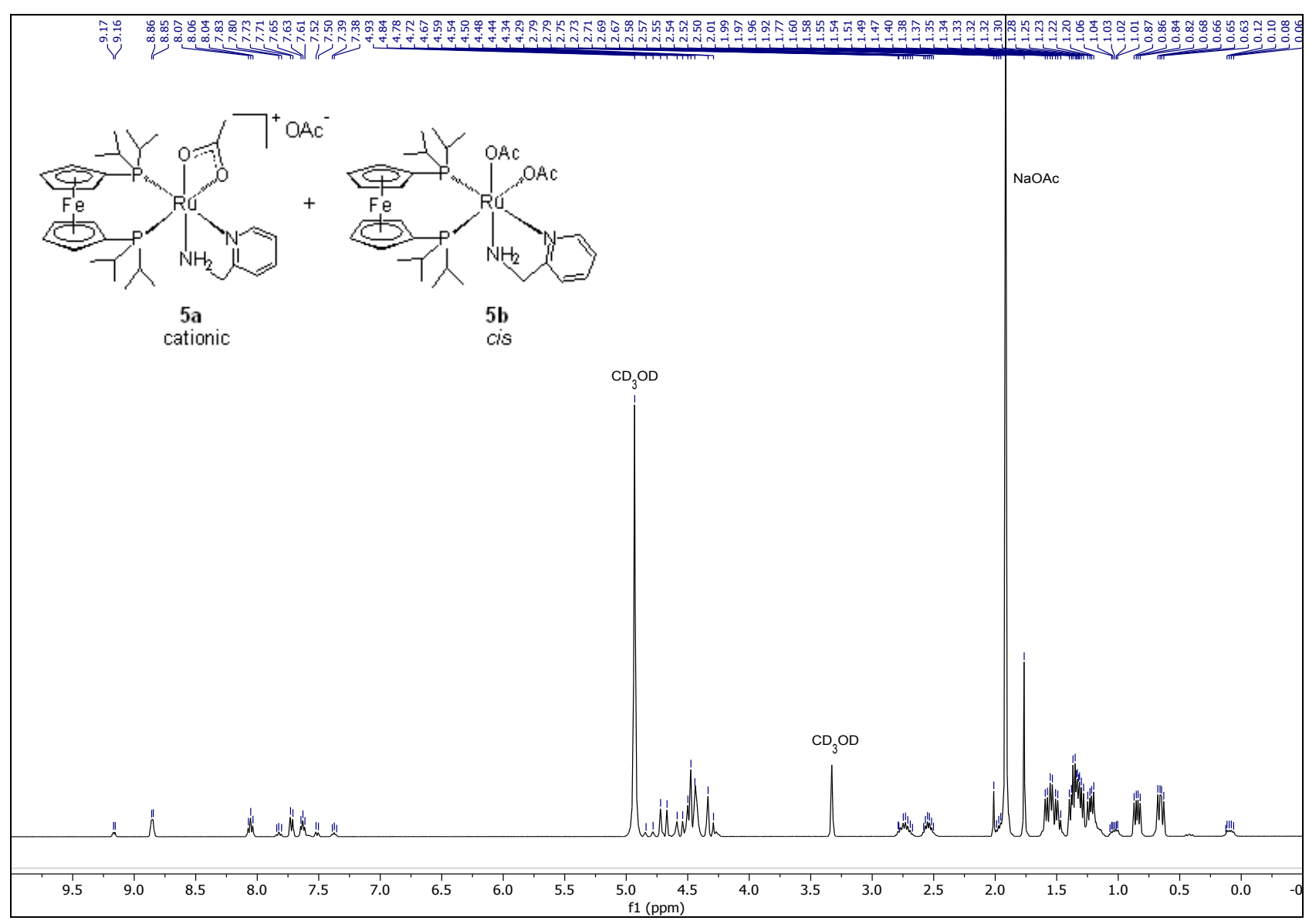

Figure S23. ${ }^{1} \mathrm{H}$ NMR spectrum $(400.1 \mathrm{MHz})$ of the mixture $4: 1$ of the cationic species $\left[\mathrm{Ru}\left(\eta^{2}\right.\right.$ $\mathrm{OAc})(\mathrm{D} i \mathrm{PPF})(\mathrm{ampy})] \mathrm{OAc}(\mathbf{5 a})$ and $c i s-\left[\mathrm{Ru}\left(\eta^{1}-\mathrm{OAc}\right)_{2}(\mathrm{D} i \mathrm{PPF})(\mathrm{ampy})\right](\mathbf{5 b})$ after the addition of $\mathrm{NaOAc}$ (3.5 equiv) in $\mathrm{CD}_{3} \mathrm{OD}$ at $25^{\circ} \mathrm{C}$. 


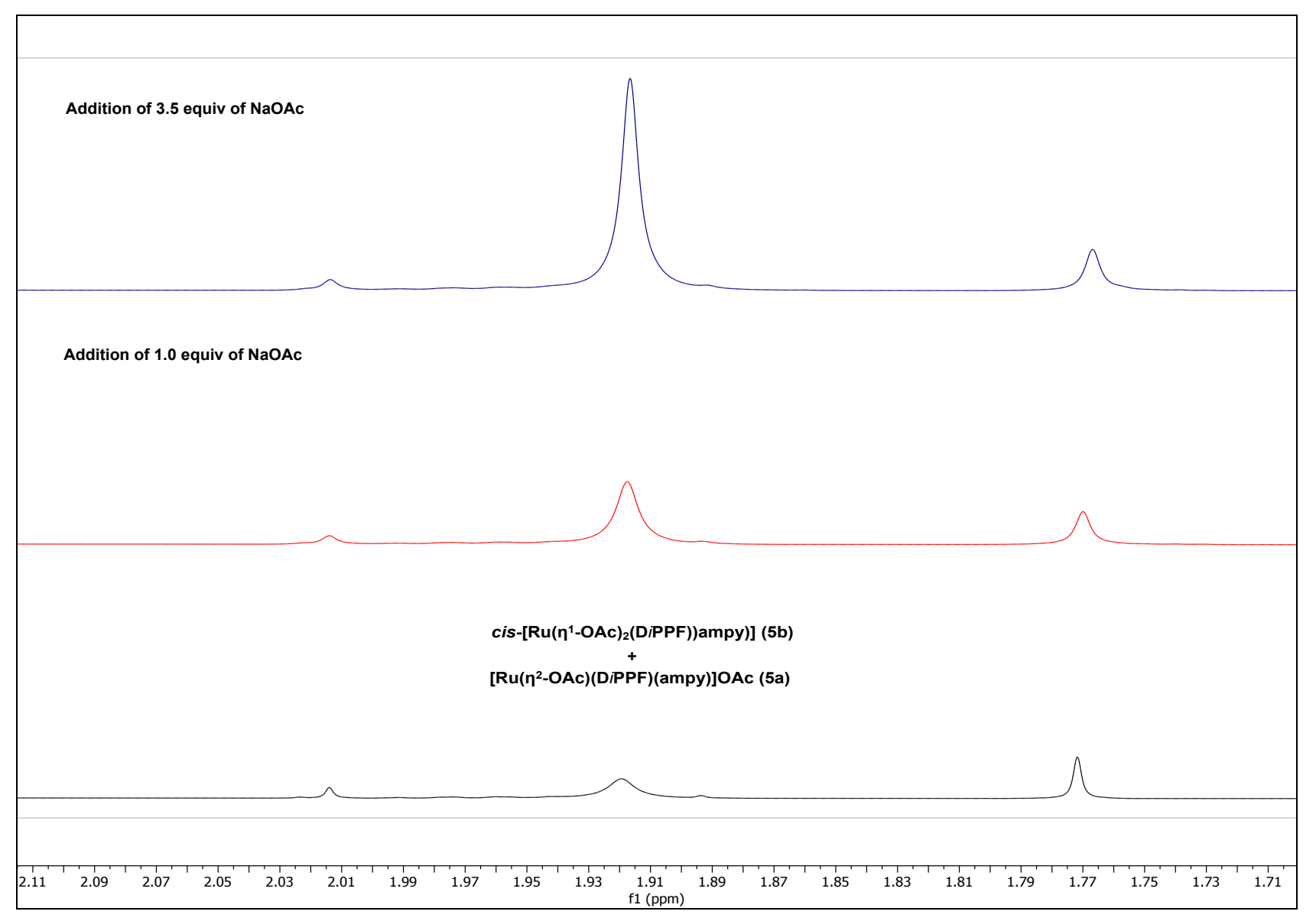

Figure S24. Effect of the addition of NaOAc (3.5 equiv) to the mixture 4:1 of the cationic species $\left[\mathrm{Ru}\left(\eta^{2}-\mathrm{OAc}\right)(\mathrm{D} i \mathrm{PPF})(\mathrm{ampy})\right] \mathrm{OAc}(\mathbf{5 a})$ and $c i s-\left[\mathrm{Ru}\left(\eta^{1}-\mathrm{OAc}\right)_{2}(\mathrm{D} i \mathrm{PPF})(\mathrm{ampy})\right](\mathbf{5 b})$ in the methyl acetate region of the ${ }^{1} \mathrm{H}$ NMR spectrum $(400.1 \mathrm{MHz})$ in $\mathrm{CD}_{3} \mathrm{OD}$ at $25^{\circ} \mathrm{C}$. 


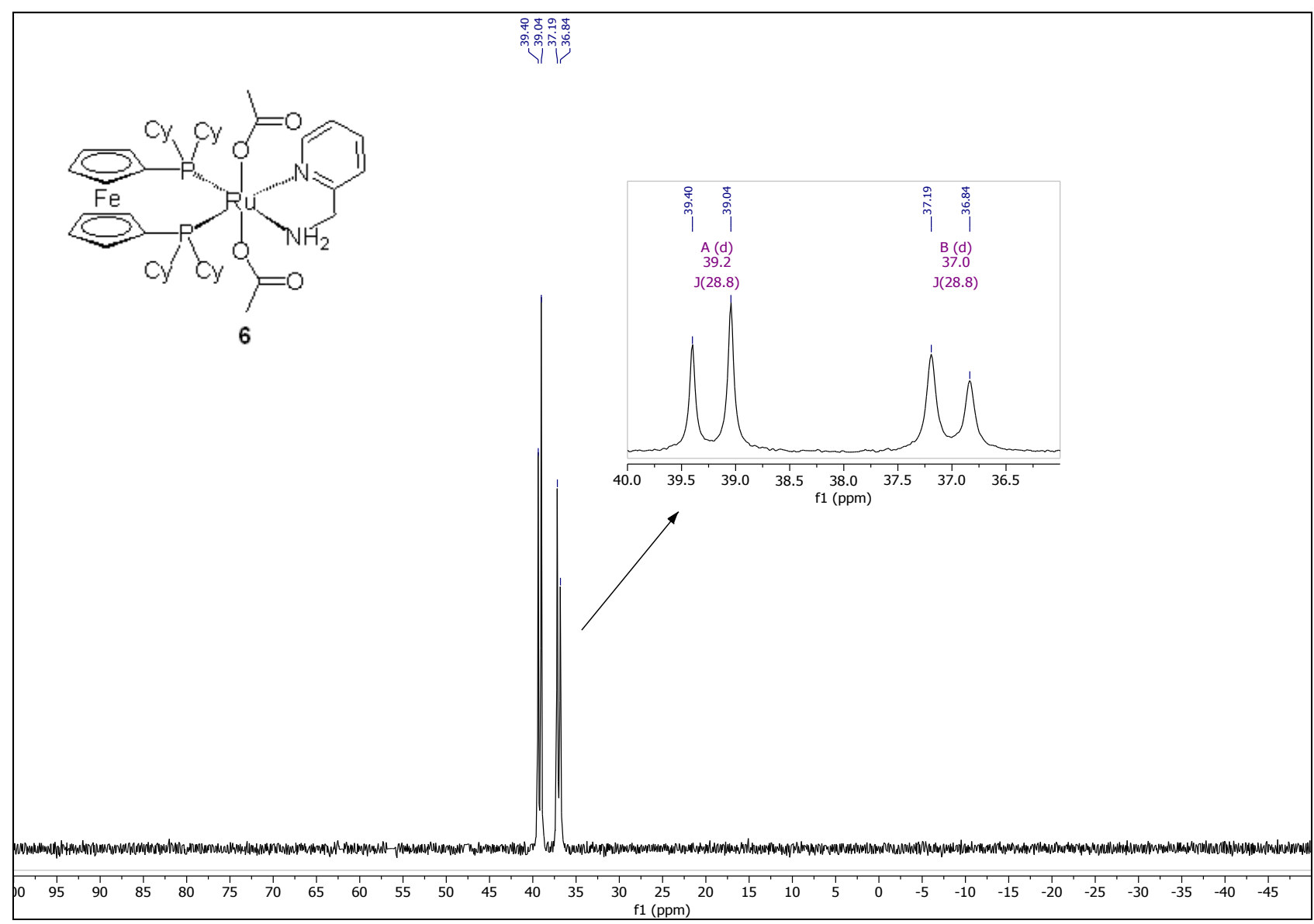

Figure S25. ${ }^{31} \mathrm{P}\left\{{ }^{1} \mathrm{H}\right\}$ NMR spectrum $(81.0 \mathrm{MHz})$ of trans-[Ru( $\left.\left.\eta^{1}-\mathrm{OAc}\right)_{2}(\mathrm{DCyPF})(\operatorname{ampy})\right](6)$ in $\mathrm{CDCl}_{3}$ at $20^{\circ} \mathrm{C}$. 


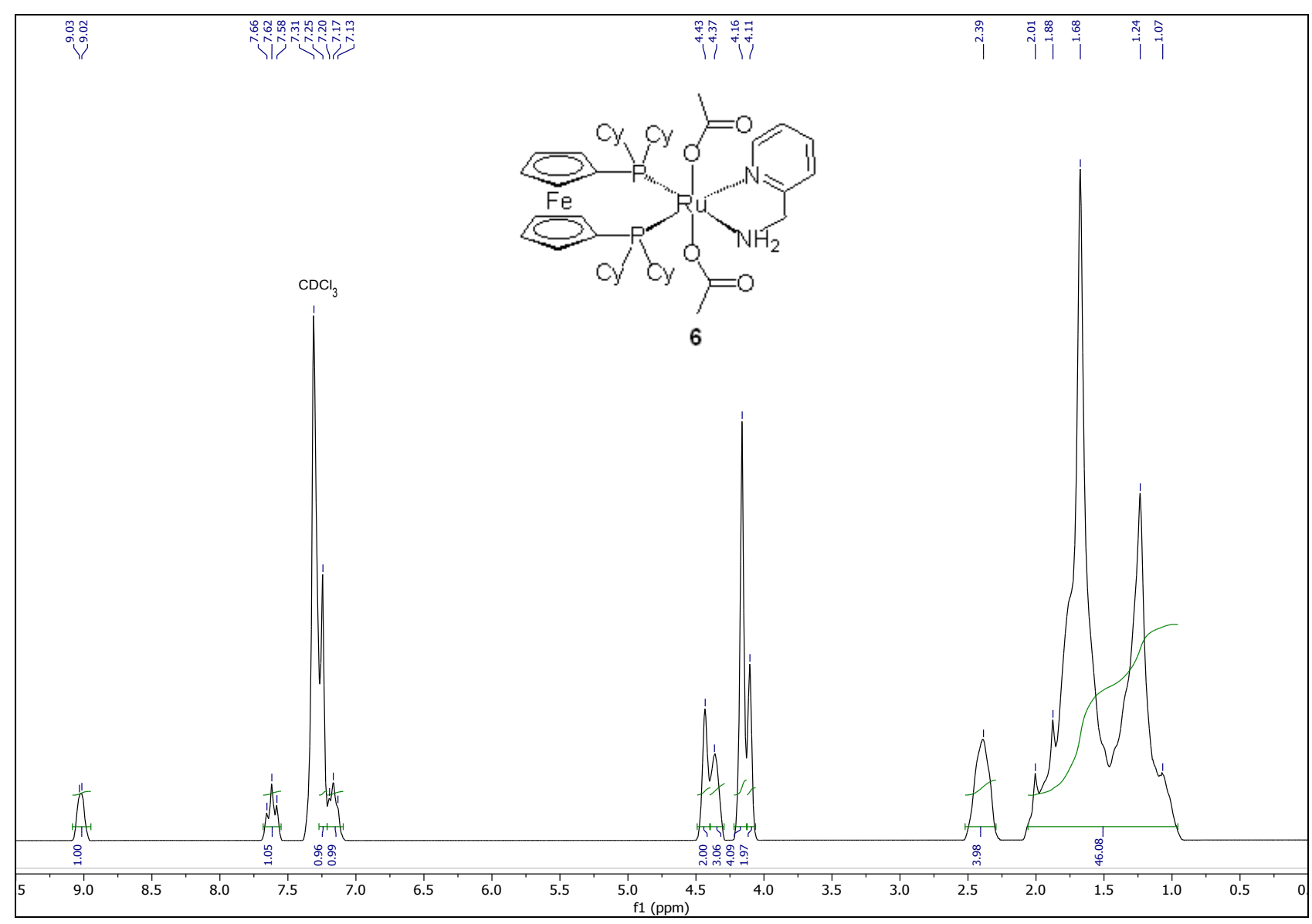

Figure S26. ${ }^{1} \mathrm{H}$ NMR spectrum $(200.1 \mathrm{MHz})$ of trans-[Ru( $\left.\left.\eta^{1}-\mathrm{OAc}\right)_{2}(\mathrm{DCyPF})(\mathrm{ampy})\right](\mathbf{6})$ in $\mathrm{CDCl}_{3}$ at $20^{\circ} \mathrm{C}$. 


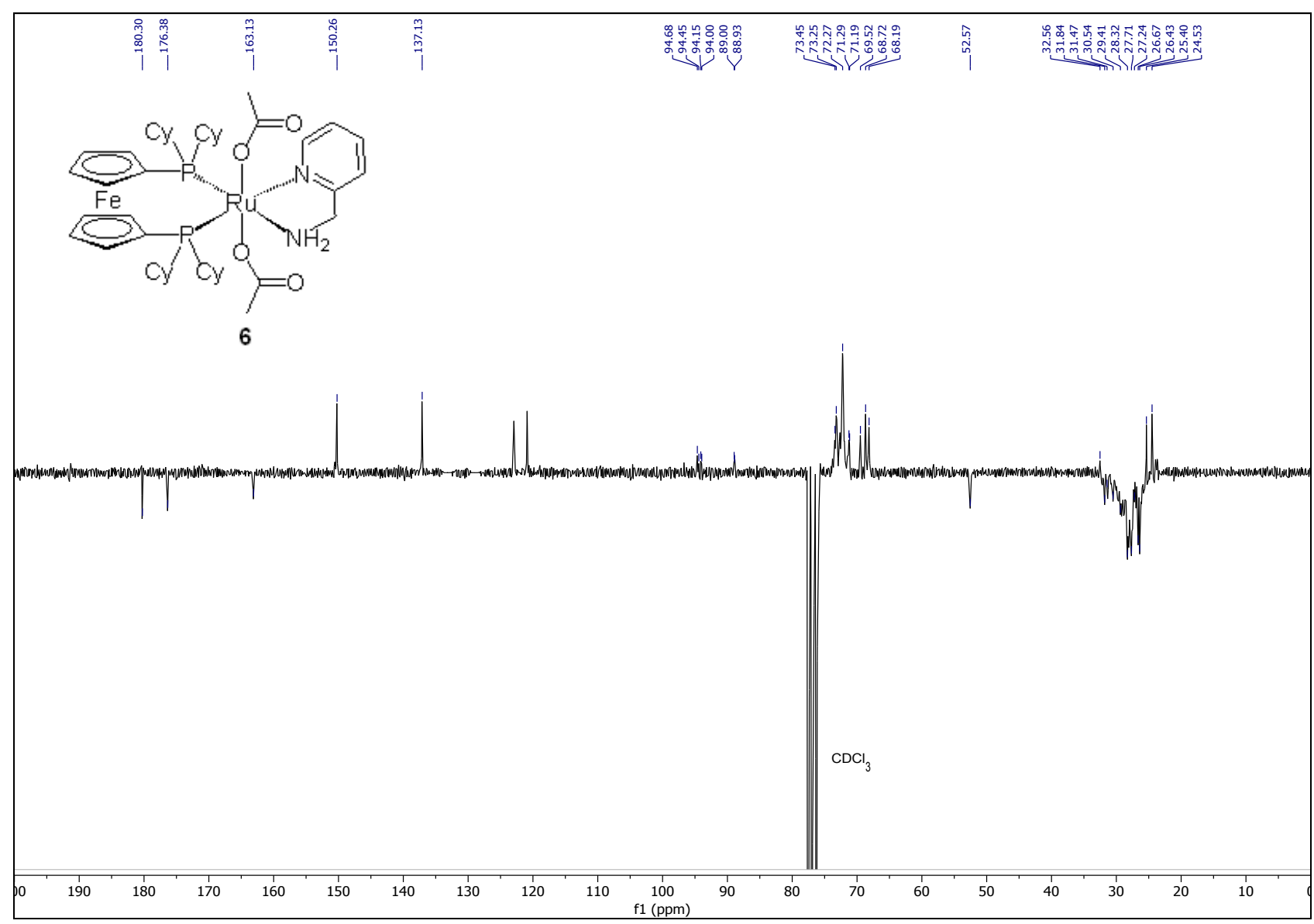

Figure S27. ${ }^{13} \mathrm{C}\left\{{ }^{1} \mathrm{H}\right\} \quad$ PENDANT $\quad$ NMR $\quad$ spectrum $\quad(50.3 \quad \mathrm{MHz})$ of trans-[Ru( $\left(\eta^{1}-\right.$ $\mathrm{OAc})_{2}(\mathrm{DCyPF})($ ampy) $](6)$ in $\mathrm{CDCl}_{3}$ at $20^{\circ} \mathrm{C}$. 


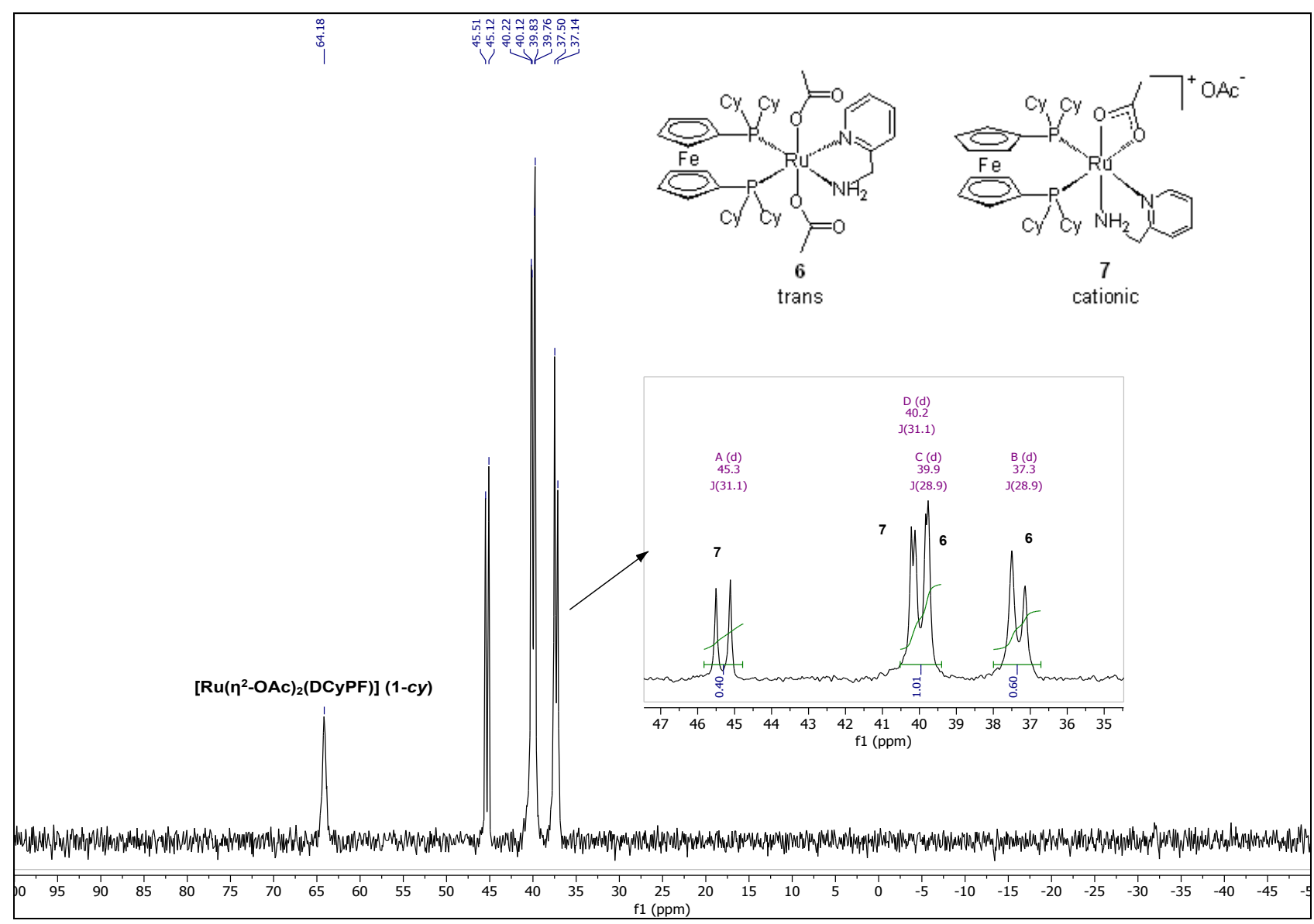

Figure S28. ${ }^{31} \mathrm{P}\left\{{ }^{1} \mathrm{H}\right\} \quad \mathrm{NMR}$ spectrum $(81.0 \mathrm{MHz})$ of the mixture of trans- $\left[\mathrm{Ru}\left(\eta^{1}-\right.\right.$ $\left.\mathrm{OAc}_{2}(\mathrm{DCyPF})(\mathrm{ampy})\right](6)$ and the cationic species $\left[\mathrm{Ru}\left(\eta^{2}-\mathrm{OAc}\right)(\mathrm{DCyPF})(\operatorname{ampy})\right] \mathrm{OAc}(7)$ (in a 3:2 ratio) in $\mathrm{CDCl}_{3}$ after $24 \mathrm{~h}$ at $\mathrm{RT}$. 


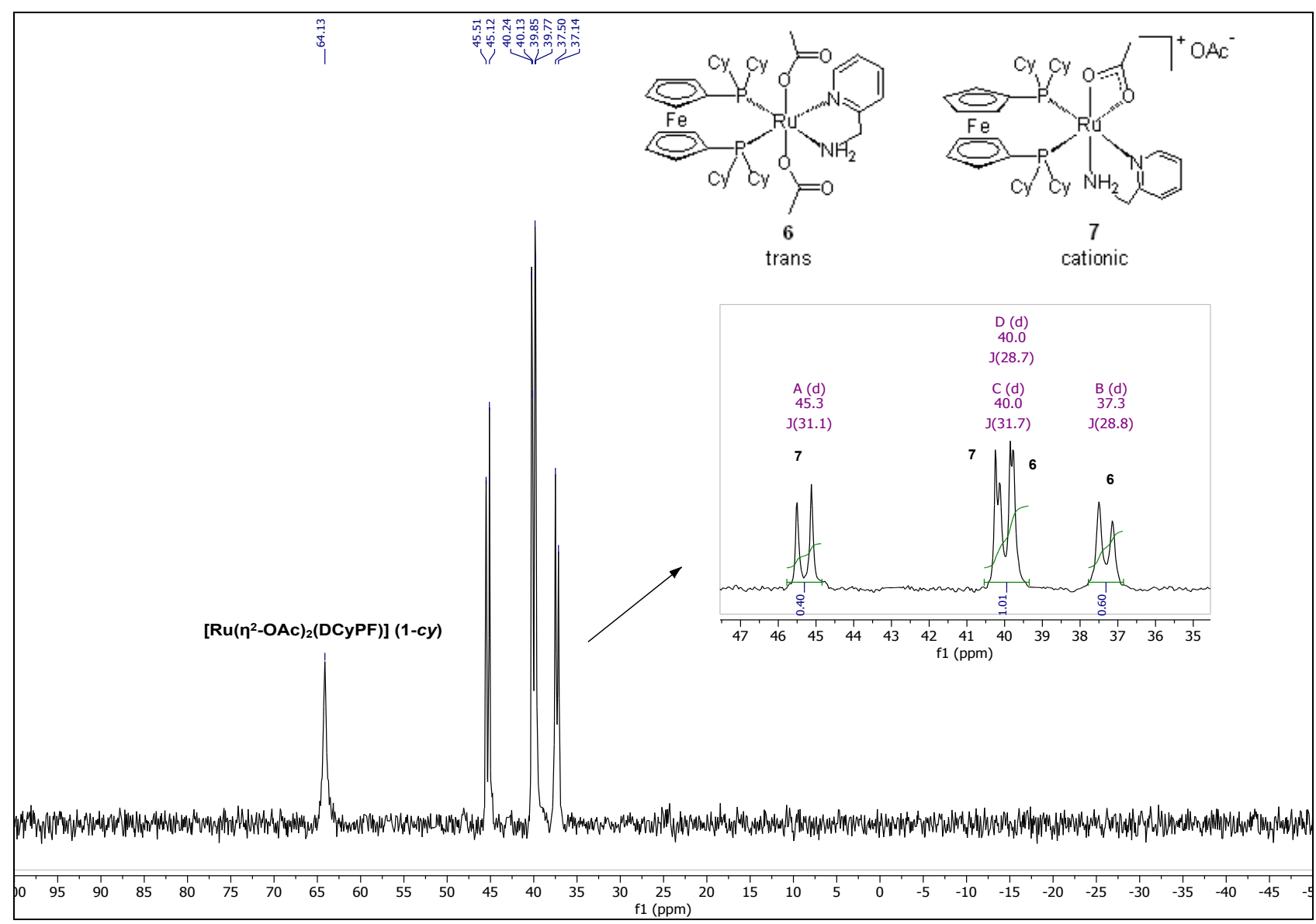

Figure S29. ${ }^{31} \mathrm{P}\left\{{ }^{1} \mathrm{H}\right\} \quad \mathrm{NMR}$ spectrum $(81.0 \mathrm{MHz})$ of the mixture of trans- $\left[\mathrm{Ru}\left(\eta^{1}-\right.\right.$ $\left.\mathrm{OAc})_{2}(\mathrm{DCyPF})(\mathrm{ampy})\right](\mathbf{6})$ and the cationic species $\left[\mathrm{Ru}\left(\eta^{2}-\mathrm{OAc}\right)(\mathrm{DCyPF})(\operatorname{ampy})\right] \mathrm{OAc}(7)$ (in a 3:2 ratio) in toluene- $d^{8}$ after $24 \mathrm{~h}$ at RT. 


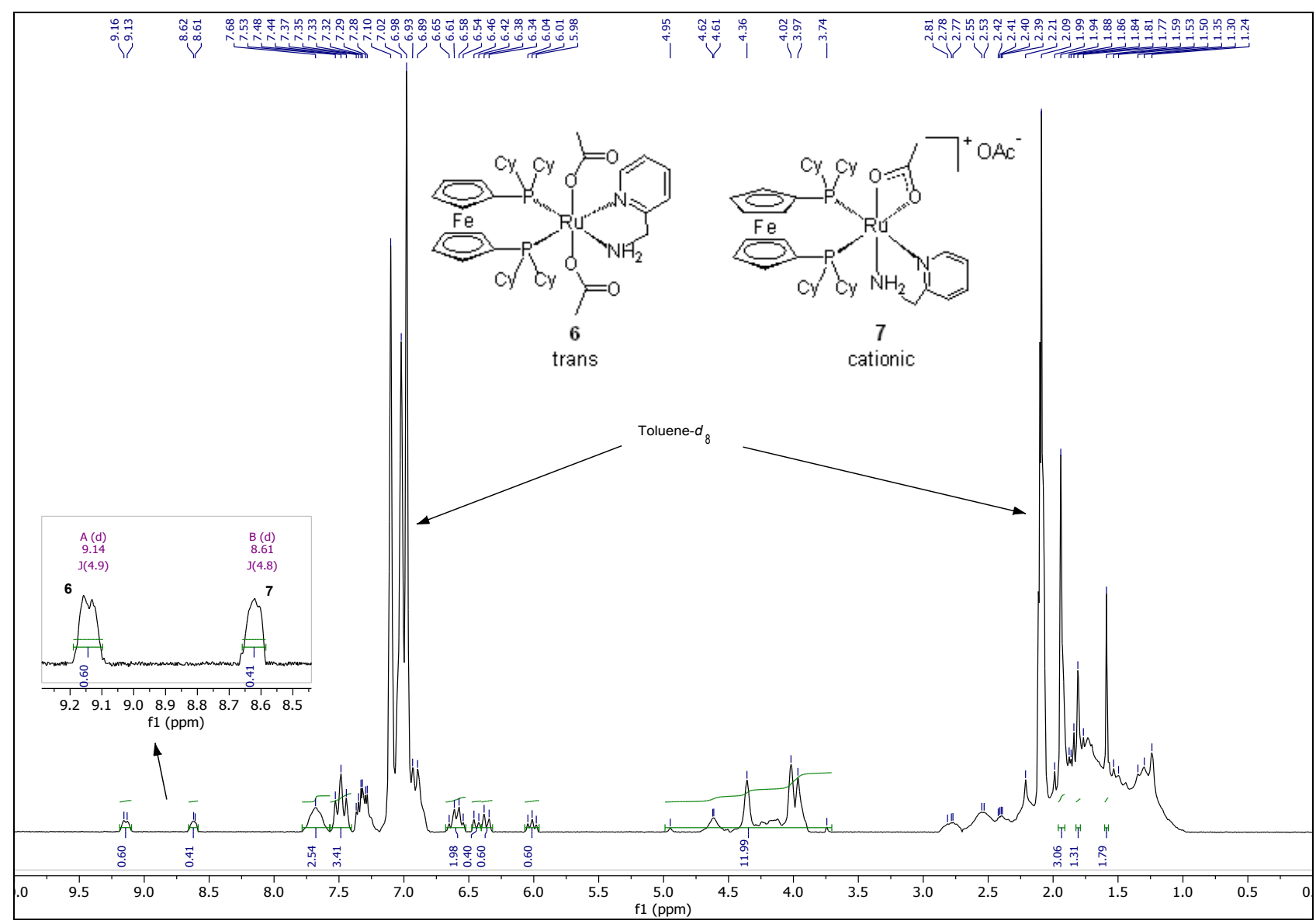

Figure S30. ${ }^{1} \mathrm{H}$ NMR spectrum $(200.1 \mathrm{MHz})$ of the mixture of trans-[Ru( $\left.\left.\eta^{1}-\mathrm{OAc}\right)_{2}(\mathrm{DCyPF})(\operatorname{ampy})\right]$ (6) and the cationic species $\left[\mathrm{Ru}\left(\eta^{2}-\mathrm{OAc}\right)(\mathrm{DCyPF})(\operatorname{ampy})\right] \mathrm{OAc}(7)$ (in a 3:2 ratio) in toluene- $d^{8}$ after $24 \mathrm{~h}$ at RT. 


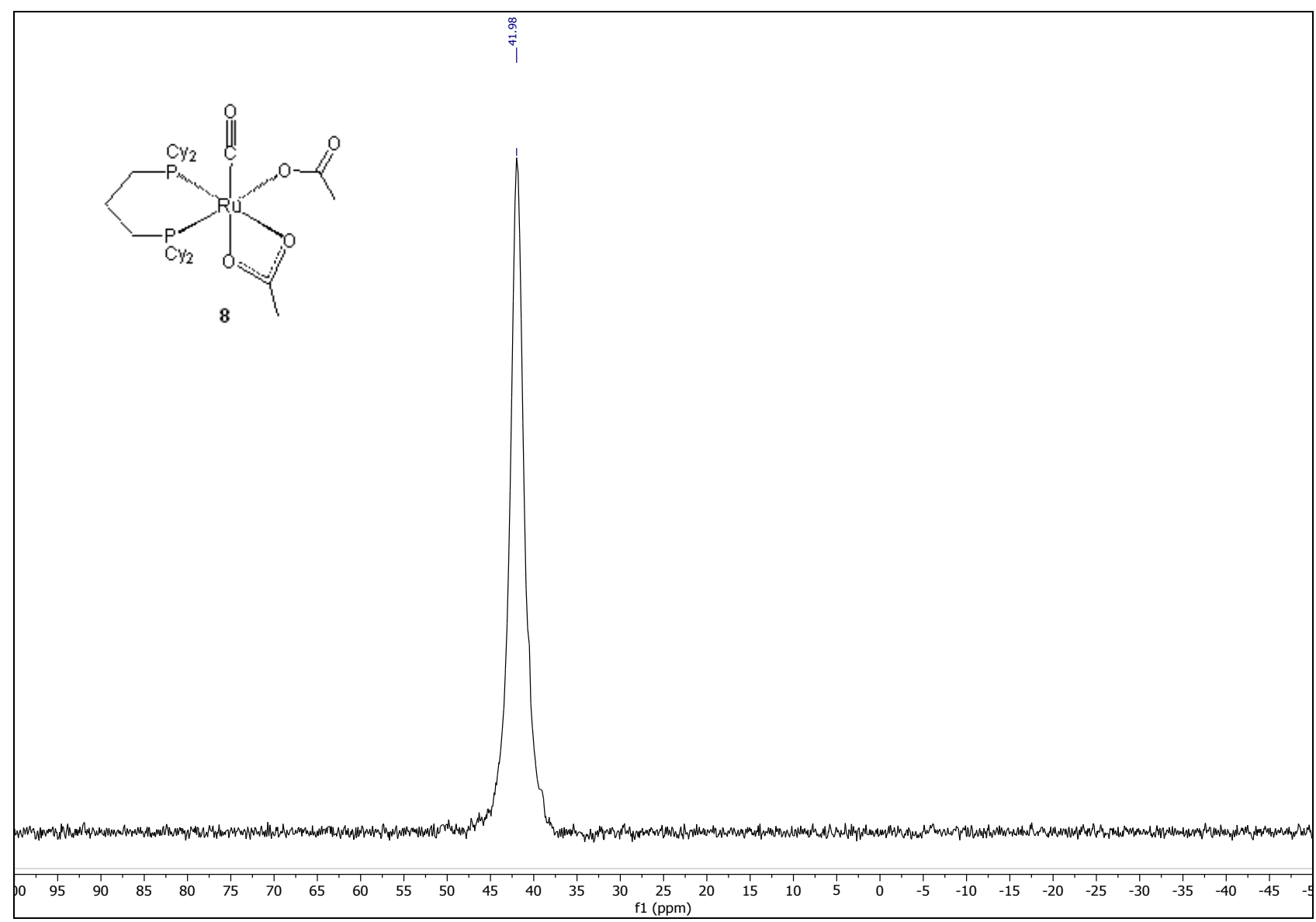

Figure S31. ${ }^{31} \mathrm{P}\left\{{ }^{1} \mathrm{H}\right\}$ NMR spectrum $(162.0 \mathrm{MHz})$ of $\left[\mathrm{Ru}\left(\eta^{1}-\mathrm{OAc}\right)\left(\eta^{2}-\mathrm{OAc}\right)(\mathrm{DCyPP})(\mathrm{CO})\right](\mathbf{8})$ in $\mathrm{CD}_{2} \mathrm{Cl}_{2}$ at $25^{\circ} \mathrm{C}$. 


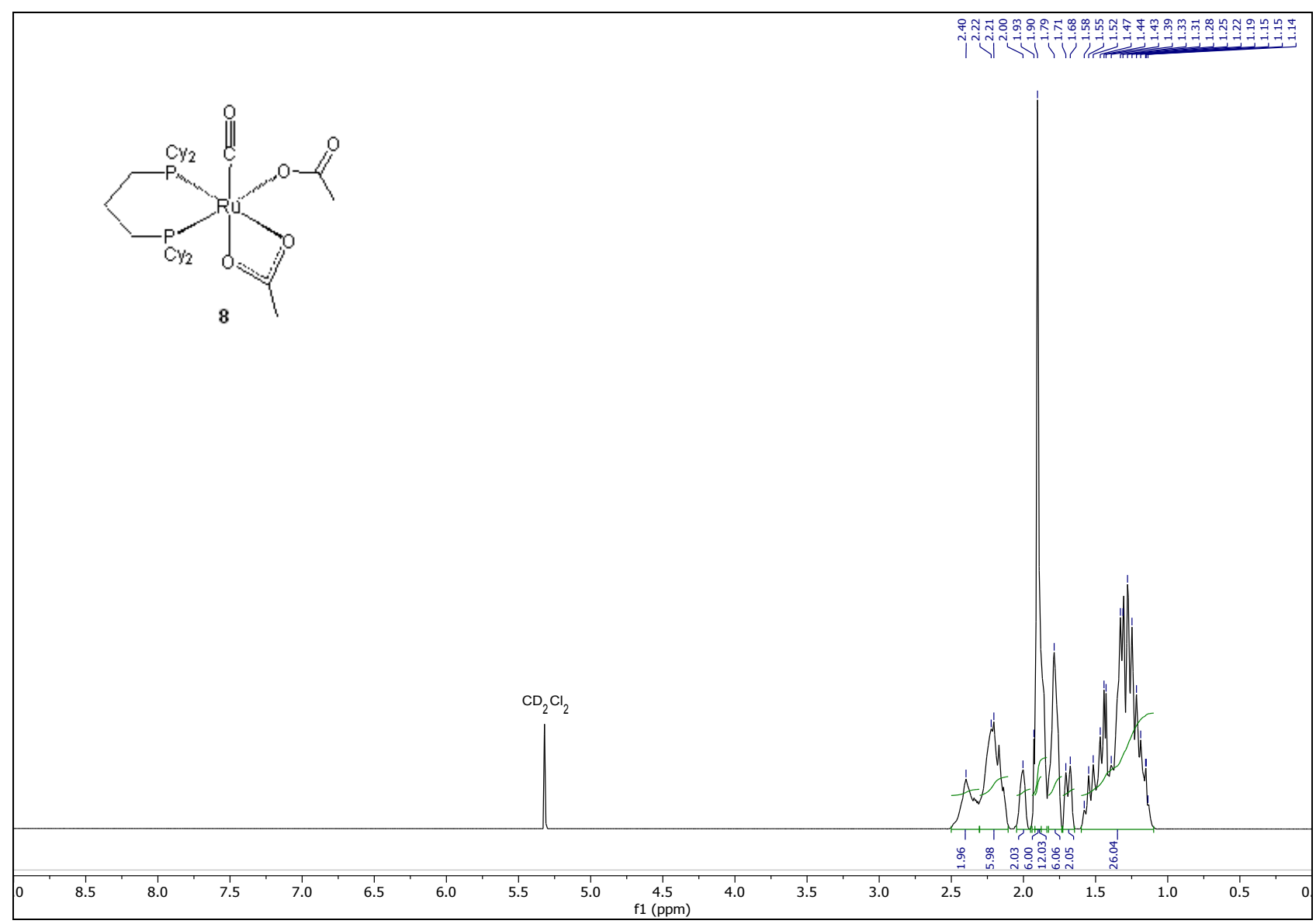

Figure S32. ${ }^{1} \mathrm{H}$ NMR spectrum $(400.1 \mathrm{MHz})$ of $\left[\mathrm{Ru}\left(\eta^{1}-\mathrm{OAc}\right)\left(\eta^{2}-\mathrm{OAc}\right)(\mathrm{DCyPP})(\mathrm{CO})\right](\mathbf{8})$ in $\mathrm{CD}_{2} \mathrm{Cl}_{2}$ at $25^{\circ} \mathrm{C}$. 


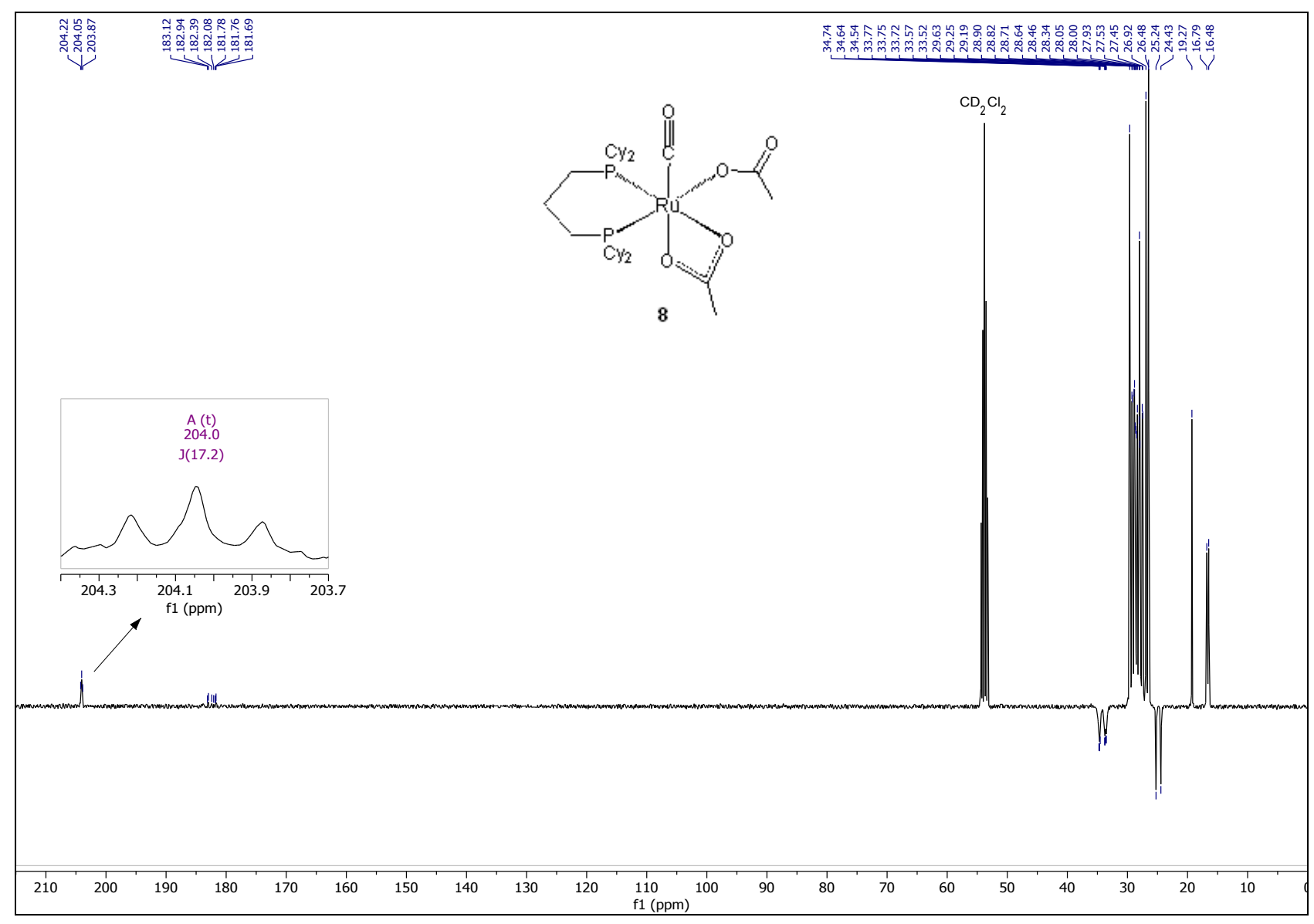

Figure S33. ${ }^{13} \mathrm{C}\left\{{ }^{1} \mathrm{H}\right\}$ DEPTQ NMR spectrum $(100.6 \mathrm{MHz})$ of $\left[\mathrm{Ru}\left(\eta^{1}-\mathrm{OAc}\right)\left(\eta^{2}-\mathrm{OAc}\right)(\mathrm{DCyPP})(\mathrm{CO})\right]$ (8) in $\mathrm{CD}_{2} \mathrm{Cl}_{2}$ at $25^{\circ} \mathrm{C}$. 


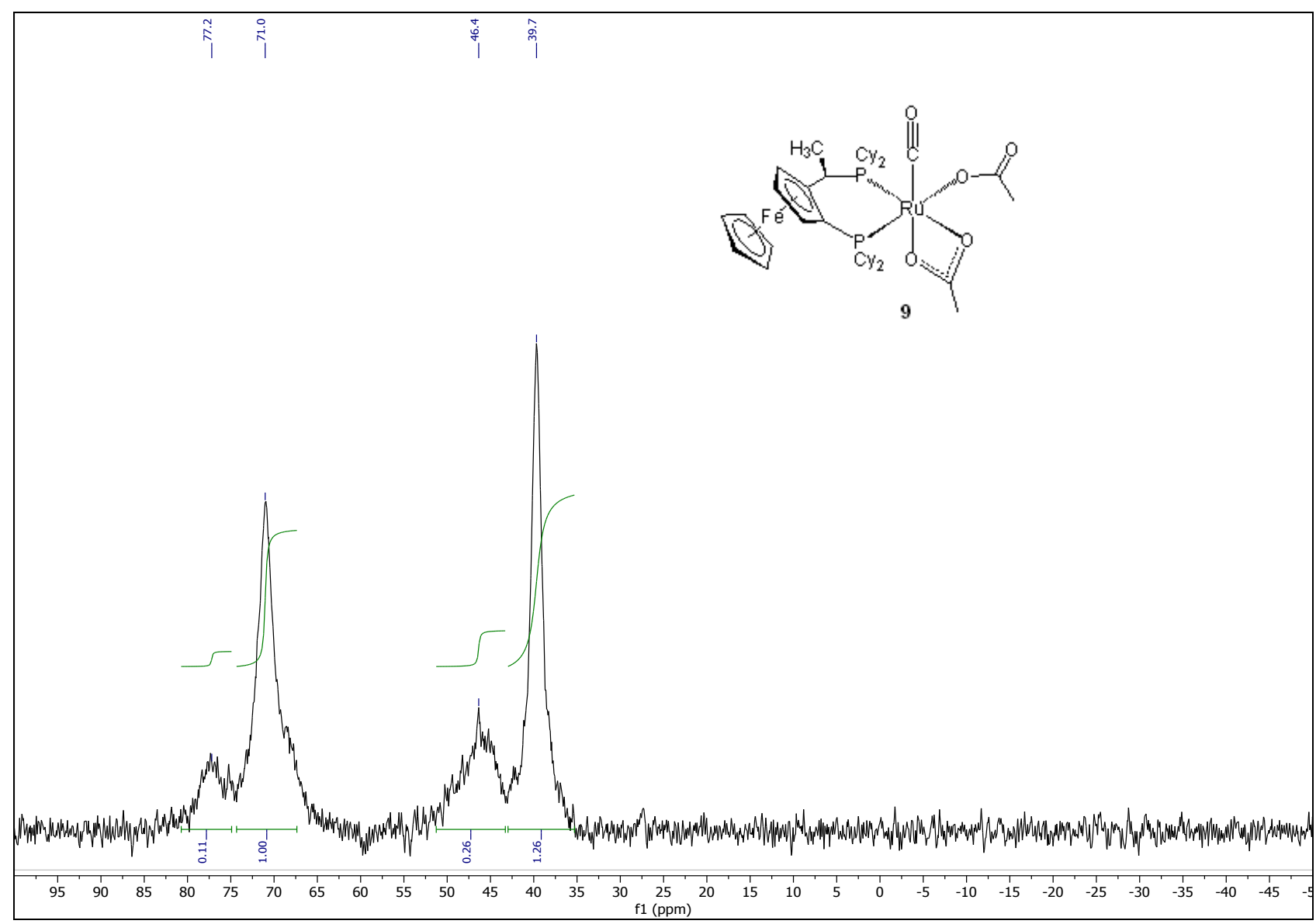

Figure S34. ${ }^{31} \mathrm{P}\left\{{ }^{1} \mathrm{H}\right\}$ NMR spectrum $(162.0 \mathrm{MHz})$ of $\left[\mathrm{Ru}\left(\eta^{1}-\mathrm{OAc}\right)\left(\eta^{2}-\mathrm{OAc}\right)\left(\mathrm{Josiphos}{ }^{\mathrm{cy}}\right)(\mathrm{CO})\right](9)$ in $\mathrm{CD}_{2} \mathrm{Cl}_{2}$ at $20{ }^{\circ} \mathrm{C}$. 


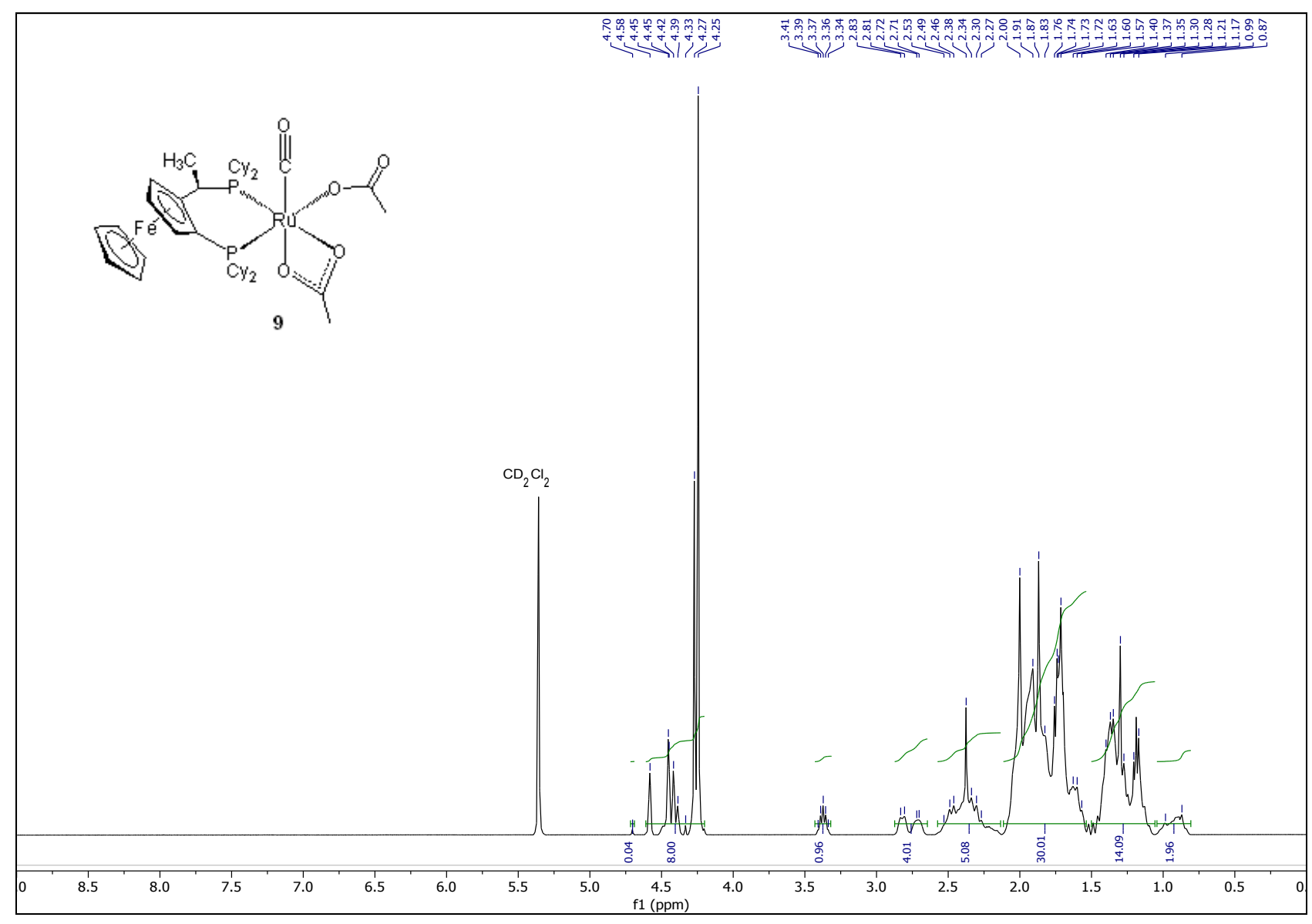

Figure S35. ${ }^{1} \mathrm{H}$ NMR spectrum $(400.1 \mathrm{MHz})$ of $\left[\mathrm{Ru}\left(\eta^{1}-\mathrm{OAc}\right)\left(\eta^{2}-\mathrm{OAc}\right)\left(\mathrm{Josiphos}^{\mathrm{cy}}\right)(\mathrm{CO})\right](9)$ in $\mathrm{CD}_{2} \mathrm{Cl}_{2}$ at $20^{\circ} \mathrm{C}$. 


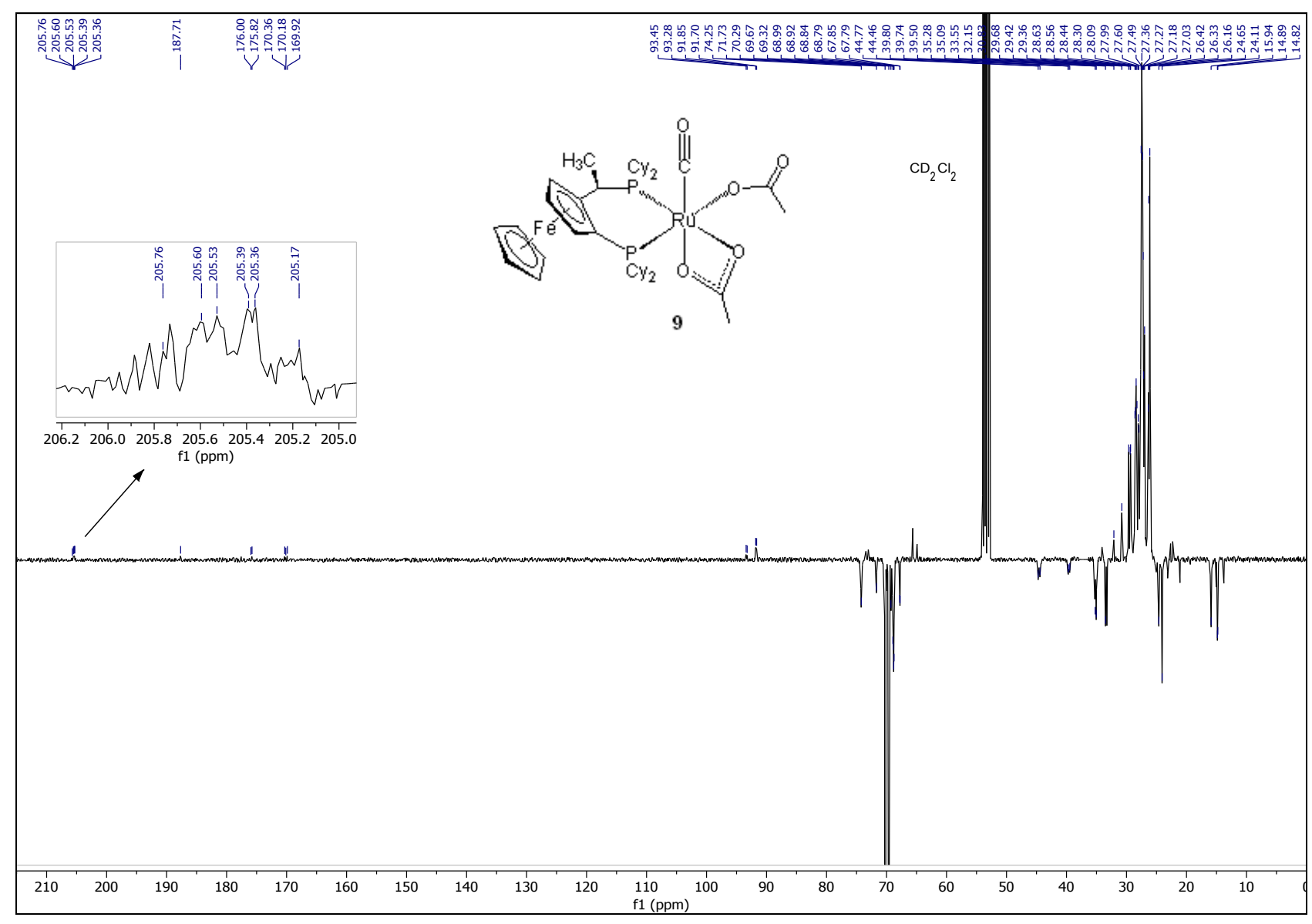

Figure S36. ${ }^{13} \mathrm{C}\left\{{ }^{1} \mathrm{H}\right\}$ DEPTQ NMR spectrum $(100.6 \mathrm{MHz})$ of $\left[\mathrm{Ru}\left(\eta^{1}-\mathrm{OAc}\right)\left(\eta^{2}-\right.\right.$ OAc)(Josiphos $\left.\left.{ }^{\mathrm{cy}}\right)(\mathrm{CO})\right](9)$ in $\mathrm{CD}_{2} \mathrm{Cl}_{2}$ at $20^{\circ} \mathrm{C}$. 


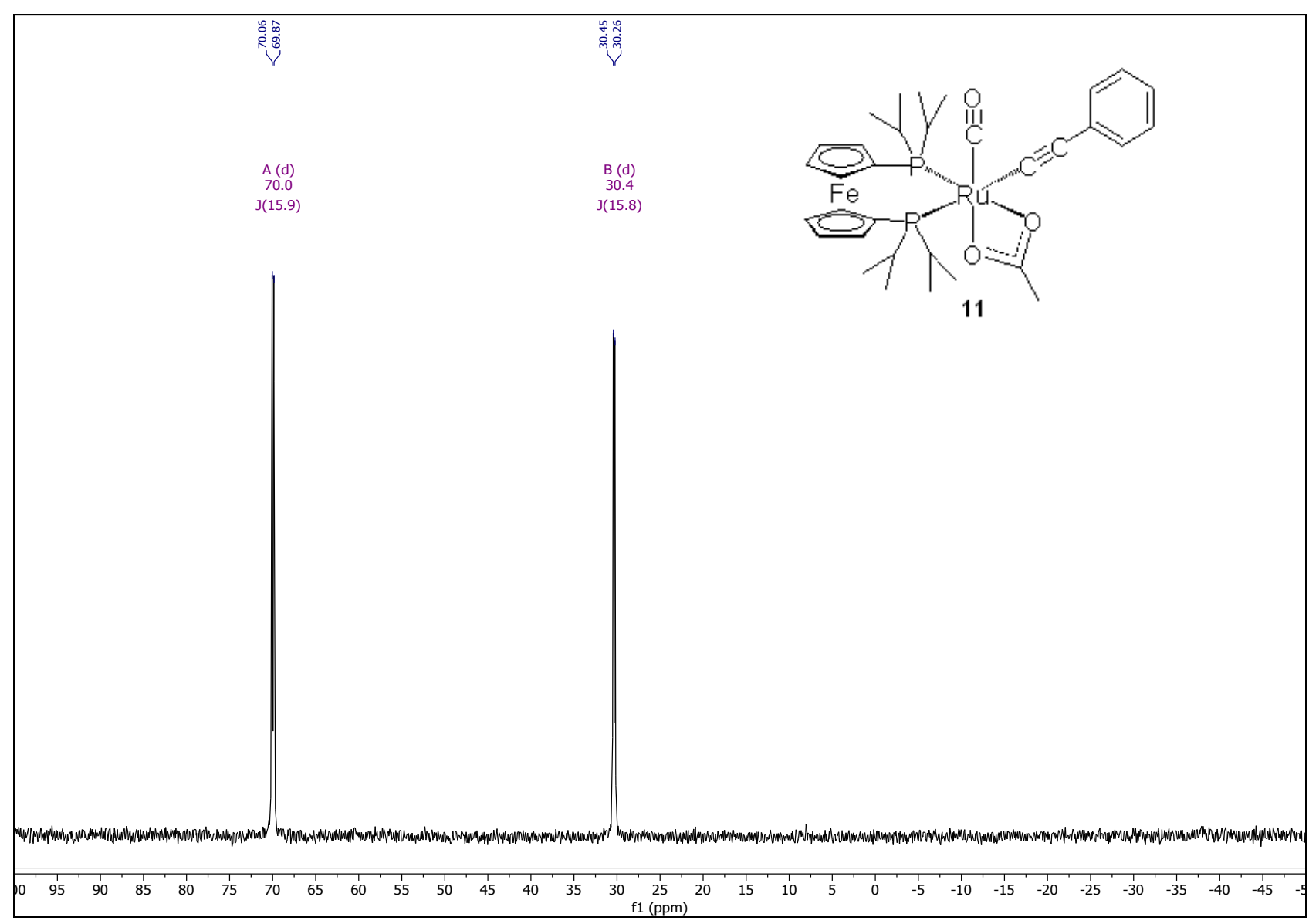

Figure S37. ${ }^{31} \mathrm{P}\left\{{ }^{1} \mathrm{H}\right\}$ NMR spectrum $(81.0 \mathrm{MHz})$ of $\left[\mathrm{Ru}\left(\eta^{2}-\mathrm{OAc}\right)(\mathrm{C} \equiv \mathrm{CPh})(\mathrm{D} i \mathrm{PPF})(\mathrm{CO})\right](\mathbf{1 1})$ in $\mathrm{CD}_{2} \mathrm{Cl}_{2}$ at $20{ }^{\circ} \mathrm{C}$. 


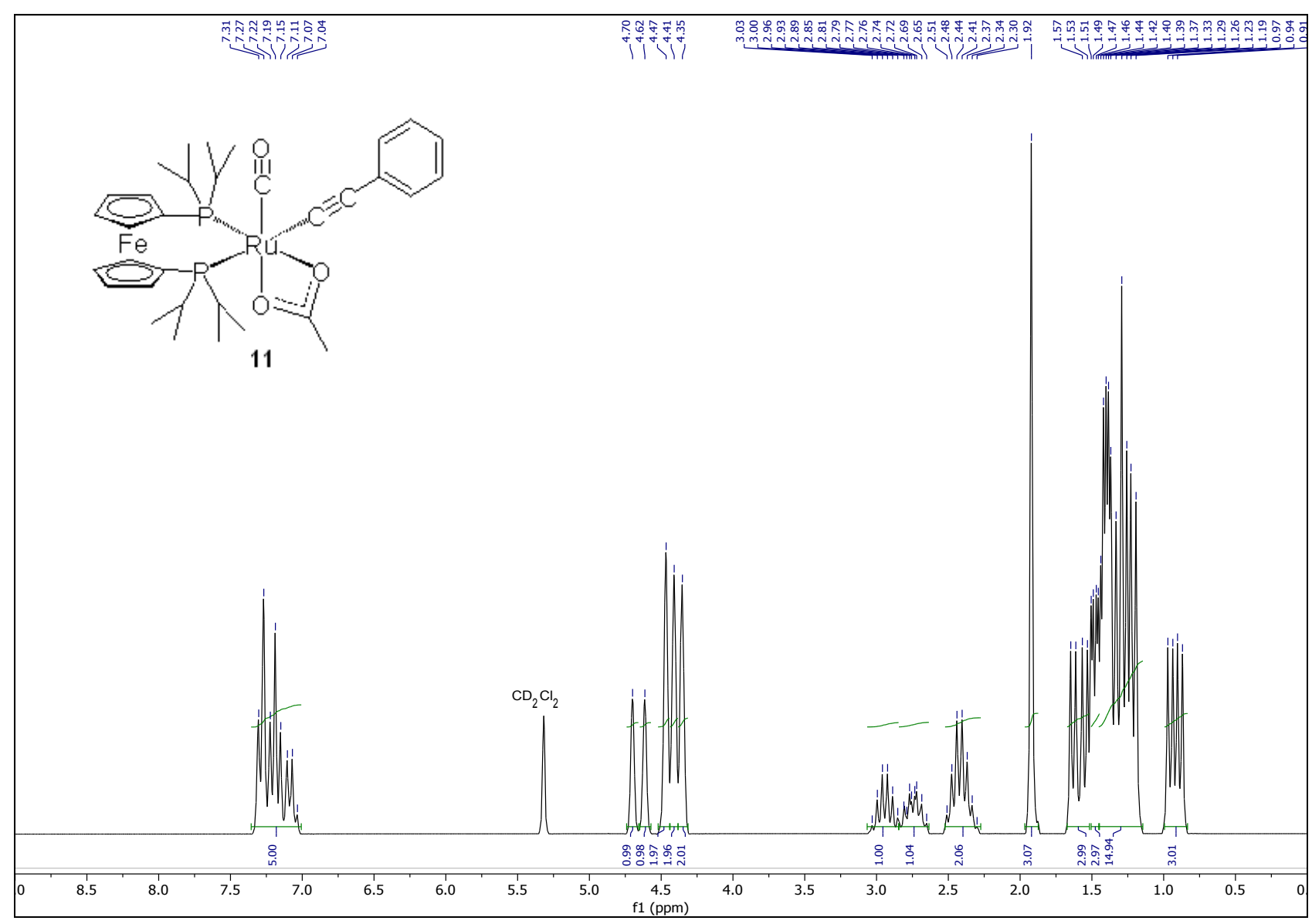

Figure S38. ${ }^{1} \mathrm{H}$ NMR spectrum $(200.1 \mathrm{MHz})$ of $\left[\mathrm{Ru}\left(\eta^{2}-\mathrm{OAc}\right)(\mathrm{C} \equiv \mathrm{CPh})(\mathrm{D} i \mathrm{PPF})(\mathrm{CO})\right](\mathbf{1 1})$ in $\mathrm{CD}_{2} \mathrm{Cl}_{2}$ at $20^{\circ} \mathrm{C}$. 


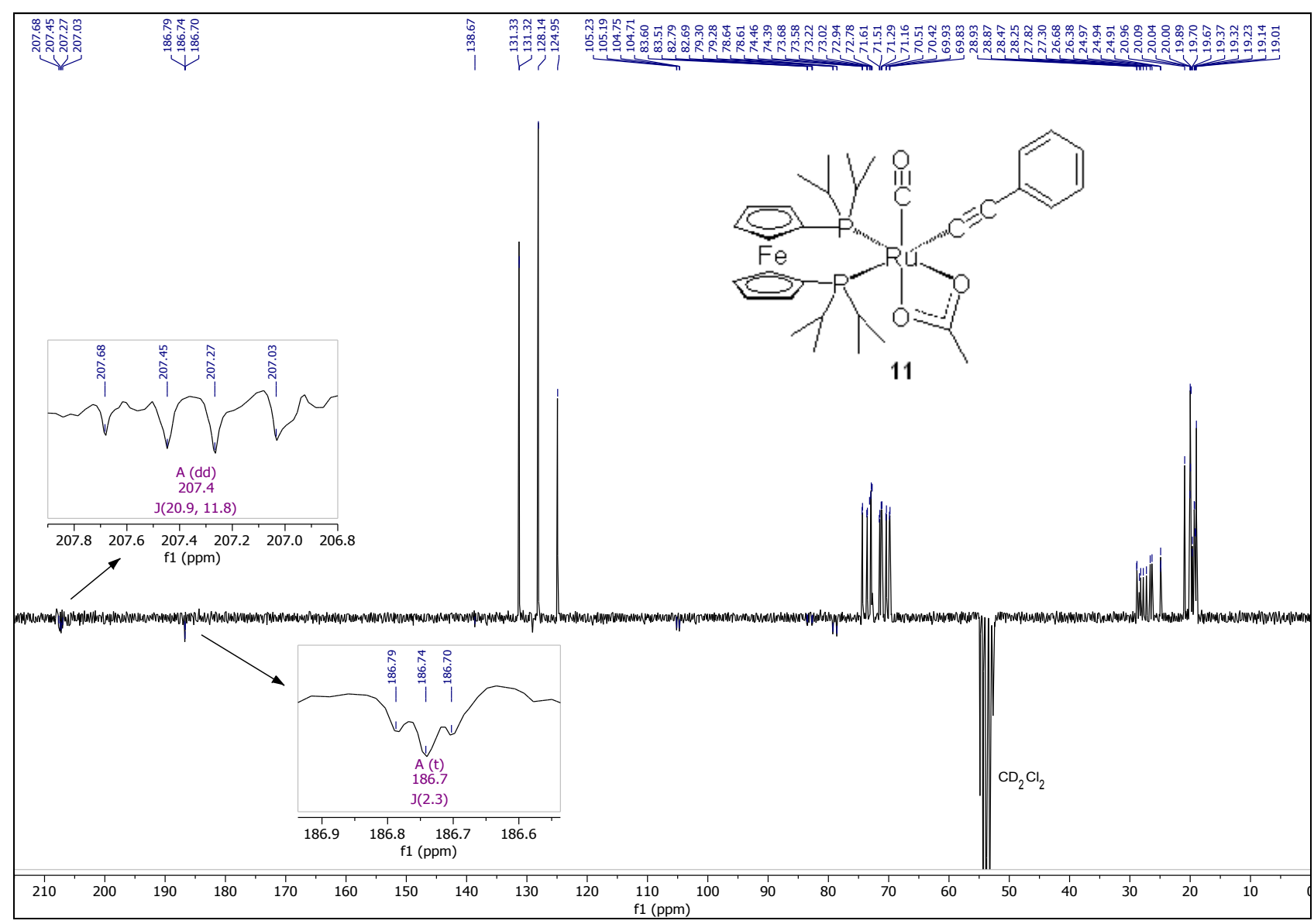

Figure S39. ${ }^{13} \mathrm{C}\left\{{ }^{1} \mathrm{H}\right\} \quad$ PENDANT $\quad \mathrm{NMR} \quad$ spectrum $\quad(50.3 \quad \mathrm{MHz}) \quad$ of $\quad\left[\mathrm{Ru}\left(\eta^{2}-\right.\right.$ $\mathrm{OAc})(\mathrm{C} \equiv \mathrm{CPh})(\mathrm{D} i \mathrm{PPF})(\mathrm{CO})](\mathbf{1 1})$ in $\mathrm{CD}_{2} \mathrm{Cl}_{2}$ at $20^{\circ} \mathrm{C}$. 


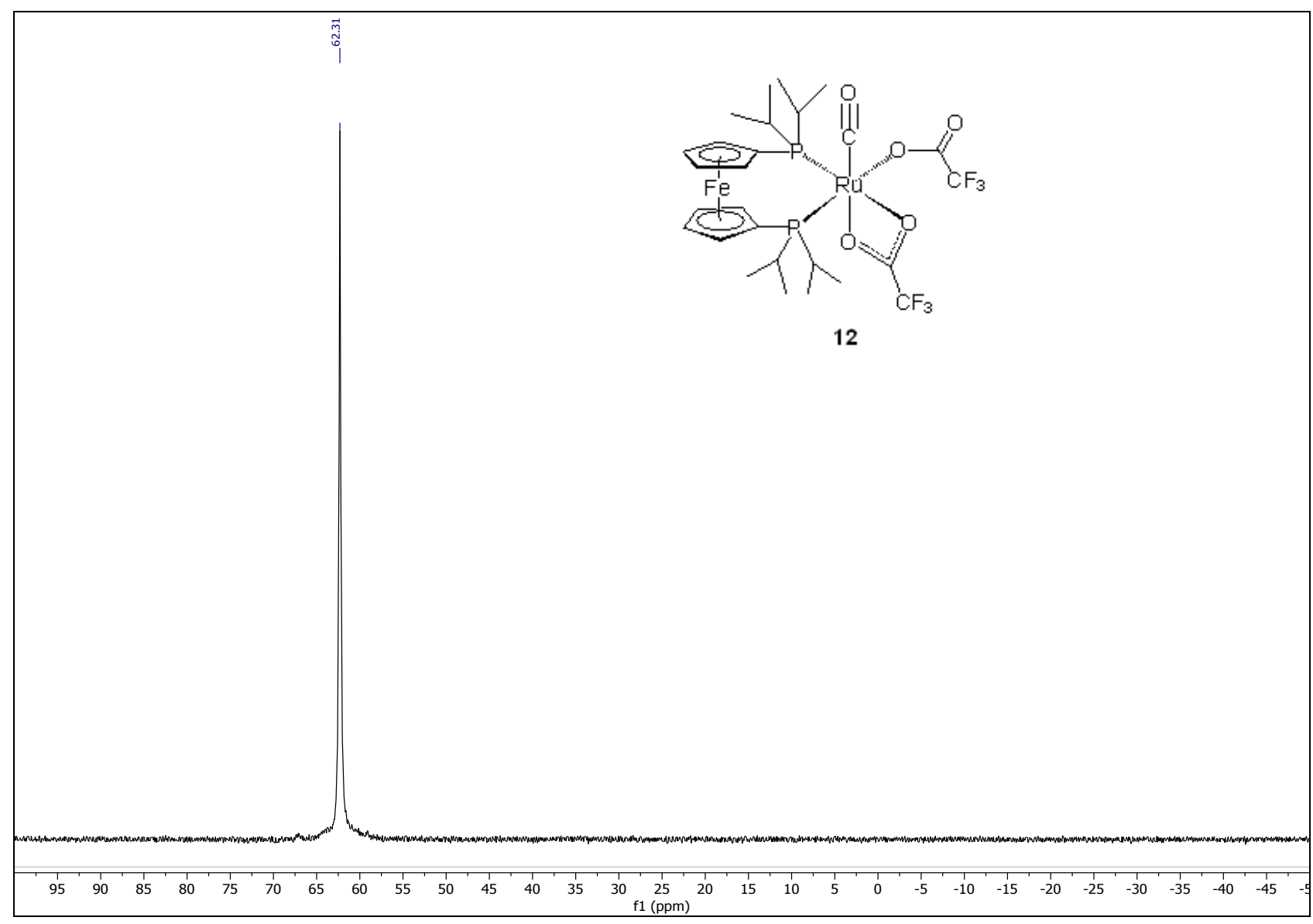

Figure S40. ${ }^{31} \mathrm{P}\left\{{ }^{1} \mathrm{H}\right\}$ NMR spectrum $(162.0 \mathrm{MHz})$ of $\left[\mathrm{Ru}\left(\eta^{1}-\mathrm{OCOCF}_{3}\right)\left(\eta^{2}-\mathrm{OCOCF}_{3}\right)(\mathrm{D} i \mathrm{PPF})(\mathrm{CO})\right]$ (12) in $\mathrm{CD}_{2} \mathrm{Cl}_{2}$ at $25^{\circ} \mathrm{C}$. 


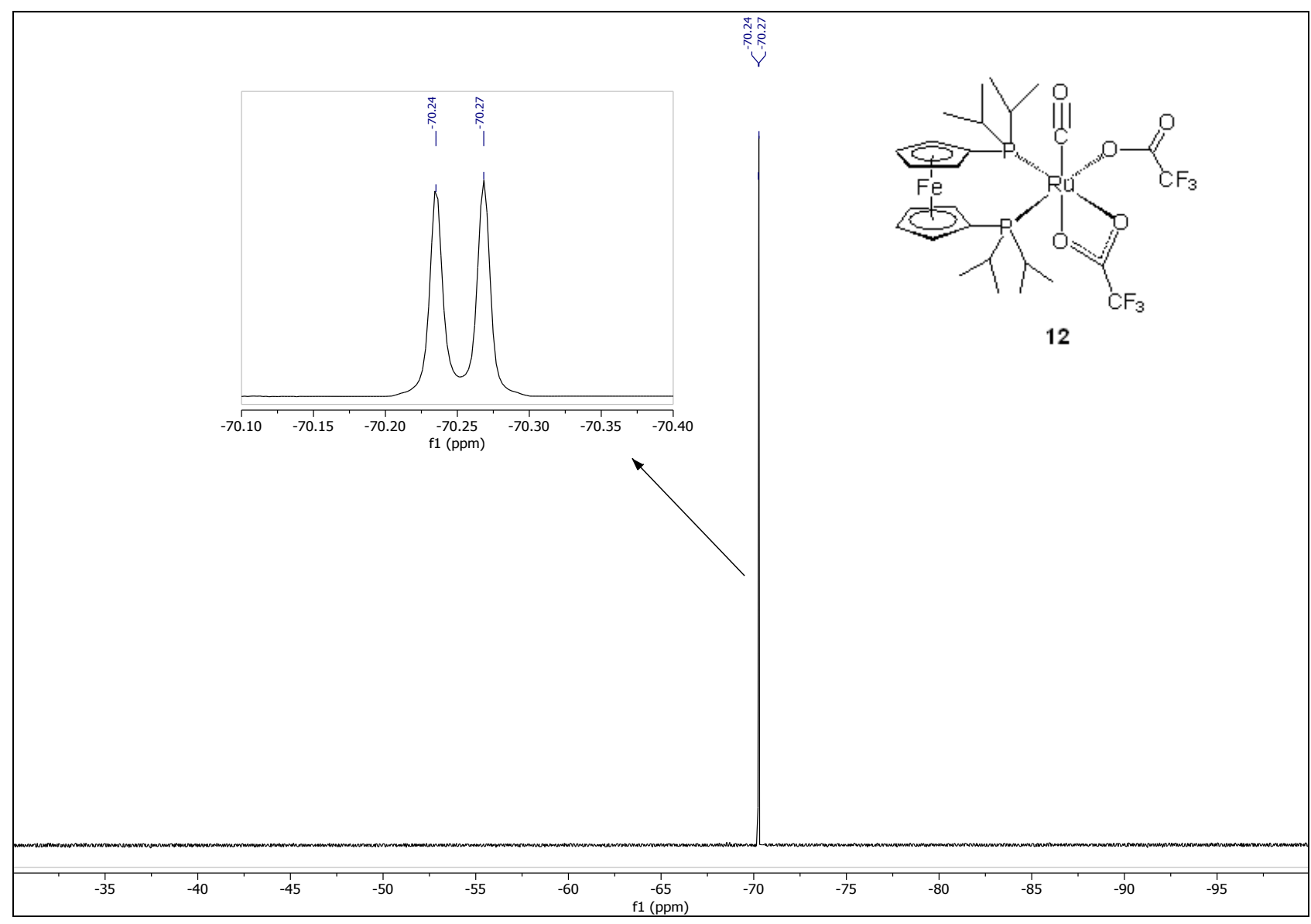

Figure S41. ${ }^{19} \mathrm{~F}\left\{{ }^{1} \mathrm{H}\right\}$ NMR spectrum $(376.6 \mathrm{MHz})$ of $\left[\mathrm{Ru}\left(\eta^{1}-\mathrm{OCOCF}_{3}\right)\left(\eta^{2}-\mathrm{OCOCF}_{3}\right)(\mathrm{D} i \mathrm{PPF})(\mathrm{CO})\right]$ (12) in $\mathrm{CD}_{2} \mathrm{Cl}_{2}$ at $25^{\circ} \mathrm{C}$. 


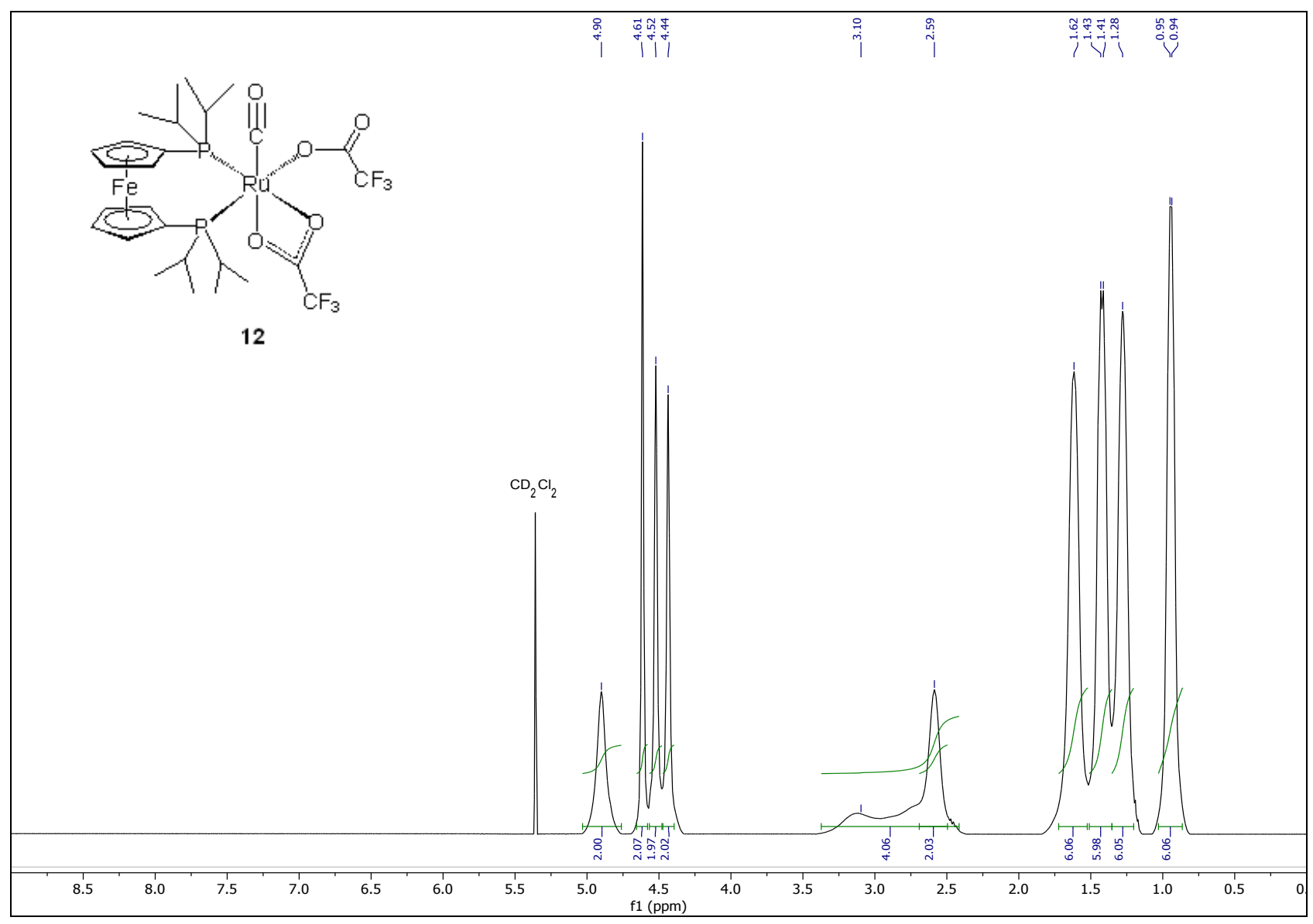

Figure S42. ${ }^{1} \mathrm{H}$ NMR spectrum $(400.1 \mathrm{MHz})$ of $\left[\mathrm{Ru}\left(\eta^{1}-\mathrm{OCOCF}_{3}\right)\left(\eta^{2}-\mathrm{OCOCF}_{3}\right)(\mathrm{D} i \mathrm{PPF})(\mathrm{CO})\right](\mathbf{1 2})$ in $\mathrm{CD}_{2} \mathrm{Cl}_{2}$ at $25^{\circ} \mathrm{C}$. 


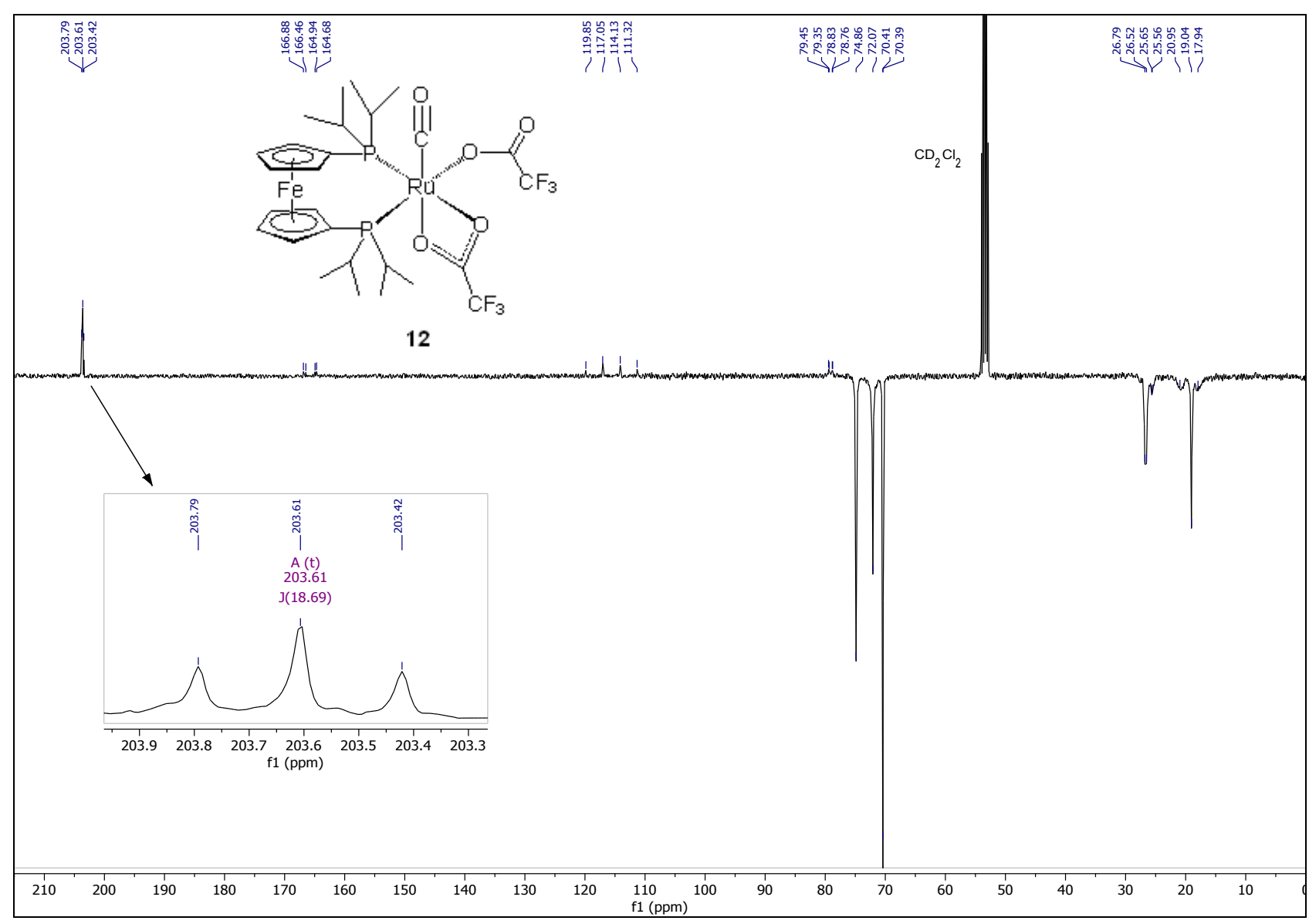

Figure S43. ${ }^{13} \mathrm{C}\left\{{ }^{1} \mathrm{H}\right\}$ DEPTQ NMR spectrum $(100.6 \mathrm{MHz})$ of $\left[\mathrm{Ru}\left(\eta^{1}-\mathrm{OCOCF}_{3}\right)\left(\eta^{2}-\right.\right.$ $\left.\left.\mathrm{OCOCF}_{3}\right)(\mathrm{D} i \mathrm{PPF})(\mathrm{CO})\right](\mathbf{1 2})$ in $\mathrm{CD}_{2} \mathrm{Cl}_{2}$ at $25^{\circ} \mathrm{C}$. 


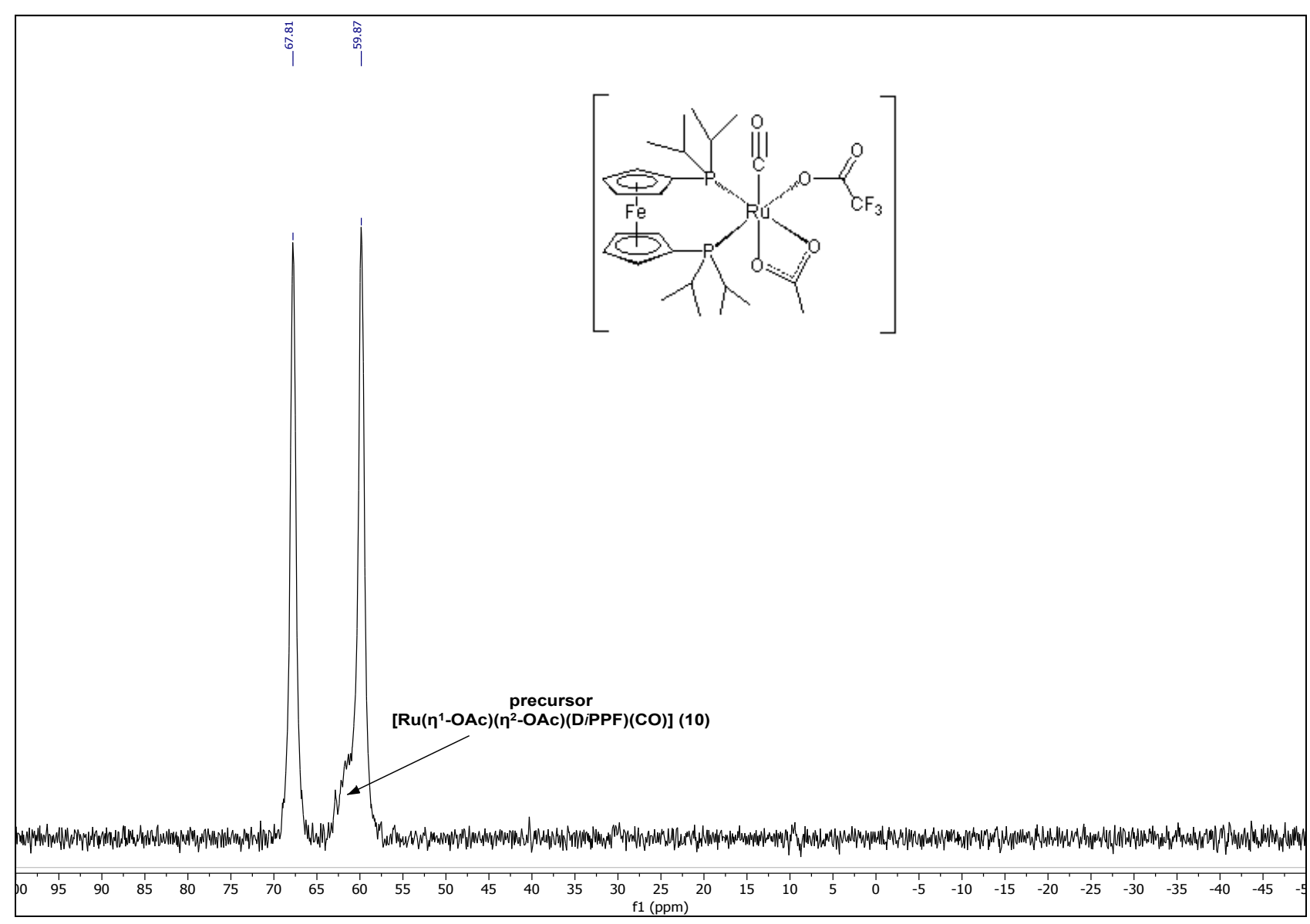

Figure S44. Evidences of the formation of mixed species $\left[\mathrm{Ru}(\mathrm{OAc})\left(\mathrm{OCOCF}_{3}\right)(\mathrm{D} i \mathrm{PPF})(\mathrm{CO})\right]$ by reaction of complex 10 with TFA (1 equiv) in the ${ }^{31} \mathrm{P}\left\{{ }^{1} \mathrm{H}\right\} \mathrm{NMR}$ spectrum $(81.0 \mathrm{MHz})$ in $\mathrm{CD}_{2} \mathrm{Cl}_{2}$ at $20^{\circ} \mathrm{C}$. 


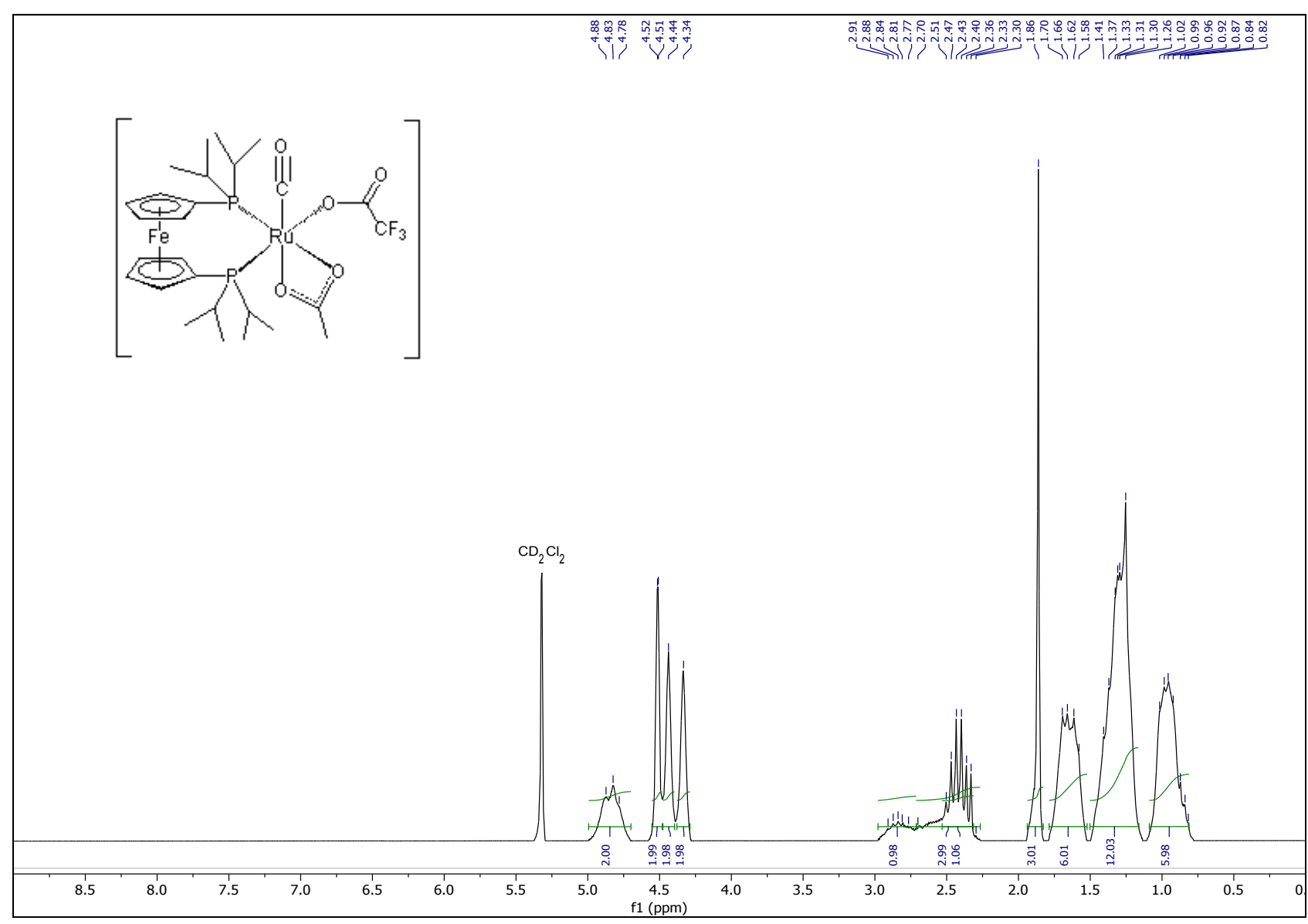

Figure S45. Evidences of the formation of mixed species $\left[\mathrm{Ru}(\mathrm{OAc})\left(\mathrm{OCOCF}_{3}\right)(\mathrm{D} i \mathrm{PPF})(\mathrm{CO})\right]$ by reaction of complex $\mathbf{1 0}$ with TFA (1 equiv) in the ${ }^{1} \mathrm{H}$ NMR spectrum $(200.1 \mathrm{MHz})$ in $\mathrm{CD}_{2} \mathrm{Cl}_{2}$ at 20 ${ }^{\circ} \mathrm{C}$. 


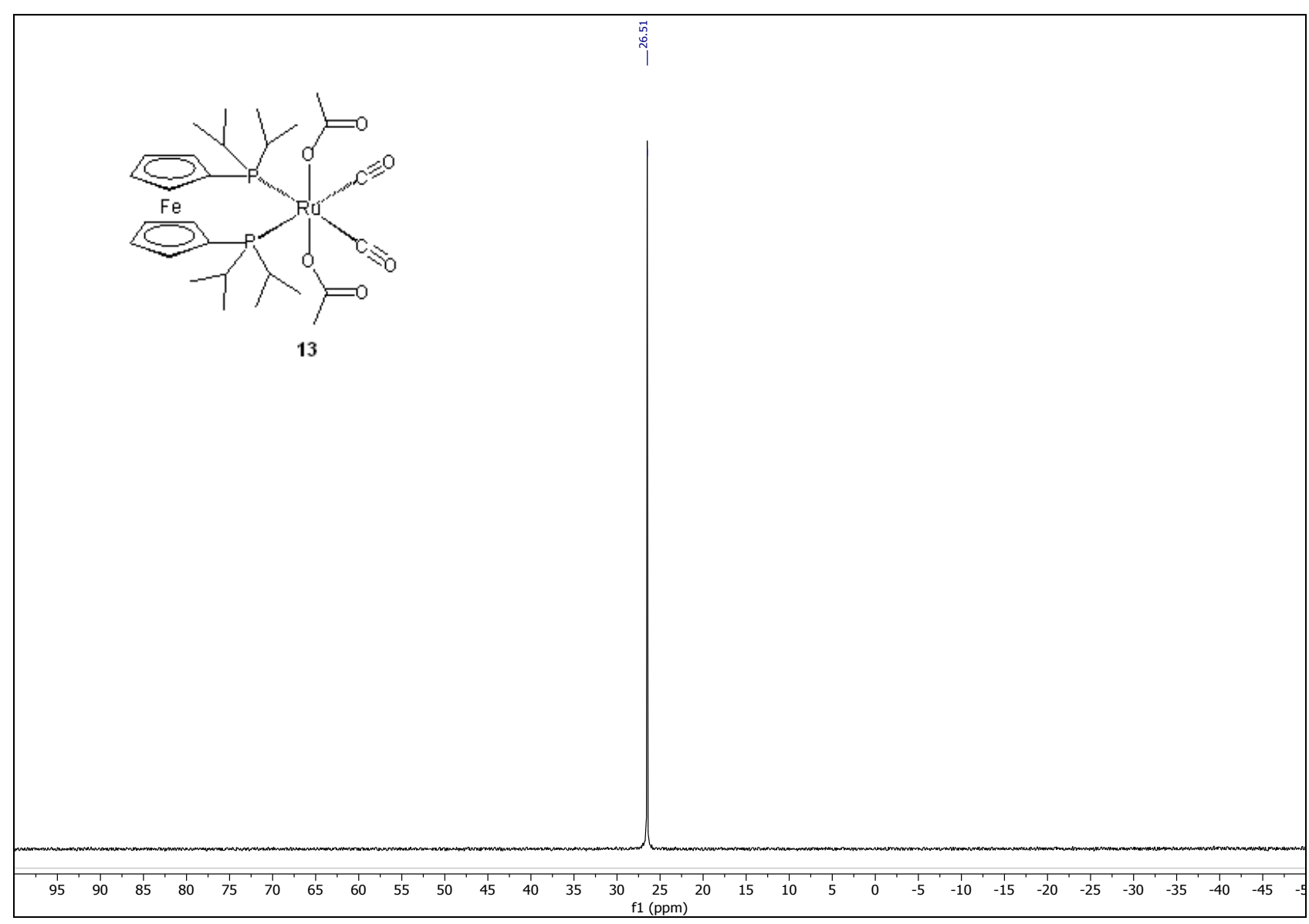

Figure S46. ${ }^{31} \mathrm{P}\left\{{ }^{1} \mathrm{H}\right\}$ NMR spectrum $(81.0 \mathrm{MHz})$ of trans,cis-[Ru( $\left.\left.\eta^{1}-\mathrm{OAc}\right)_{2}(\mathrm{D} i \mathrm{PPF})(\mathrm{CO})_{2}\right](\mathbf{1 3})$ in $\mathrm{CDCl}_{3}$ at $20^{\circ} \mathrm{C}$. 


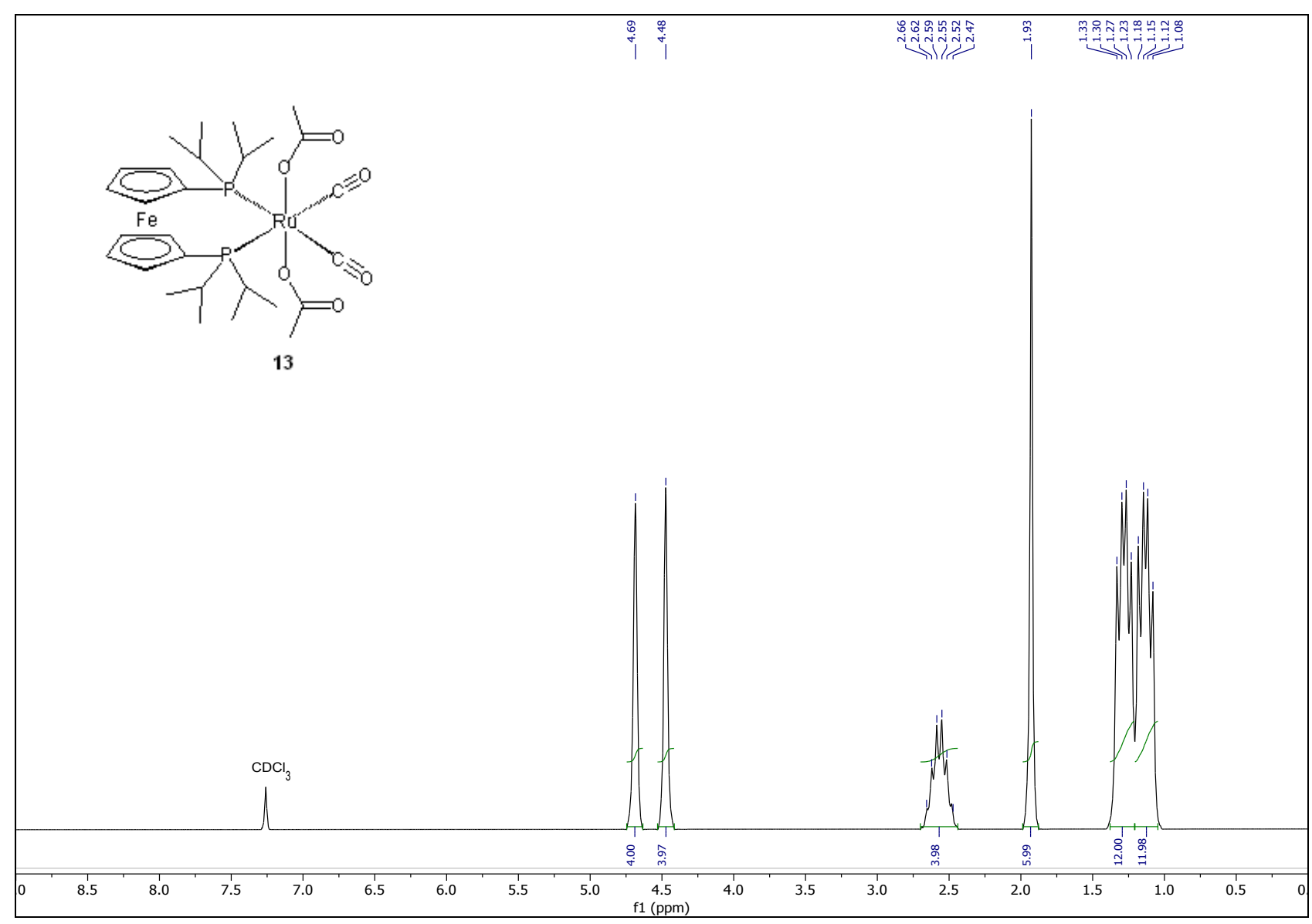

Figure S47. ${ }^{1} \mathrm{H}$ NMR spectrum $(200.1 \mathrm{MHz})$ of trans, cis- $\left[\mathrm{Ru}\left(\eta^{1}-\mathrm{OAc}\right)_{2}(\mathrm{DiPPF})(\mathrm{CO})_{2}\right](\mathbf{1 3})$ in $\mathrm{CDCl}_{3}$ at $20^{\circ} \mathrm{C}$. 


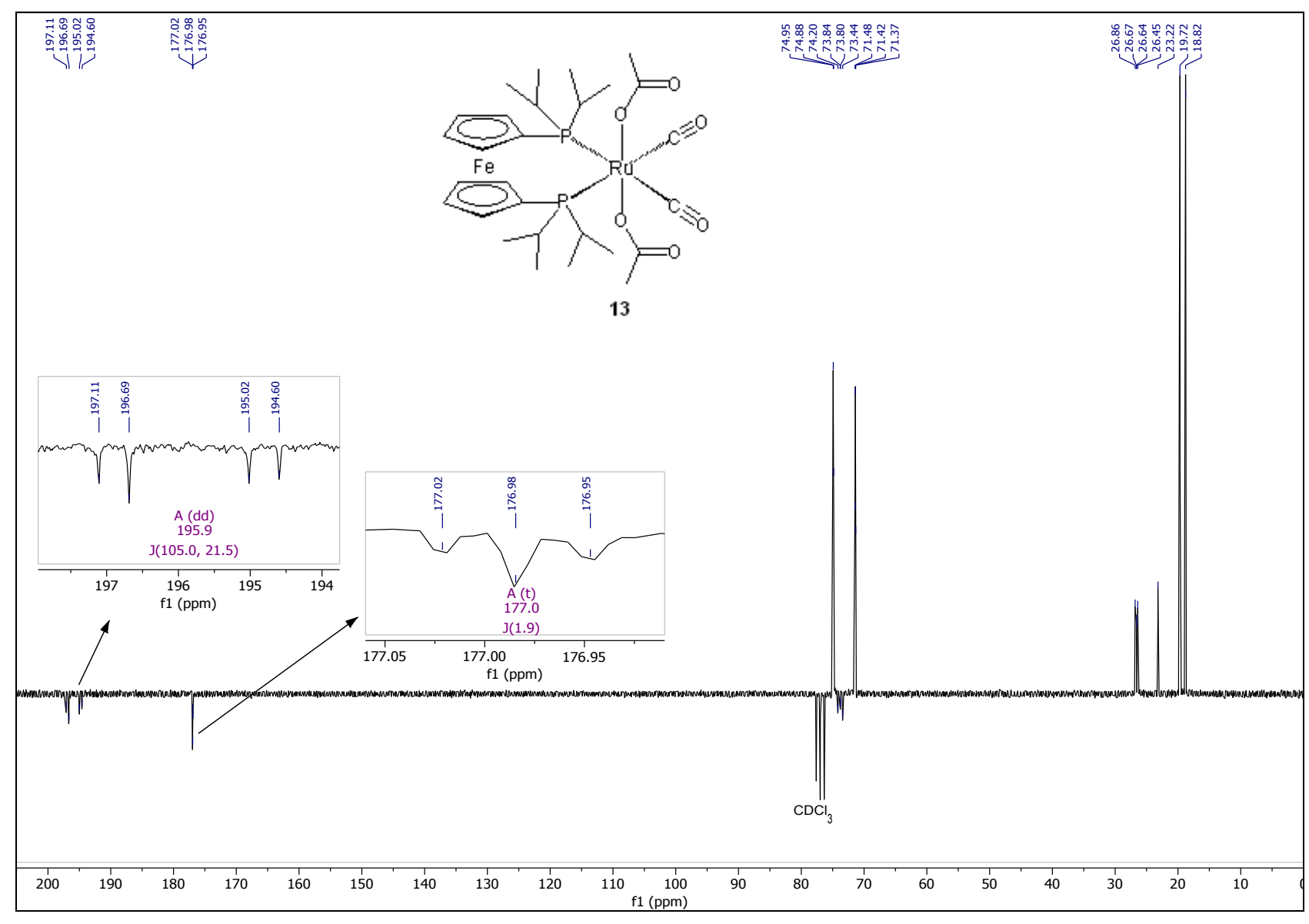

Figure S48. ${ }^{13} \mathrm{C}\left\{{ }^{1} \mathrm{H}\right\} \quad$ PENDANT NMR spectrum $\quad(50.3 \mathrm{MHz})$ of trans,cis-[Ru( $\eta^{1}-$ $\left.\mathrm{OAc})_{2}(\mathrm{D} i \mathrm{PPF})(\mathrm{CO})_{2}\right](\mathbf{1 3})$ in $\mathrm{CDCl}_{3}$ at $20^{\circ} \mathrm{C}$. 


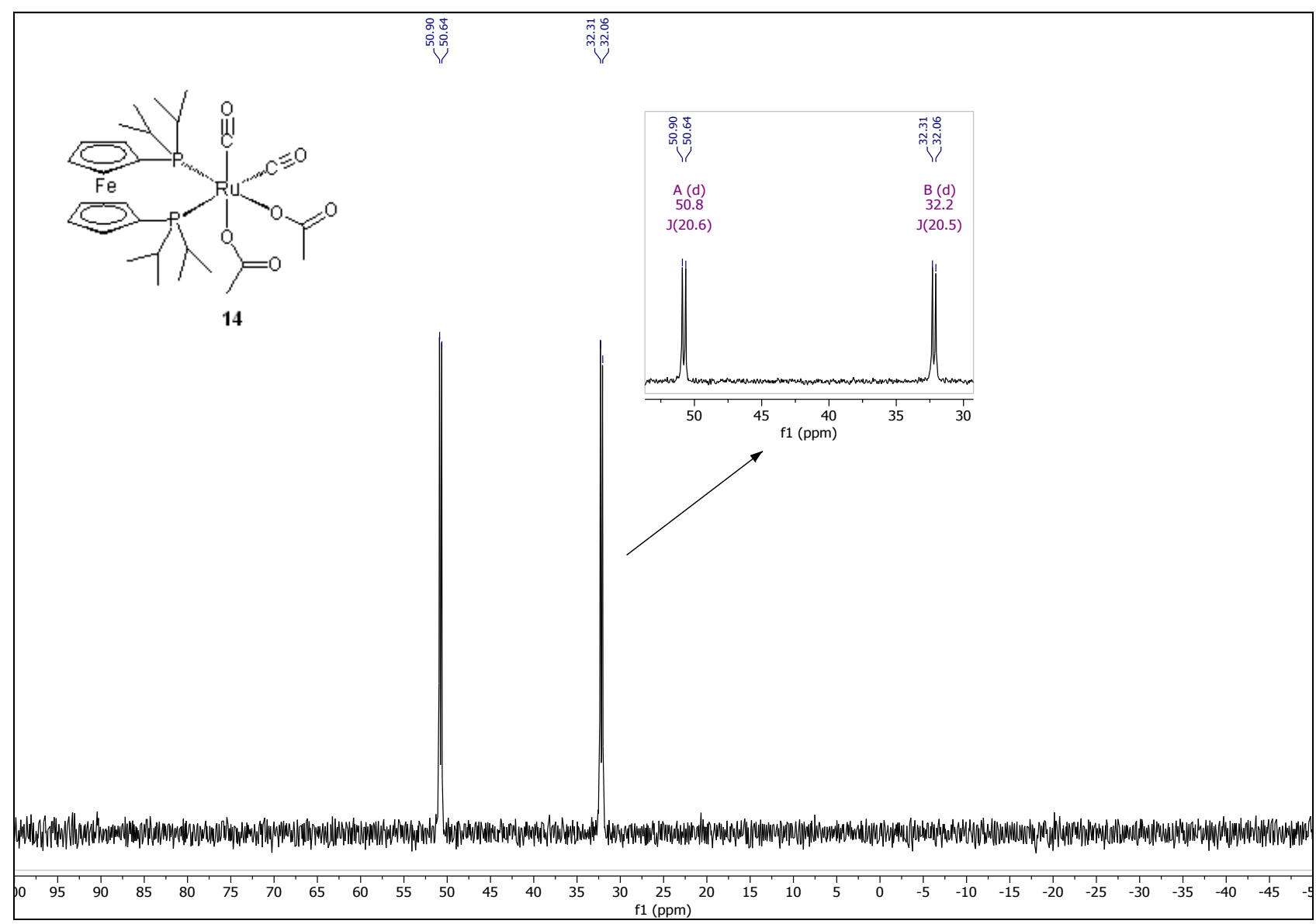

Figure S49. ${ }^{31} \mathrm{P}\left\{{ }^{1} \mathrm{H}\right\}$ NMR spectrum $(81.0 \mathrm{MHz})$ of $c i s, c i s-\left[\mathrm{Ru}\left(\eta^{1}-\mathrm{OAc}\right)_{2}(\mathrm{D} i \mathrm{PPF})(\mathrm{CO})_{2}\right](\mathbf{1 4})$ in $\mathrm{CD}_{2} \mathrm{Cl}_{2}$ at $20{ }^{\circ} \mathrm{C}$. 


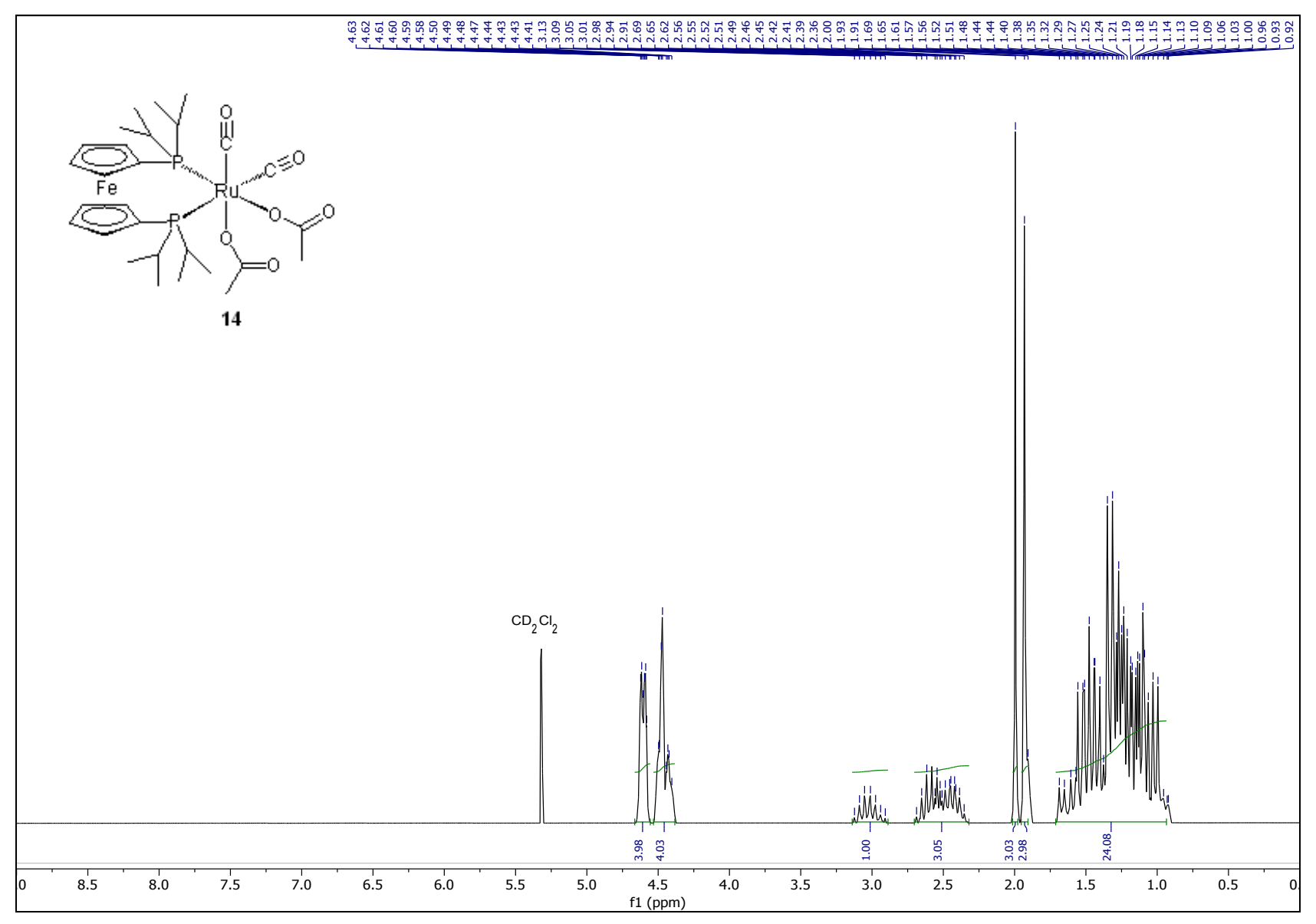

Figure S50. ${ }^{1} \mathrm{H}$ NMR spectrum $(200.1 \mathrm{MHz})$ of $c i s, c i s-\left[\mathrm{Ru}\left(\eta^{1}-\mathrm{OAc}\right)_{2}(\mathrm{D} i \mathrm{PPF})(\mathrm{CO})_{2}\right](\mathbf{1 4})$ in $\mathrm{CD}_{2} \mathrm{Cl}_{2}$ at $20^{\circ} \mathrm{C}$. 


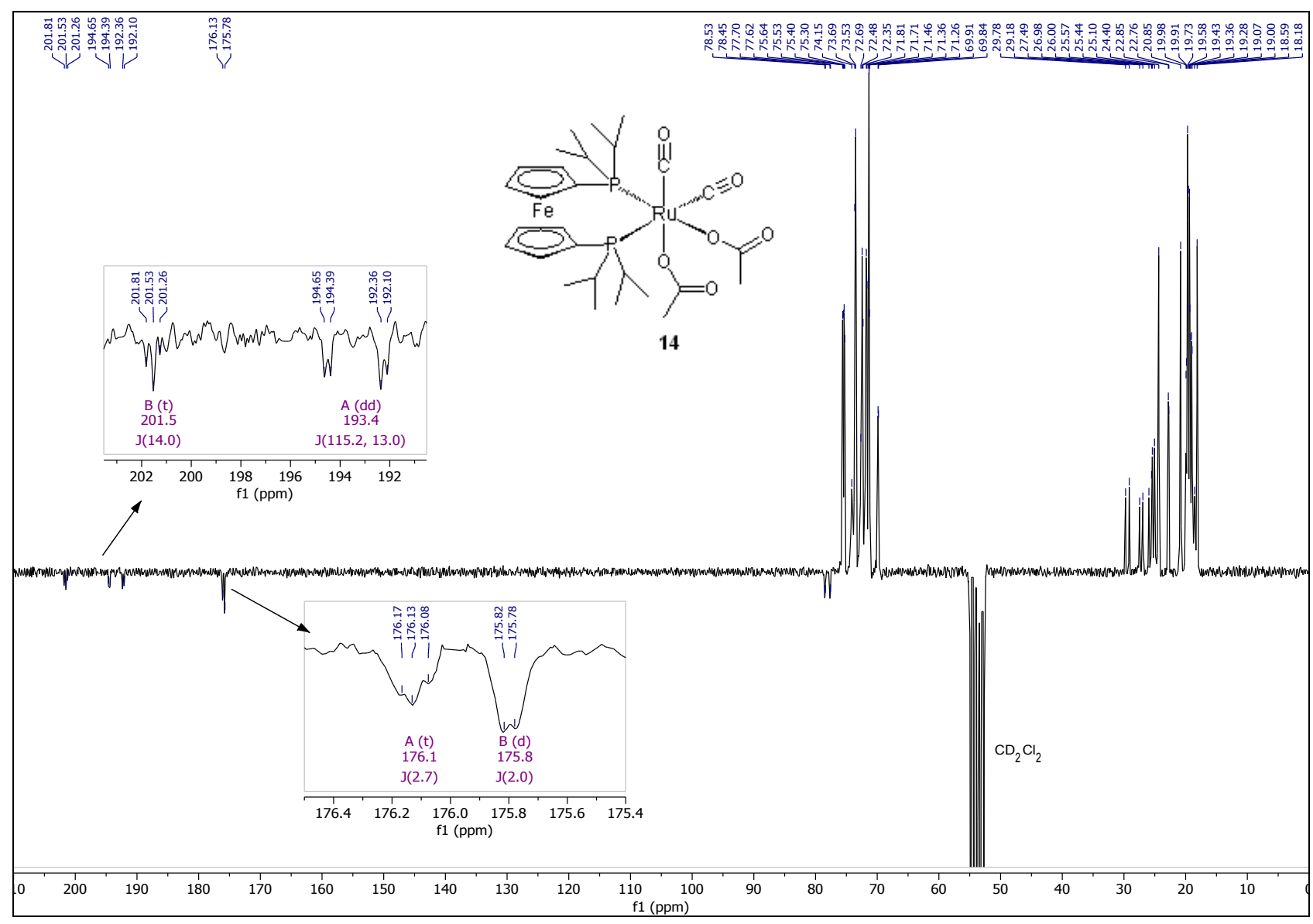

Figure S51. ${ }^{13} \mathrm{C}\left\{{ }^{1} \mathrm{H}\right\}$ PENDANT NMR spectrum $(50.3 \mathrm{MHz})$ of $c i s, c i s-\left[\mathrm{Ru}\left(\eta^{1}-\mathrm{OAc}\right)_{2}(\mathrm{D} i \mathrm{PPF})(\mathrm{CO})_{2}\right]$ (14) in $\mathrm{CD}_{2} \mathrm{Cl}_{2}$ at $20{ }^{\circ} \mathrm{C}$. 


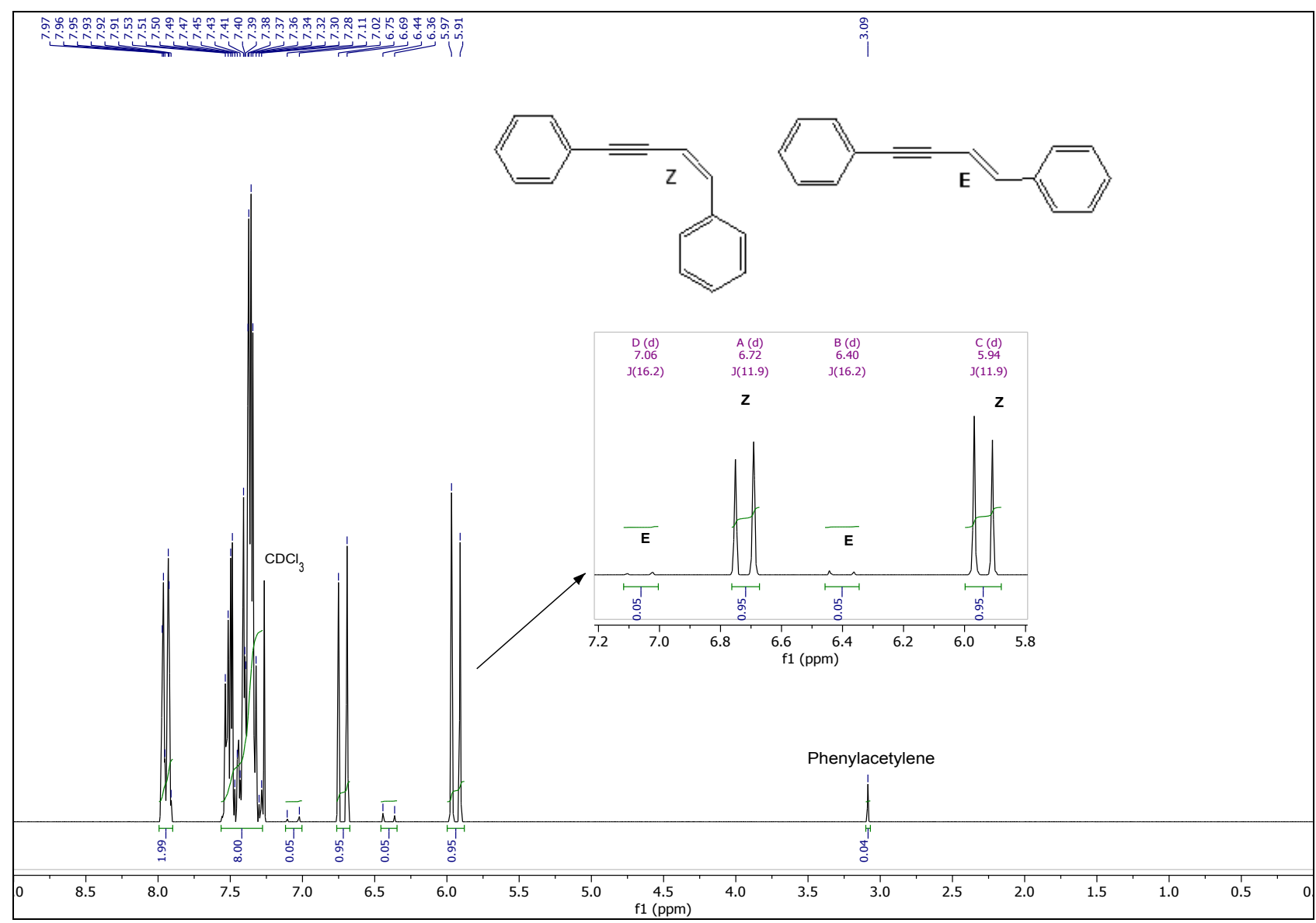

Figure S52. Representative ${ }^{1} \mathrm{H}$ NMR spectrum (200.1 MHz) in $\mathrm{CDCl}_{3}$ at $20{ }^{\circ} \mathrm{C}$ of 1,4-diphenyl-1buten-3-yne obtained from Head-to-Head dimerization of phenylacetylene promoted by complex $\mathbf{1 0}$ at $\mathrm{S} / \mathrm{C}=100(Z / E$ ratio $95 / 5)$.

${ }^{1} \mathrm{H}$ NMR $\left(200.1 \mathrm{MHz}, \mathrm{CDCl}_{3}, 20{ }^{\circ} \mathrm{C}\right): \delta$ 7.99-7.90 (m, 2H; aromatic protons), 7.55-7.46 (m, 2H; aromatic protons), 7.46-7.28 (m, 6H; aromatic protons), $7.06\left(\mathrm{~d},{ }^{3} \mathrm{~J}(\mathrm{H}, \mathrm{H})=16.2 \mathrm{~Hz}, 1 \mathrm{H}\right.$; $\mathrm{Ph}-\mathrm{CH}=\mathrm{CH}$ (E)-isomer), $6.72\left(\mathrm{~d},{ }^{3} J(\mathrm{H}, \mathrm{H})=11.9 \mathrm{~Hz}, 1 \mathrm{H}\right.$; $\mathrm{Ph}-\mathrm{CH}=\mathrm{CH}(Z)$-isomer $), 6.40\left(\mathrm{~d},{ }^{3} J(\mathrm{H}, \mathrm{H})=16.2 \mathrm{~Hz}\right.$, $1 \mathrm{H} ; \mathrm{CH}=\mathrm{CH}$-C $\equiv \mathrm{C}(E)$-isomer $), 5.94\left(\mathrm{~d},{ }^{3} J(\mathrm{H}, \mathrm{H})=11.9 \mathrm{~Hz}, 1 \mathrm{H} ; \mathrm{CH}=\mathrm{CH}-\mathrm{C} \equiv \mathrm{C}(Z)\right.$-isomer $)$. 


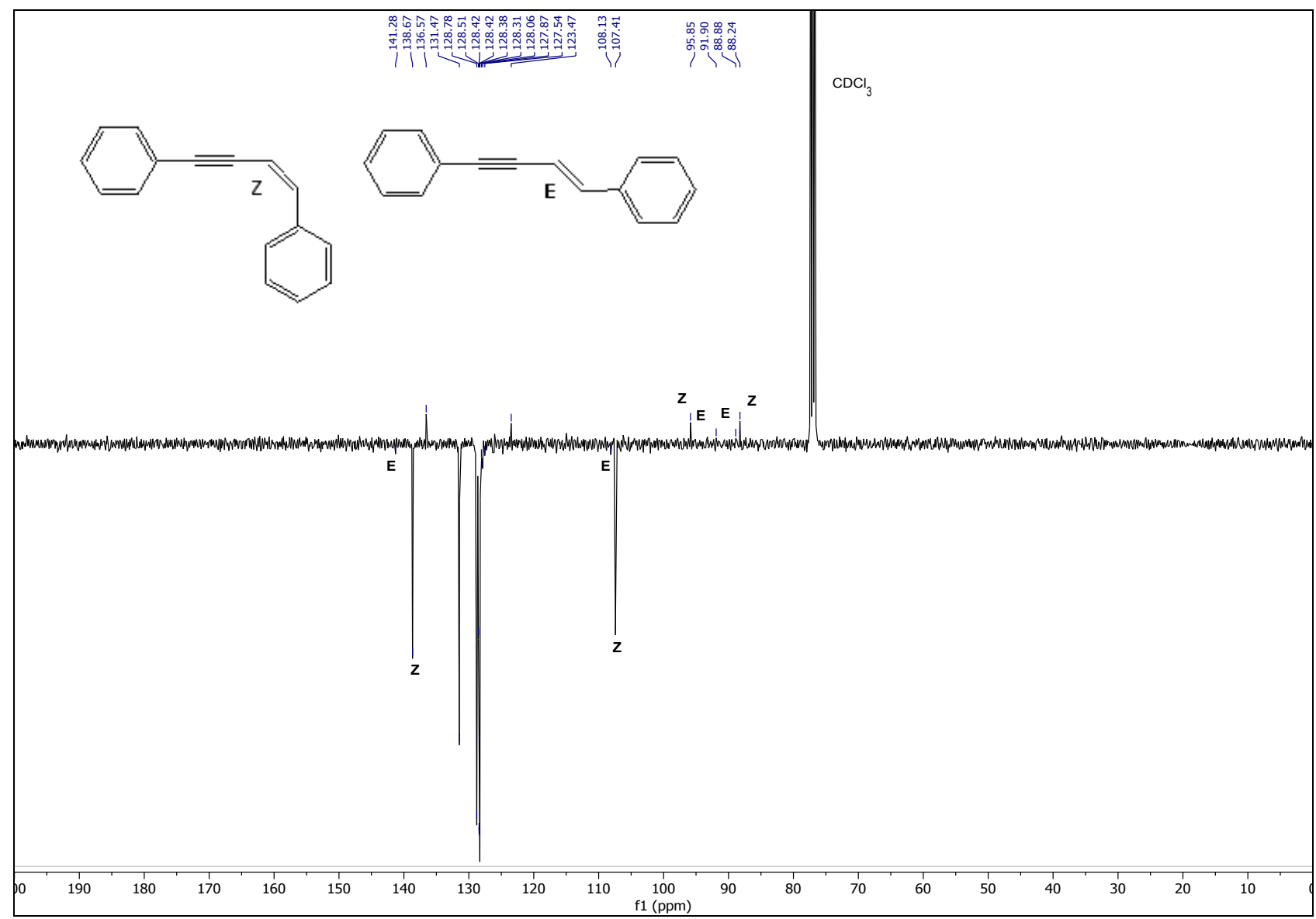

Figure S53. Representative ${ }^{13} \mathrm{C}\left\{{ }^{1} \mathrm{H}\right\}$ DEPTQ NMR spectrum $(100.6 \mathrm{MHz})$ in $\mathrm{CDCl}_{3}$ at $25{ }^{\circ} \mathrm{C}$ of 1,4 diphenyl-1-buten-3-yne obtained from Head-to-Head dimerization of phenylacetylene promoted by complex 10 at $\mathrm{S} / \mathrm{C}=100(Z / E$ ratio $95 / 5)$.

${ }^{13} \mathrm{C}\left\{{ }^{1} \mathrm{H}\right\} \mathrm{NMR}\left(100.6 \mathrm{MHz}, \mathrm{CDCl}_{3}, 25^{\circ} \mathrm{C}\right): \delta 141.3$ (s; $\mathrm{Ph}-\mathrm{CH}=\mathrm{CH}(E)$-isomer), 138.7 (s; $\mathrm{Ph}-\mathrm{CH}=\mathrm{CH}$ (Z)-isomer), 136.6 (s; ipso aromatic carbon (Z)-isomer), 131.5-127.5 (m; aromatic carbon atoms), 123.5 (s; ipso aromatic carbon $(Z)$-isomer), 108.1 (s; $\mathrm{CH}=\mathrm{CH}-\mathrm{C} \equiv \mathrm{C}(E)$-isomer), 107.4 (s; $\mathrm{CH}=C H-$ $\mathrm{C} \equiv \mathrm{C}$ (Z)-isomer), 95.9 (s; $\mathrm{C} \equiv C-\mathrm{CH}_{2} \mathrm{Ph}$ (Z)-isomer), 91.9 (s; $\mathrm{C} \equiv C-\mathrm{CH}_{2} \mathrm{Ph}(E)$-isomer), 88.9 (s; $\mathrm{CH}=\mathrm{CH}-\mathrm{C} \equiv \mathrm{C}(E)$-isomer), $88.2(\mathrm{~s} ; \mathrm{CH}=\mathrm{CH}-C \equiv \mathrm{C}(Z)$-isomer $)$. 


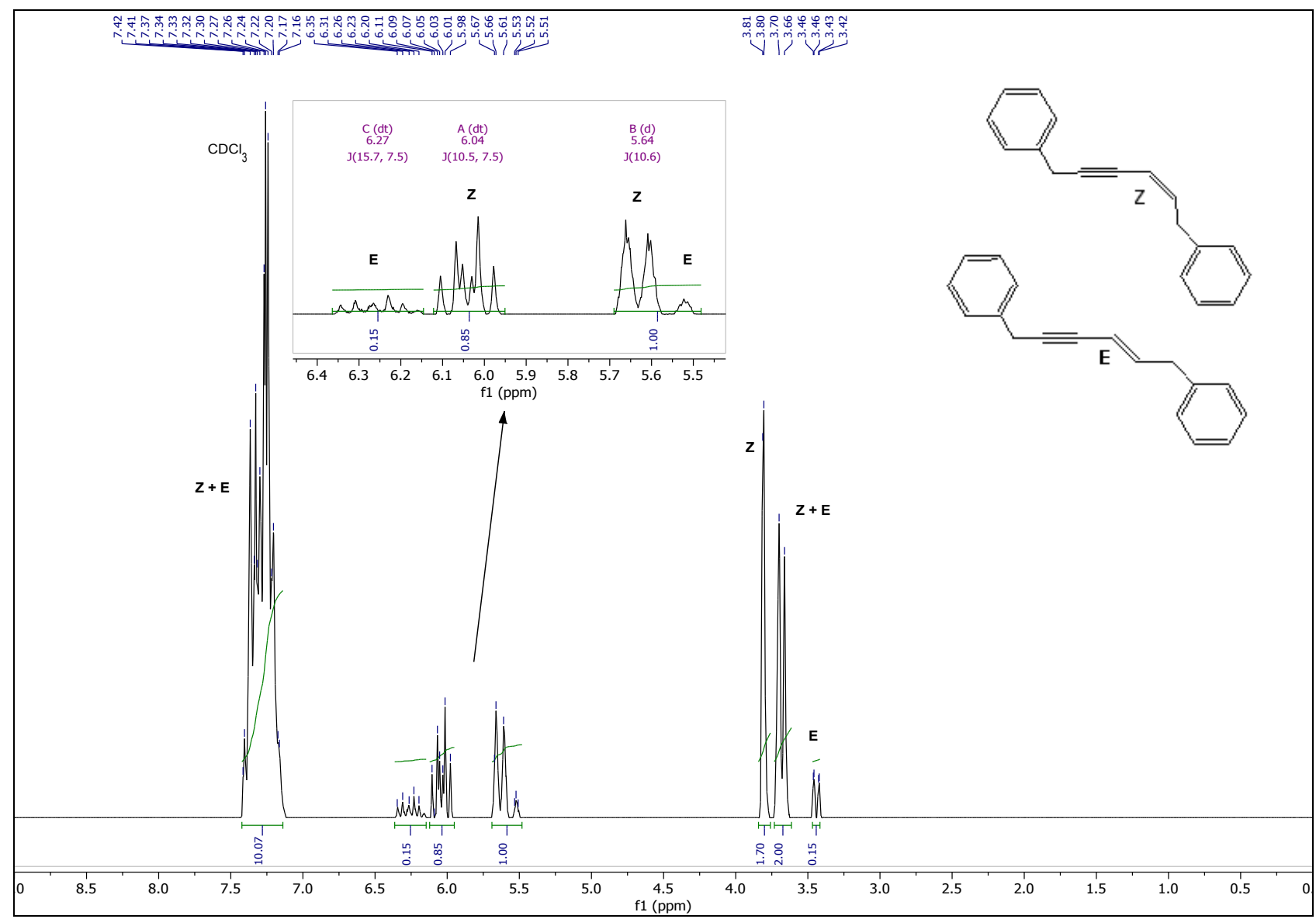

Figure S54. Representative ${ }^{1} \mathrm{H}$ NMR spectrum (200.1 MHz) in $\mathrm{CDCl}_{3}$ at $20{ }^{\circ} \mathrm{C}$ of 1,6-diphenyl-2hexen-4-yne obtained from Head-to-Head dimerization of 3-Phenyl-1-Propyne promoted by complex 10 at $\mathrm{S} / \mathrm{C}=100(Z / E$ ratio $85 / 15)$.

${ }^{1} \mathrm{H}$ NMR $\left(200.1 \mathrm{MHz}, \mathrm{CDCl}_{3}, 20{ }^{\circ} \mathrm{C}\right): \delta 7.43-7.12\left(\mathrm{~m}, 10 \mathrm{H}\right.$; aromatic protons), $6.27\left(\mathrm{dt},{ }^{3} J(\mathrm{H}, \mathrm{H})=\right.$ $15.7 \mathrm{~Hz},{ }^{3} J(\mathrm{H}, \mathrm{H})=7.5 \mathrm{~Hz}, 1 \mathrm{H}$; $\mathrm{Ph}-\mathrm{CH}_{2}-\mathrm{CH}=\mathrm{CH}(E)$-isomer $), 6.04\left(\mathrm{dt},{ }^{3} J(\mathrm{H}, \mathrm{H})=10.5 \mathrm{~Hz},{ }^{3} J(\mathrm{H}, \mathrm{H})=\right.$ $7.5 \mathrm{~Hz}, 1 \mathrm{H} ; \mathrm{Ph}-\mathrm{CH}_{2}-\mathrm{CH}=\mathrm{CH}(\mathrm{Z})$-isomer), $5.64\left(\mathrm{~d},{ }^{3} \mathrm{~J}(\mathrm{H}, \mathrm{H})=10.5 \mathrm{~Hz}, 1 \mathrm{H} ; \mathrm{CH}=\mathrm{CH}-\mathrm{C} \equiv \mathrm{C}(\mathrm{Z})\right.$-isomer). $5.54\left(\mathrm{~d},{ }^{3} J(\mathrm{H}, \mathrm{H})=15.7 \mathrm{~Hz}, 1 \mathrm{H} ; \mathrm{CH}=\mathrm{CH}-\mathrm{C} \equiv \mathrm{C}(E)\right.$-isomer), $3.81\left(\mathrm{~s}, 2 \mathrm{H} ; \mathrm{Ph}-\mathrm{CH}_{2}-\mathrm{CH}=\mathrm{CH}(Z)\right.$-isomer), $3.72\left(\mathrm{~s}, 2 \mathrm{H}\right.$; $\mathrm{Ph}-\mathrm{CH}_{2}-\mathrm{CH}=\mathrm{CH}(E)$-isomer), $3.68\left(\mathrm{~d},{ }^{3} J(\mathrm{H}, \mathrm{H})=7.5 \mathrm{~Hz}, 2 \mathrm{H} ; \mathrm{C} \equiv \mathrm{C}-\mathrm{CH}_{2} \mathrm{Ph}(Z)\right.$-isomer), $3.44\left(\mathrm{~d},{ }^{3} \mathrm{~J}(\mathrm{H}, \mathrm{H})=7.5 \mathrm{~Hz}, 2 \mathrm{H} ; \mathrm{C} \equiv \mathrm{C}-\mathrm{CH}_{2} \mathrm{Ph}(E)\right.$-isomer $)$. 


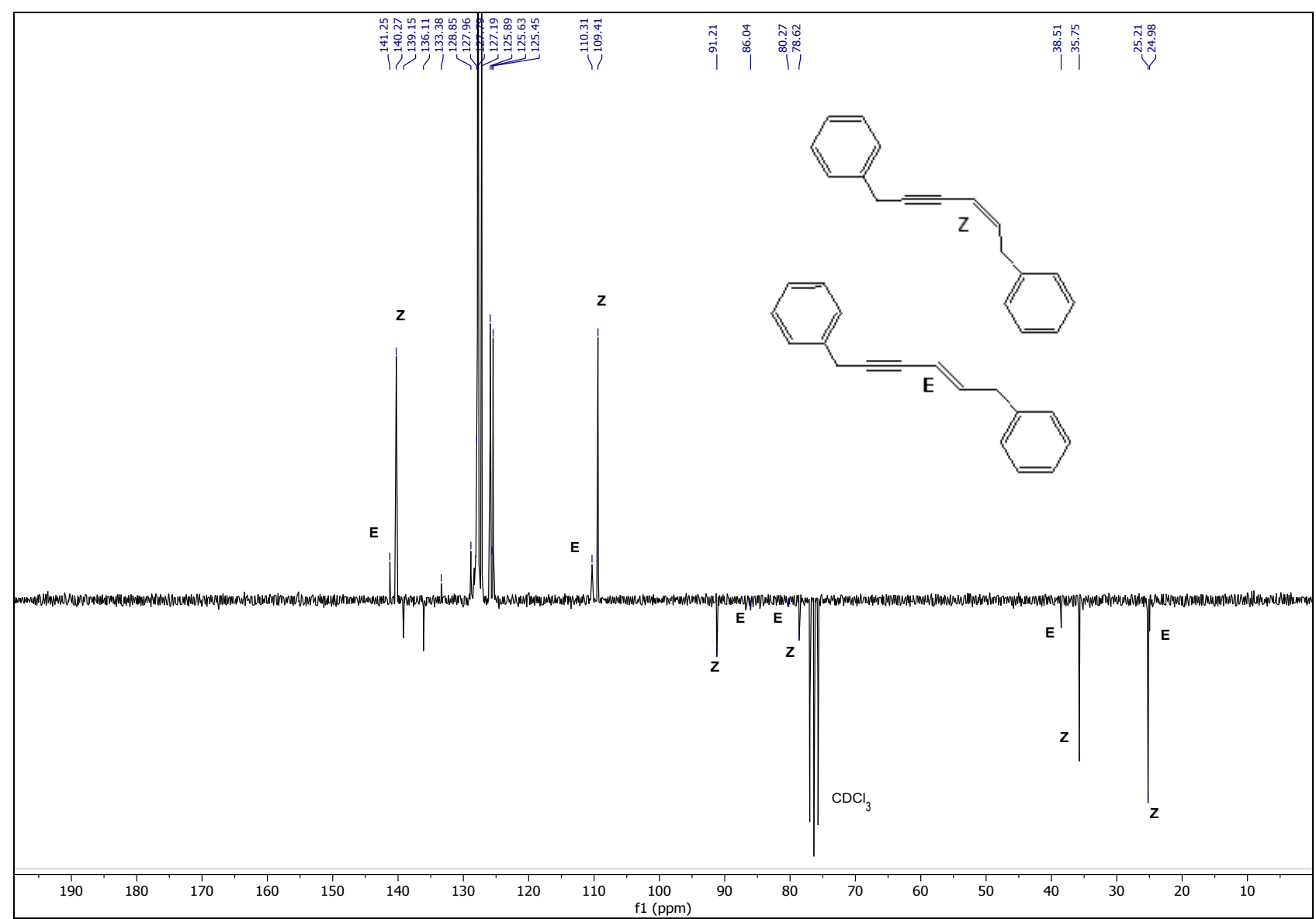

Figure S55. Representative ${ }^{13} \mathrm{C}\left\{{ }^{1} \mathrm{H}\right\}$ PENDANT NMR spectrum $(50.3 \mathrm{MHz})$ in $\mathrm{CDCl}_{3}$ at $20{ }^{\circ} \mathrm{C}$ of 1,6-diphenyl-2-hexen-4-yne obtained from Head-to-Head dimerization of 3-Phenyl-1-Propyne promoted by complex $\mathbf{1 0}$ at $\mathrm{S} / \mathrm{C}=100(Z / E$ ratio $85 / 15)$.

${ }^{13} \mathrm{C}\left\{{ }^{1} \mathrm{H}\right\}$ NMR $\left(50.3 \mathrm{MHz}, \mathrm{CDCl}_{3}, 20{ }^{\circ} \mathrm{C}\right): \delta 141.2\left(\mathrm{~s} ; \mathrm{Ph}-\mathrm{CH}_{2}-\mathrm{CH}=\mathrm{CH}(E)\right.$-isomer), $140.3(\mathrm{~s} ; \mathrm{Ph}-$ $\mathrm{CH}_{2}-\mathrm{CH}=\mathrm{CH}(\mathrm{Z}$ )-isomer), 139.1 (s; ipso aromatic carbon (Z)-isomer), 136.1 (s; ipso aromatic carbon (Z)-isomer), 129.0-124.5 (m; aromatic carbon atoms), 110.3 (s; $\mathrm{CH}=C \mathrm{H}-\mathrm{C} \equiv \mathrm{C}(E)$-isomer), 109.4 (s; $\mathrm{CH}=\mathrm{CH}-\mathrm{C} \equiv \mathrm{C}(\mathrm{Z})$-isomer), 91.2 (s; $\mathrm{C} \equiv C$ - $\mathrm{CH}_{2} \mathrm{Ph}(Z)$-isomer), 86.0 (s; $\mathrm{C} \equiv C$ - $\mathrm{CH}_{2} \mathrm{Ph}(E)$-isomer), 80.3 (s; $\mathrm{CH}=\mathrm{CH}-\mathrm{C} \equiv \mathrm{C}(E)$-isomer), $78.6\left(\mathrm{~s} ; \mathrm{CH}=\mathrm{CH}-\mathrm{C} \equiv \mathrm{C}(Z)\right.$-isomer), 38.5 (s; $\mathrm{Ph}-\mathrm{CH}_{2}-\mathrm{CH}=\mathrm{CH}(E)-$ isomer), 35.7 (s; $\mathrm{Ph}-\mathrm{CH}_{2}-\mathrm{CH}=\mathrm{CH}(\mathrm{Z})$-isomer), 25.2 (s; $\mathrm{C} \equiv \mathrm{C}-\mathrm{CH}_{2} \mathrm{Ph}(\mathrm{Z})$-isomer), 25.0 (s; $\mathrm{C} \equiv \mathrm{C}-\mathrm{CH}_{2} \mathrm{Ph}$ (E)-isomer). 


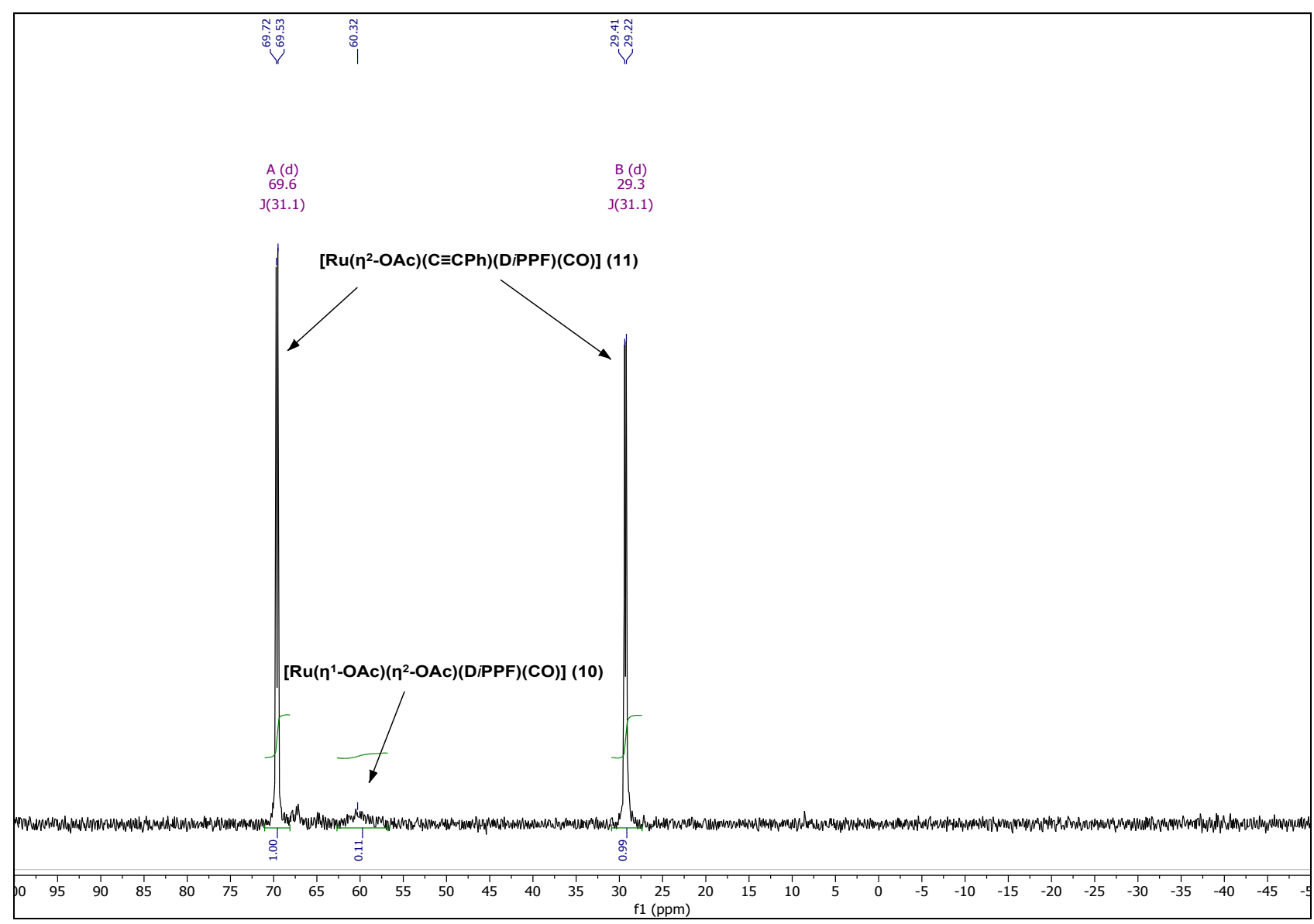

Figure S56. ${ }^{31} \mathrm{P}\left\{{ }^{1} \mathrm{H}\right\}$ NMR spectrum $(81.0 \mathrm{MHz})$ of unpurified reaction mixture of dimerization of phenylacetylene after $40 \mathrm{~h}$ heating at reflux promoted by complex 10 at $\mathrm{S} / \mathrm{C}=30$ in toluene- $d^{8}$. 


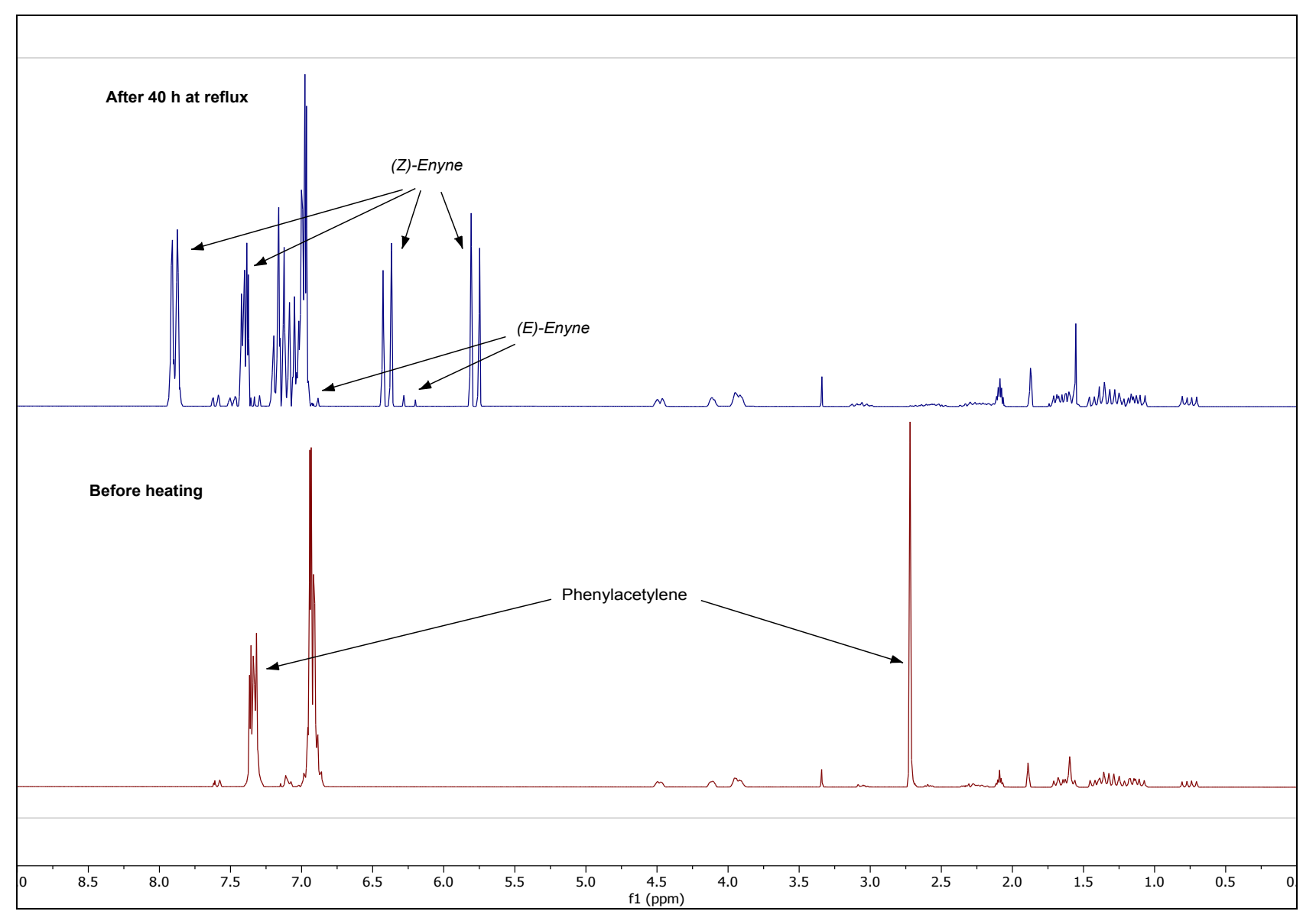

Figure S57. Stacked ${ }^{1}$ H NMR spectrum (200.1 MHz) of unpurified reaction mixture of dimerization of phenylacetylene before the heating and after $40 \mathrm{~h}$ at reflux, promoted by complex $\mathbf{1 0}$ at $\mathrm{S} / \mathrm{C}=30$ in toluene- $d^{8}$. 
Single Crystal X-Ray Structure Determination of Compound 11 (CCDC1989777).

\section{General Data}

Crystals data were collected at $170 \mathrm{~K}$ on an Oxford Diffraction Excalibur diffractometer using graphite monochromated Mo-K radiation equipped with an Eos CCD detector $(\lambda=0.71073 \AA)$. The structure was solved by direct methods with the aid of successive difference Fourier maps, and were refined against all data using SHELXL-2014 in conjunction with SHELXLE, ${ }^{1}$ and refined by full matrix least-squares minimizing $\Sigma w\left(\mathrm{~F}_{\mathrm{o}}{ }^{2}-\mathrm{F}_{\mathrm{c}}{ }^{2}\right)^{2}$ with the SHELXL-2014 weighting scheme. ${ }^{2}$ Direct methods determined the positions of all the atoms. All programs were used within the Olex suite. ${ }^{3}$ All non-hydrogen atoms were refined anisotropically. Structural illustration were drawn with ORTEP-3 for Windows. ${ }^{4}$ The measurements are performed on a single crystal coated with perfluorinated ether. The crystal is fixed on the top of a glass fiber and transferred to the diffractometer. The crystal is frozen under a stream of cold nitrogen. CCDC 1989777 contains the supplementary crystallographic data for this paper. These data are provided free of charge by The Cambridge Crystallographic Data Centre via www.ccdc.cam.ac.uk/data_request/cif or via https://www.ccdc.cam.ac.uk/services/structure_deposit/. 


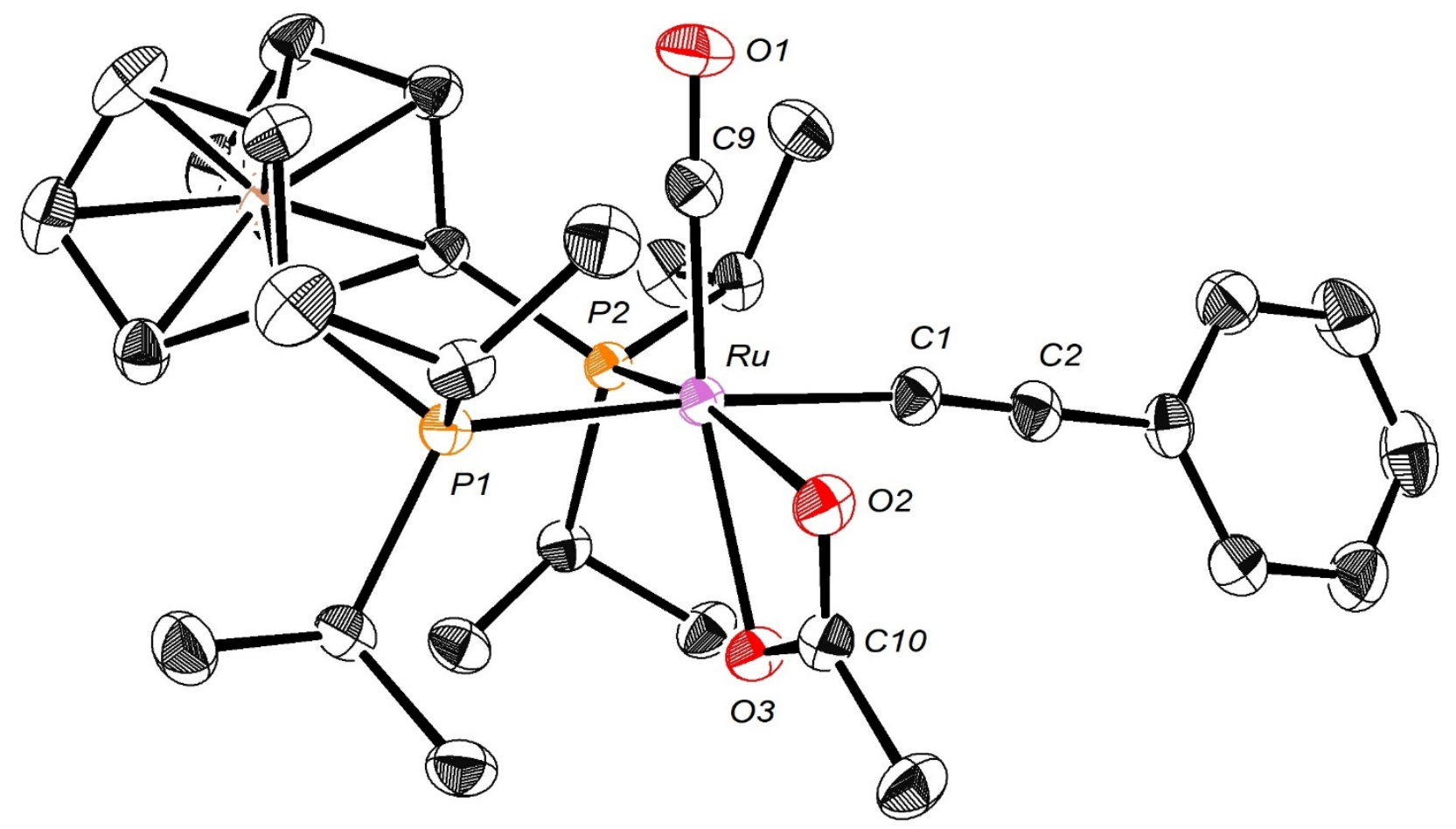

Figure S58. Molecular structure of complex 11 (CCDC 1989777) 
Single Crystal X-Ray Structure Determination of Compound 11 (CCDC 1989777).

\section{Detailed Crystallographic Data.}

\section{Crystal Data:}

Compound

Chemical formula of the crystal

Formula weight $\left(M_{\mathrm{r}}\right)$

Temperature

Wavelength

Radiation type

Crystal size

Crystal system

Space group

Unit cell dimensions

Volume

Z

Absorption coefficient

Data collection and structure refinement:

Diffractometer

Absorption correction

No. of measured, independent

Xcalibur, Eos

Analytical

CrysAlis Pro 1.171.38.46 (Rigaku Oxford Diffraction, 2015) Analytical numeric absorption correction using a multifaceted crystal model based on expressions derived by R.C. Clark \& J.S. Reid. (Clark, R. C. \& Reid, J. S. (1995). Acta Cryst. A51, 887-897) Empirical absorption correction using spherical harmonics, implemented in SCALE3 ABSPACK scaling algorithm

and observed $[I>2 \sigma(I)]$ reflection 83940, 8449, 6809

$\mathrm{R}$ (int)

0.070 
Max. and min. transmission

$(\sin \theta / \lambda)_{\max }\left(\AA^{-1}\right)$

Structure refinement:

$R\left[F^{2}>2 \sigma\left(F^{2}\right)\right], w R\left(F^{2}\right), S$

No. of reflections

No. of parameters

$\mathrm{H}$-atom treatment

$\left.\Delta\rangle_{\max }, \Delta\right\rangle_{\min }\left(\mathrm{e} \AA^{-3}\right)$
0.941 and 0.906

0.649

$0.034,0.076,1.06$

8449

434

$\mathrm{H}$-atom parameters constrained

$0.49,-0.33$ 


\section{References.}

(1) (a) Sheldrick, G., Crystal structure refinement with SHELXL. Acta Cryst. 2015, C71, 3-8. (b) Sheldrick, G., SHELXT - Integrated space-group and crystal-structure determination. Acta Cryst. 2015, $A 71,3-8$.

(2) Hubschle, C. B.; Sheldrick, G. M.; Dittrich, B., ShelXle: a Qt graphical user interface for SHELXL. J. Appl. Cryst. 2011, 44, 1281-1284.

(3) Dolomanov, O. V.; Bourhis, L. J.; Gildea, R. J.; Howard, J. A. K.; Puschmann, H., OLEX2: a complete structure solution, refinement and analysis program. J. Appl. Cryst. 2009, 42, 339-341.

(4) Farrugia, L., ORTEP-3 for Windows - a version of ORTEP-III with a Graphical User Interface (GUI). J. Appl. Crystallogr. 1997, 30, 565-567. 